\title{
ON BREAKDOWN CRITERIA FOR NONVACUUM EINSTEIN EQUATIONS
}

\author{
ARICK SHAO
}

\begin{abstract}
The recent "breakdown criterion" result [17] of S. Klainerman and I. Rodnianski stated roughly that an Einstein-vacuum spacetime, given as a CMC foliation, can be further extended in time if the second fundamental form and the derivative of the lapse of the foliation are uniformly bounded. This theorem and its proof were extended to Einstein-scalar and Einstein-Maxwell spacetimes in the thesis [21]. In this paper, we state the main results of 21, and we summarize and discuss their proofs. In particular, we will discuss the various issues resulting from nontrivial Ricci curvature and the coupling between the Einstein and the field equations.
\end{abstract}

\section{INTRODUCTION}

The general breakdown/continuation problem for PDE is the following:

Under what conditions can an existing local solution of an evolution equation on a finite interval $\left[T_{0}, T\right)$ be further continued past $T$ ?

This can be equivalently posed as the breakdown of such a solution at a finite time implying the violation of such conditions. The determination of such breakdown criteria can be a potentially useful step toward characterizing the blowup of solutions. Furthermore, in some instances, such breakdown conditions can be critical tools for proving global existence results.

In this paper, we will consider this breakdown problem for the Einstein-scalar (E-S) and Einstein-Maxwell (E-M) equations in the CMC gauge. The results and proofs described in this paper are extensions of those in [17, which established the analogous breakdown criterion for the Einstein-vacuum (E-V) case. The matter field present in these nonvacuum cases presents additional issues to be addressed. The full details of this work can be found in the original report [21].

1.1. Classical Results. Consider, as a model example, the initial value problem for the following nonlinear wave equation on $\mathbb{R}^{1+3}$ :

$$
\square \phi=\left(\partial_{t} \phi\right)^{2},\left.\quad \phi\right|_{t=0}=\phi_{0},\left.\partial_{t} \phi\right|_{t=0}=\phi_{1} .
$$

From classical theory, cf. [20, Thm. 22], we have the following results:

- Given initial data $\left(\phi_{0}, \phi_{1}\right) \in Z_{s}=H^{s}\left(\mathbb{R}^{3}\right) \times H^{s-1}\left(\mathbb{R}^{3}\right)$, where $s>5 / 2$, then a unique solution to (11) exists in the space

$$
X_{s, T^{\prime}}=C\left(\left[0, T^{\prime}\right] ; H^{s}\left(\mathbb{R}^{3}\right)\right) \cap C^{1}\left(\left[0, T^{\prime}\right] ; H^{s-1}\left(\mathbb{R}^{3}\right)\right)
$$

for sufficiently small $T^{\prime}>0$ depending on the $Z_{s}$-norm of the initial data.

- The maximal time of existence $T$, i.e., the supremum of such $\tau$ 's for which a solution exists to time $\tau$, depends on the $Z_{s}$-norm of the initial data.

2000 Mathematics Subject Classification. 35Q76 (Primary) 83C05, 83C22, 35L05 (Secondary). 
A continuation result follows naturally as a companion to local well-posedness: Suppose a solution $\phi$ on a finite time interval $[0, T)$ to (11) is in $X_{s, T^{\prime}}$ for every $0<T^{\prime}<T$, where $s>5 / 2$. If $\phi$ also satisfies the criterion

$$
\|\partial \phi\|_{L^{\infty}\left([0, T) \times \mathbb{R}^{3}\right)}<\infty
$$

then $\phi$ can be extended past time $T$ as a solution of (1). Moreover, this extension is an element of $X_{s, T+\epsilon}$ for small $\epsilon>0$.

In terms of breakdown, this can be equivalently stated as follows:

Suppose a solution $\phi$ on a finite time interval $[0, T)$ to (11) is in $X_{s, T^{\prime}}$ for every $0<T^{\prime}<T$, where $s>5 / 2$. Then, if $\phi$ breaks down at time $T$, i.e., if $\phi$ cannot be extended as above, then $\partial \phi \notin L^{\infty}\left([0, T) \times \mathbb{R}^{3}\right)$.

The main idea behind the result is the following observation: if (2) holds, then we can uniformly bound the $H^{s}\left(\mathbb{R}^{3}\right) \times H^{s-1}\left(\mathbb{R}^{3}\right)$-norms of $\left(\phi, \partial_{t} \phi\right)$ on each timeslice $\{\tau\} \times \mathbb{R}^{3}$, where $0<\tau<T$. Therefore, we can apply the previous local wellposedness result to generate local solutions existing for a fixed time $\epsilon>0$ with each of the above cross-sections as the initial data. By uniqueness, we can patch these local solutions into a solution which exists on the interval $[0, T+\epsilon)$.

Remark. The condition (2) is certainly not optimal, since slightly weaker iterated norm conditions are also known to be sufficient.

A multitude of breakdown results have been established for other evolution equations. For example, consider the incompressible 3-dimensional Euler equations

$$
\partial_{t} u+u \cdot \nabla u+\nabla p \equiv 0, \quad \nabla \cdot u \equiv 0,
$$

where $u: \mathbb{R}^{1+3} \rightarrow \mathbb{R}^{3}$ represents the velocity and $p: \mathbb{R}^{1+3} \rightarrow \mathbb{R}$ represents the pressure. In addition, define the vorticity of $u$ to be the curl $\omega=\nabla \times u$ of $u$.

A well-known result of Beale, Kato, and Majda in 3] established that if a local finite-time solution has its vorticity bounded in the $L_{t}^{1} L_{x}^{\infty}$-norm, then the solution can be extended further in time. 1 An important point here is that unlike the condition (2), we need not bound all components of the derivative of the solution. The proof is in principle like that of the nonlinear wave equation; we use this $L_{t}^{1} L_{x}^{\infty}$ bound on the vorticity in order to derive uniform energy bounds related to the local well-posedness theory of the equations (3).

Another example of a breakdown condition lies in the paper [9] of Eardley and Moncrief on the Yang-Mills equation in $\mathbb{R}^{1+3}$. 2 In this setting, a sufficient continuation criterion is an $L^{\infty}$-bound on the Yang-Mills curvature $F$. Using the standard representation formula for the wave equation, however, one can demonstrate that such a uniform bound always holds. The immediate consequence, then, is a global existence result. Furthermore, Chruściel and Shatah, in [8], generalized this result to globally hyperbolic $(1+3)$-dimensional Lorentzian manifolds using mostly the same principles, but applying instead the representation formula of [1].

1.2. Results in General Relativity. In general relativity, a number of breakdown results have been established for the E-V equations. For example, by solving the equations in the standard fashion by imposing favorable gauge conditions, such as wave coordinates, one can show that given a solution in which an $L^{\infty}$-bound

\footnotetext{
${ }^{1}$ The $L_{t}^{1} L_{x}^{\infty}$-norm of $u$ is the $L^{1}$-norm of the function $t \mapsto\|u(t)\|_{L^{\infty}\left(\mathbb{R}^{3}\right)}$.

${ }^{2}$ For simplicity, we neglect the Higgs field, which was also discussed in 9 .
} 
holds for $\partial \mathfrak{G}$, where $\mathfrak{G}$ is the spacetime metric and $\partial$ refers to the gauge coordinate derivatives, then the solution can be further continued. Examples of such results include [6] and, more recently, 2]. Although such a condition is quite analogous to the model case of the nonlinear wave equation, it is also non-geometric, as it depends on the specific choice of coordinates. Moreover, the condition requires bounds on all components of the derivative of the metric.

Another more geometric breakdown result for the $\mathrm{E}-\mathrm{V}$ equations was given by $\mathrm{M}$. Anderson in [1]. Here, the continuation criterion is an $L^{\infty}$-bound on the curvature of the spacetime. While this is clearly geometric, it does have the added disadvantage of depending on essentially two derivatives of the spacetime metric.

The next point of discussion is the improved breakdown result of S. Klainerman and I. Rodnianski for E-V spacetimes, presented in [17. Their main result, stated in [17, Thm. 1.1], can be summarized as follows:

Theorem 1. Suppose $(M, g)$ is an E-V spacetime, given as a CMC foliation

$$
M=\bigcup_{t_{0}<\tau<t_{1}} \Sigma_{\tau}, \quad t_{0}<t_{1}<0,
$$

where each $\Sigma_{\tau}$ is a compact spacelike hypersurface of $M$ satisfying the constant mean curvature condition $\operatorname{tr} k \equiv \tau$, and where $k$ denotes the second fundamental form of $\Sigma_{\tau}$ in $M$. In addition, let $n$ denote the lapse of the $\Sigma_{\tau}$ 's, and assume the following breakdown criterion holds:

$$
\|k\|_{L^{\infty}(M)}+\|\nabla(\log n)\|_{L^{\infty}(M)}<\infty .
$$

Then, the spacetime $(M, g)$ can be extended as an $E$-V spacetime in the CMC gauge to some time $t_{1}+\epsilon$ for some $\epsilon>0$.

We make note of the following important features of Theorem 1

- This result is more geometric than the previous coordinate breakdown condition, whose statement required the choice of an entire coordinate system. Theorem 1, on the other hand, relies only on the (constant mean curvature) time foliation to state the breakdown criterion.

- Both $k$ and $\nabla(\log n)$ in (4) reside at the level of one derivative of $g$. Also, these quantities do not represent all components of one derivative of $g$.

The proof of Theorem 1 is a complicated affair which in its totality spans several papers: [12, 13, 14, 15, 16, 17, 24, 25. One important point of the proof is that although the associated local well-posedness problem applies to objects on the timeslices, much of the technical work revolves around spacetime objects, in particular the spacetime curvature. The relevant spacetime and timeslice objects can then be related using standard elliptic estimates.

Using mostly the same set of ideas as in [17, D. Parlongue, in [19, proved an analogous breakdown criterion in the setting of a maximal time foliation with asymptotically flat timeslices. In addition, the breakdown condition was weakened from an $L^{\infty}$-bound to an iterated $L_{t}^{2} L_{x}^{\infty}$-bound:

$$
\|k\|_{L_{t}^{2} L_{x}^{\infty}(M)}+\|\nabla(\log n)\|_{L_{t}^{2} L_{x}^{\infty}(M)}<\infty .
$$

Furthermore, there have been additional results by Q. Wang toward an improved $L_{t}^{1} L_{x}^{\infty}$-criterion in the original CMC setting; see [26, 27]. 
1.3. Nonvacuum Spacetimes. The main question posed here is the following:

Do breakdown results analogous to that of Theorem 1 hold for Einsteinscalar and Einstein-Maxwell spacetimes?

In this paper, we will answer this question affirmatively.

The breakdown criteria in these nonvacuum cases remain largely the same as in the vacuum case. We retain the uniform bounds on the time foliation quantities $k$ and $\nabla(\log n)$. However, we must also impose a uniform bound on the now nontrivial matter field: the scalar field in the E-S setting, or the spacetime Maxwell 2-form in the E-M setting. This will be the only additional condition.

In the vacuum case of [17, the breakdown criterion in conjunction with the CMC gauge imply a wide range of priori controls with respect to the time foliation, all of which are essential for proving Theorem 1. These include, for example, uniform $L^{2}$-bounds for curvature along the timeslices, as well as uniform bounds for Sobolev constants on the timeslices. In the E-S and E-M cases, we will also need uniform bounds on the matter fields in order to achieve the a priori controls mentioned above. This will justify the modified breakdown criterion which we shall adopt.

We note that many of the elements which made the vacuum case so difficult will also make our nonvacuum cases similarly demanding. For instance, both [17] and the main result here are essentially "large data" results. Therefore, the only a priori "energy estimates" we will have are lower-order $L^{2}$-bounds on the curvature and the matter field. This greatly complicates the process behind controlling the local null geometry, since both the null injectivity radius and the Ricci coefficients will need to be controlled by these $L^{2}$-quantities. In particular, this will necessitate the use of the geometric Littlewood-Paley theory and the associated Besov estimates developed in [13] and further discussed in [21, Sec. 2.2].

The addition of matter fields also introduces a number of new difficulties:

- The spacetime Ricci curvature is now nontrivial.

- We must also deal with the coupling between the curvature and the matter field. An unavoidable consequence of this is that both the curvature and the matter field must be estimated concurrently.

- In the E-M case, there exist first-order terms in the wave equations satisfied by the curvature and Maxwell field which were not present in 17. These terms cannot be fully treated using only the techniques of [17] and its subsidiary papers. In particular, we will require a generalized representation formula for tensor wave equations; see [21, Ch. 5] and [22.

Another unfortunate reality is that the assumption of an E-V spacetime was pervasive throughout the entire proof of Theorem 1.3 As a consequence, most of the elements of the proof in the E-V case must in principle be redone. In particular, this includes controlling the local null geometry. In both the vacuum case of [17] and the nonvacuum cases presented here, the work on the null geometry comprises the most lengthy and technically involved portion of the overall proof. We will omit this portion of the proof from this paper, since it would more than double the length of this text. For details in this area, see [21, as well as its predecessors in the vacuum setting: [12, 14, 16, 19, 24, 25.

The following statement summarizes the main theorem of this paper:

\footnotetext{
${ }^{3}$ Notable exceptions include the geometric Littlewood-Paley theory of 13 and the KirchhoffSobolev parametrix of 15] (and its generalization in [21, Ch. 5] and [22]).
} 
Theorem 2. Suppose $(M, g, \Phi)$ is an $E$-S or $E$ - $M$ spacetime, given by

$$
M=\bigcup_{t_{0}<\tau<t_{1}} \Sigma_{\tau}, \quad t_{0}<t_{1}<0
$$

where each $\Sigma_{\tau}$ is a compact spacelike hypersurface of $M$ satisfying the constant mean curvature condition $\operatorname{tr} k \equiv \tau$, and where $\Phi$ denotes the matter field:

- In the $E-S$ case, $\Phi$ denotes the scalar field $\phi$.

- In the E-M case, $\Phi$ denotes the Maxwell 2-form F.

- Let $k$ and $n$ be as before in the statement of Theorem 1 .

In addition, let the quantity $\mathfrak{F}$ denote the following:

- The spacetime covariant differential D $\phi$ of $\phi$, in the E-S case.

- The Maxwell 2-form F, in the E-M case.

Assume the following breakdown criterion holds:

$$
\|k\|_{L^{\infty}(M)}+\|\nabla(\log n)\|_{L^{\infty}(M)}+\|\mathfrak{F}\|_{L^{\infty}(M)}<\infty .
$$

Then, $(M, g, \Phi)$ can be extended as an $E-S$ or $E-M$ (resp.) spacetime in the CMC gauge to some time $t_{1}+\epsilon$ for some $\epsilon>0$.

A more technically precise version is stated as Theorem [6 after a sufficient amount of background and notations have been developed.

1.4. Extensions and Open Problems. Finally, we discuss some possible extensions of Theorem 2, as well as some related open problems. The most immediate question is whether Theorem 2 can be similarly adapted to other related classes of nonvacuum spacetimes, such as Einstein-Klein-Gordon and Einstein-Yang-Mills spacetimes. The answer is affirmative for the above two spacetimes in this same CMC gauge framework. The class of techniques presented in this text can be applied directly to the Einstein-Klein-Gordon case. This is also mostly true for the Einstein-Yang-Mills setting; however, in this case, we will also require vector bundle generalizations of many of the tools used here to handle the Yang-Mills curvature both in a covariant and in a gauge-invariant fashion. 4

Whether analogous breakdown criteria can be stated and proved for less similar classes of nonvacuum spacetimes, such as the Einstein-Euler and the EinsteinVlasov models, remains an open question. For such matter fields, one would likely require other tools besides the representation formula for tensor wave equations in order to derive higher-order energy inequalities.

Another variation of Theorem 2 which should be possible is the case of a maximal foliation with asymptotically flat timeslices for various nonvacuum spacetimes. The adaptations to the theorem statement and proof should then be analogous to [19. Note that one must also amend the energy norms for the curvature and the matter fields in this setting along the lines of [19].

One more potential direction of investigation involves the possible weakening of the $L^{\infty}$-bounds in the breakdown criterion to slightly weaker iterated bounds, such as the $L_{t}^{2} L_{x}^{\infty}$ bounds found in [19]. A further improvement to $L_{t}^{1} L_{x}^{\infty}$-bounds along the lines of [26, 27] is also expected to be achievable.

A more difficult open question related to the topic at hand is the well-known CMC conjecture. In this case, the desired conclusion is that the spacetimes of

\footnotetext{
${ }^{4}$ In particular, this includes the vector bundle extension of the generalized Kirchhoff-Sobolev parametrix; see [21, Sec. 5.3] and [22, Sec. 5].
} 
Theorems 1 and 2 can in fact be continued up to time 0. Finally, another related conjecture is the bounded $L^{2}$-curvature conjecture, which in principle states that a local well-posed theory exists based on control of the $L^{2}$-norms of the curvature. There has been significant recent progress in resolving this question.

Acknowledgements. The author wishes to thank Professor Sergiu Klainerman for suggesting this problem and for hours of discussions throughout the preparation of this work. Thanks also extends to Professor Igor Rodnianski for his insights. In addition, the author thanks Qian Wang for helpful technical discussions.

\section{Tensorial Notations}

In this section, we construct some notations for the various types of tensor fields we will encounter. The main objective of these notations is to highlight the covariant structures present in our setting. 5 Although such structures were also pertinent to the analogous vacuum problem of [17, as well as other related works (for example, [7, 12, 19]), they were described more implicitly in those texts. We develop the new and more explicit notations here in the hopes of promoting a more conceptual outlook on the objects of our analysis. The conventions used here will be an abridged version of that of 21.

2.1. Tensor Fields. Let $M$ denote an arbitrary smooth manifold. First, we set the notations for standard tensor bundles over $M$ :

- Let $T_{s}^{r} M$ denote the rank- $(r, s)$ tensor bundle of $M$.

- Let $S^{s} M$ denote the bundle of all fully symmetric elements of $T_{s}^{0} M$.

- Let $\Lambda^{s} M$ denote the bundle of all fully antisymmetric elements of $T_{s}^{0} M$, i.e., the exterior bundle of degree $s$ on $M$.

In general, for a smooth vector bundle $\mathcal{V}$ over $M$, we define the following:

- Given $p \in M$, let $\mathcal{V}_{p}$ denote the fiber of $\mathcal{V}$ at $p$. The lone exception is for tangent spaces, for which we use the more standard notation

$$
T_{p} M=\left(T_{0}^{1} M\right)_{p} .
$$

- Let $\Gamma \mathcal{V}$ denote the smooth sections of $\mathcal{V}$. For example, $\Gamma T_{s}^{r} M$ and $\Gamma \Lambda^{s}$ are the spaces of rank- $(r, s)$ tensor fields and $s$-forms on $M$, respectively.

For convenience, we also define the abbreviations

$$
\mathfrak{X}(M)=\Gamma T_{0}^{1} M, \quad \mathfrak{X}^{*}(M)=\Gamma T_{1}^{0} M, \quad \Gamma \mathcal{T} M=\bigcup_{r, s \geq 0} \Gamma T_{s}^{r} M,
$$

i.e., the space of vector fields on $M$, the space of 1 -forms on $M$, and the space of all tensor fields on $M$, respectively.

When convenient, we will adopt standard index notation to represent tensor fields with respect to local frames and dual coframes. In many instances, these frames satisfy additional conditions, e.g., coordinate, orthonormal, or null frames. In accordance with Einstein summation notation, indices repeated in both superscript and subscript represent a summation of components, i.e., a contraction. In addition, we will use capital letters to denote collections of indices. Moreover, repeated capital letters signify summations over all represented indices.

\footnotetext{
${ }^{5}$ By covariant structures, we mean vector bundles with metrics and compatible connections.
} 
Furthermore, when writing in index notation, we let $\mathfrak{A}_{\alpha \beta}[\cdot]$ denote an unnormalized antisymmetrization of the indices $\alpha$ and $\beta$ of the indexed quantity within the bracket. For example, if $S \in \Gamma T_{1}^{1} M$ and $T \in \Gamma T_{2}^{0} M$, then

$$
\mathfrak{A}_{\beta \gamma}\left[S^{\alpha}{ }_{\beta} T_{\gamma \delta}\right]=S^{\alpha}{ }_{\beta} T_{\gamma \delta}-S_{\gamma}^{\alpha} T_{\beta \delta}=2 S^{\alpha}{ }_{[\beta} T_{\gamma] \delta} .
$$

Although the standard bracket notation on the right-hand side of (7) achieves the same effect, the $\mathfrak{A}$-notation will be useful for larger expressions with multiple antisymmetries, in which the bracket notation would be confusing or ambiguous.

In the case that $M$ is Riemannian or Lorentzian, with metric $g \in \Gamma S^{2} M$, then contravariant and covariant components are equivalent, and we will often write $T^{r+s} M$ for $T_{s}^{r} M$. 6 Moreover, given a field $T \in \Gamma T^{r} M$, we define $R[T] \in \Gamma T^{r+2} M$ to be the covariant derivative commutator

$$
R_{\alpha \beta}[T]_{I}=D_{\alpha \beta} T_{I}-D_{\beta \alpha} T_{I} .
$$

We will also follow these standard traditions within index notation:

- We denote both the Ricci and scalar curvatures of $(M, g)$ by $R$, but with the appropriate number of indices.

- If $M$ is oriented, then the volume form is denoted $\epsilon$.

Finally, if $(M, g)$ is Riemannian, we can define natural tensor norms by

$$
|\cdot|: \Gamma \mathcal{T} M \rightarrow C^{\infty}(M), \quad|\Psi|=\left(\Psi^{I} \Psi_{I}\right)^{\frac{1}{2}} .
$$

We can then define the obvious $L^{p}$-norms, $1 \leq p \leq \infty$, for such tensor fields.

2.2. Foliations. Next, we briefly discuss a rather general situation of a 1-parameter foliation of Riemannian manifolds. Let $(M, g)$ denote a Lorentzian manifold, and let $N$ denote a smooth submanifold of $M$. Let $f \in C^{\infty}(N)$, mapping onto a possibly infinite open interval $\mathcal{I}$, with $d f$ nonvanishing. For each $x \in \mathcal{I}$, we assume

$$
\Sigma_{x}=\{p \in M \mid f(p)=x\}
$$

is a Riemannian submanifold of $M$. In other words, we can write

$$
N=\bigcup_{x \in \mathcal{I}} \Sigma_{x}
$$

as a 1-parameter foliation of Riemannian submanifolds.

In future sections, this situation will arise in the following instances:

- Time foliations of spacetime: In this case, $N=M$, and $f$ is a time function on $M$ which assigns to each a point a time value; see Sec. 3.1

- Spherical foliations of regular null cones: In this case, $N$ is a smooth portion of a past null cone in $M$, and $f$ foliates $N$ into spherical cross-sections; see Sec. 4.2. In particular, $N$ is null and hence is not pseudo-Riemannian.

Since it will be convenient to have some common notations for both cases, we commit to this abstract development here.

We begin by defining the following bundles over $N$ :

- A tensor $w \in\left(T^{r} M\right)_{q}$ at $q \in \Sigma_{x}$ is horizontal iff $w$ is tangent to $\Sigma_{x}$.

- We denote by $\underline{T}^{k} N$ the horizontal bundle over $N$ of all horizontal tensors of total rank $k$ at every $q \in N$.

- We denote by $\bar{T}^{l} N$ the extrinsic bundle over $N$ of all tensors in $M$ of total rank $l$ at every $q \in N$, i.e., the restriction of $T^{l} M$ to $N$.

\footnotetext{
${ }^{6}$ For example, the Riemann curvature $R$ of $(M, g)$ is an element of $\Gamma T^{4} M$.
} 
- The mixed bundle $\bar{T}^{l} \underline{T}^{k} N$ over $N$ is defined to be the tensor product bundle

$$
\underline{T}^{k} \bar{T}^{l} N=\underline{T}^{k} N \otimes \bar{T}^{l} N .
$$

For convenience, we adopt the notations

$$
\Gamma \underline{\mathcal{T}} N=\bigcup_{k \geq 0} \Gamma \underline{T}^{k} N, \quad \Gamma \overline{\mathcal{T}} N=\bigcup_{l \geq 0} \Gamma \bar{T}^{l} N, \quad \Gamma \underline{\mathcal{T}} \overline{\mathcal{T}} N=\bigcup_{k, l \geq 0} \Gamma \underline{T}^{k} \bar{T}^{l} N,
$$

i.e., the spaces of horizontal, extrinsic, and mixed tensor fields on $N$. We also define

$$
\underline{\mathfrak{X}}(N)=\Gamma \underline{T}^{1} N, \quad \overline{\mathfrak{X}}(N)=\Gamma \bar{T}^{1} N .
$$

Moreover, we adopt the following general indexing conventions:

- Horizontal indices will be denoted using Latin letters.

- Extrinsic indices will be denoted using Greek letters.

- Collections of extrinsic indices will be denoted using capital Latin letters.

For example, the pullback to $N$ of $g$ is an element of $\Gamma \bar{T}^{2} N$, while the induced metrics $\gamma$ on the $\Sigma_{x}$ 's is in $\Gamma \underline{T}^{2} N$. Note that $\gamma$ induces positive-definite bundle metrics $\langle\cdot, \cdot\rangle$ on the horizontal bundles $\underline{T}^{k} N$ via full metric contraction. Similarly, the restriction of $g$ to $N$ induces bundle metrics $\langle\cdot, \cdot\rangle$ on the extrinsic bundles $\bar{T}^{l} N$. These in turn naturally induce "product" bundle metrics on $\underline{T}^{k} \bar{T}^{l} N$.

Next, let $D$ denote the Levi-Civita connection on $(M, g)$. Recall from standard theory that $D$ induces a connection $\bar{D}$ on the extrinsic bundles $\bar{T}^{l} N$. Moreover, $\bar{D}_{X} g \equiv 0$ by definition, hence $\bar{D}$ is compatible with the extrinsic bundle metrics. 0

We can also naturally define a connection $\nabla$ on the horizontal bundles via projections. In particular, given $X \in \mathfrak{X}(N)$, then $\nabla$ satisfies the following properties:

- If $f \in C^{\infty}(N)$, then $\nabla_{X} f=X f$, as usual.

- If $Y \in \underline{\mathfrak{x}}(N)$, then $\nabla_{X} Y$ is the projection onto the $\Sigma_{x}$ 's of $\bar{D}_{X} Y$.

- If $A \in \bar{\Gamma} \underline{T}^{k} N$ is fully covariant, and $Y_{1}, \ldots, Y_{k} \in \underline{\mathfrak{x}}(N)$, then

$$
\begin{gathered}
\nabla_{X} A\left(Y_{1}, \ldots, Y_{k}\right)=X\left[A\left(Y_{1}, \ldots, Y_{k}\right)\right]-A\left(\nabla_{X} Y_{1}, Y_{2}, \ldots, Y_{k}\right) \\
-\ldots-A\left(Y_{1}, \ldots, Y_{k-1}, \nabla_{X} Y_{k}\right) .
\end{gathered}
$$

This definition of $\nabla$ generalizes the usual Levi-Civita connections on the $\Sigma_{x}$ 's to also include non-horizontal derivatives tangent to $N$. Since $\nabla_{X} \gamma \equiv 0$ for any $X \in \mathfrak{X}(N)$, then $\nabla$ remains compatible with the horizontal bundle metrics.

We can also define mixed connections $\bar{\nabla}$ on the mixed bundles using the above connections $\nabla$ and $\bar{D}$. On $\Gamma \underline{T}^{k} \bar{T}^{l} N$, we define $\bar{\nabla}$ to be the unique connection satisfying the following Leibniz identity for decomposable fields:

$$
\bar{\nabla}_{X}(A \otimes B)=\nabla_{X} A \otimes B+A \otimes \bar{D}_{X} B, \quad X \in \mathfrak{X}(N), A \in \Gamma \underline{T}^{k} N, B \in \Gamma \bar{T}^{l} N .
$$

In other words, $\bar{\nabla}$ behaves like $\nabla$ and $\bar{D}$ on horizontal and extrinsic components, respectively. Clearly, $\bar{\nabla}$ is compatible with the above mixed bundle metrics.

Remark. More explicitly, if $A \in \Gamma \underline{T}^{k} \bar{T}^{l} N ; X \in \mathfrak{X}(N) ; Y_{1}, \ldots, Y_{k} \in \underline{\mathfrak{X}}(N)$; and $Z_{1}, \ldots, Z_{l} \in \overline{\mathfrak{X}}(N)$; then $\bar{\nabla}_{X} A\left(Y_{1}, \ldots, Y_{k} ; Z_{1}, \ldots, Z_{l}\right)$ is assigned the value

$$
\begin{aligned}
& X\left[A\left(Y_{1}, \ldots, Y_{k} ; Z_{1}, \ldots, Z_{l}\right)\right]-A\left(\nabla_{X} Y_{1}, Y_{2}, \ldots, Y_{k} ; Z_{1}, \ldots Z_{l}\right)-\ldots \\
& \quad-A\left(Y_{1}, \ldots, Y_{k-1}, \nabla_{X} Y_{k} ; Z_{1}, \ldots, Z_{l}\right)-A\left(Y_{1}, \ldots, Y_{k} ; \bar{D}_{X} Z_{1}, Z_{2}, \ldots Z_{l}\right) \\
& \quad-\ldots-A\left(Y_{1}, \ldots, Y_{k} ; Z_{1}, \ldots, Z_{l-1}, \bar{D}_{X} Z_{l}\right) .
\end{aligned}
$$

\footnotetext{
${ }^{7}$ Technically, by $\bar{D}_{X} g$, we mean $\bar{D}_{X}$ acting on the restriction of $g$ to $N$.
} 
We also set the following notations:

- For $A \in \Gamma \underline{T}^{k} N$, define $\nabla A \in \Gamma \underline{T}^{k+1} N$ to map $X \in \underline{\mathfrak{X}}(N)$ to $\nabla_{X} A$.

- For $A \in \Gamma \underline{T}^{k} \bar{T}^{l} N$, define $\bar{\nabla} A \in \Gamma \underline{T}^{k+1} \bar{T}^{l} N$ to map $X \in \underline{\mathfrak{x}}(N)$ to $\bar{\nabla}_{X} A$.

- Horizontal and mixed Laplacians are defined in the usual fashion:

$$
\Delta=\gamma^{a b} \nabla_{a b}, \quad \bar{\Delta}=\gamma^{a b} \bar{\nabla}_{a b} .
$$

For further details involving the above constructions, see [21, Sec. 1.2].

Remark. In contrast, the wave operator $g^{\alpha \beta} D_{\alpha \beta}$ on $(M, g)$ is denoted $\square$.

The fundamental properties are the Leibniz rules satisfied by the connections and the compatibility between the connections and metrics. These justify integration by parts operations involving $\bar{\nabla}$-derivatives, seen in $15,17,21,22$ and later in this paper. This was used implicitly in [15, 17] and was discussed in detail in [21, 22.

2.3. Normal Transport. Assume the same foliation setting as before. For each point $p \in \Sigma_{x} \subseteq N$, there is a unique direction in $N$ which is normal to $\Sigma_{x}$. Thus, we can define the "normalized normal" vector field $Z \in \mathfrak{X}(N)$ such that $Z$ points in the direction in $N$ normal to the $\Sigma_{x}$ 's and satisfies $Z f \equiv 1$.

We can naturally define quantities via transport along the integral curves of $Z$. For instance, given a locally defined function on some $\Sigma_{y}$, we can define a corresponding local function on another $\Sigma_{x}$ via the diffeomorphisms induced by the above transport. In particular, given a coordinate system $\left(y^{1}, y^{2}\right)$ in $\Sigma_{y}$, we can define a transported coordinate system on the other $\Sigma_{x}$ 's.

Similarly, we can transport horizontal tensors along the integral curves of $Z$. This induces the standard definition of "Lie derivatives" of horizontal tensor fields. More explicitly, for any $A \in \Gamma \underline{\mathcal{T}} N$, its "normal Lie derivative" $\mathcal{L}_{f} A$ is defined

$$
\left.\mathcal{L}_{f} A\right|_{p}=\lim _{\delta \rightarrow 0} \frac{f_{x+\delta, x}^{*}\left(\left.A\right|_{f_{x, x+\delta}(p)}\right)-\left.A\right|_{p}}{\delta}, \quad p \in \Sigma_{x} \subseteq N,
$$

where $f_{x+\delta, x}$ is the flow from $\Sigma_{x+\delta}$ to $\Sigma_{x}$ via the integral curves of $Z$, and where $f_{x+\delta, x}^{*}$ denotes the "push-forward" of tensors through $f_{x+\delta, x}$.

Remark. The development described here differs from that of [17, 21, which expressed similar notions entirely in terms of transported coordinate systems. Notice that the normal Lie derivatives of (16), which are tensorial and invariant, coincide with the $f$-coordinate derivatives for such transported coordinate systems.

Finally, we define the analogous "normalized normal" covariant derivatives: for fields $A \in \Gamma \mathcal{I} N$ and $B \in \Gamma \mathcal{\mathcal { T }} \overline{\mathcal{T}} N$, we define

$$
\nabla_{f} A=\nabla_{Z} A, \quad \bar{\nabla}_{f} B=\bar{\nabla}_{Z} B .
$$

2.4. The Einstein Equations. Assume now that $(M, g)$ is a $(1+3)$-dimensional connected, oriented, and time-oriented Lorentzian manifold, with Levi-Civita connection $D$ and Riemann curvature $R$. In addition, let $\mathfrak{f}$ denote a collection of prescribed "matter fields" on $M$, satisfying the equations of their respective theories. The Einstein equations on $M$ are given in index notation by

$$
G_{\alpha \beta}=R_{\alpha \beta}-\frac{1}{2} \cdot R \cdot g_{\alpha \beta}=Q_{\alpha \beta},
$$


where $Q \in \Gamma S^{2} M$ denotes the energy-momentum tensor of the matter fields. Recall that the Ricci curvature can be expressed in terms of the matter fields:

$$
R_{\alpha \beta}=Q_{\alpha \beta}-\frac{1}{2} g_{\alpha \beta} g^{\mu \nu} Q_{\mu \nu} .
$$

Moreover, recall that $Q$ is both symmetric and divergence-free.

In this text, we will consider the following settings for matter fields:

- Vacuum: There are no matter fields, so both $Q$ and Ric vanish.

- Scalar field: In this model case, $\mathfrak{f}$ is given as a scalar field $\phi \in C^{\infty}(M)$, which satisfies the linear homogeneous covariant wave equation

The energy-momentum tensor and Ricci curvature are given by

$$
\square_{g} \phi=0 .
$$

$$
Q_{\alpha \beta}=D_{\alpha} \phi D_{\beta} \phi-\frac{1}{2} g_{\alpha \beta} D^{\mu} \phi D_{\mu} \phi, \quad R_{\alpha \beta}=D_{\alpha} \phi D_{\beta} \phi .
$$

- Maxwell field: In the model electromagnetic case, $\mathfrak{f}$ is given as a Maxwell field $F \in \Gamma \Lambda^{2} M$, which satisfies the linear homogeneous Maxwell equations

$$
D^{\beta} F_{\alpha \beta} \equiv 0, \quad D_{\alpha} F_{\beta \gamma}+D_{\beta} F_{\gamma \alpha}+D_{\gamma} F_{\alpha \beta} \equiv 0 .
$$

The energy-momentum tensor and Ricci curvature are given by

$$
Q_{\alpha \beta}=R_{\alpha \beta}=F_{\alpha \mu} F^{\beta \mu}-\frac{1}{4} g_{\alpha \beta} F^{\mu \nu} F_{\mu \nu} .
$$

We remark that in the above settings, $Q$ satisfies both the positive energy condition and the strong energy condition, i.e., $Q(X, Y) \geq 0$ and $\operatorname{Ric}(X, Y) \geq 0$ for all future causal $X, Y \in \mathfrak{X}(M)$. Moreover, by direct calculations, we can compute

$$
\begin{aligned}
D^{\alpha} R_{\alpha \beta \gamma \delta} & \equiv 0, \\
D^{\alpha} R_{\alpha \beta \gamma \delta} & =-\mathfrak{A}_{\gamma \delta}\left[D_{\gamma} \phi D_{\delta \beta} \phi\right], \\
D^{\alpha} R_{\alpha \beta \gamma \delta} & =-F_{\beta}{ }^{\mu} D_{\mu} F_{\gamma \delta}-\mathfrak{A}_{\gamma \delta}\left[F_{\gamma}{ }^{\mu} D_{\delta} F_{\beta \mu}+\frac{1}{2} g_{\beta \gamma} F^{\mu \lambda} D_{\delta} F_{\mu \lambda}\right]
\end{aligned}
$$

in the E-V, E-S, E-M settings, respectively. 8

\section{The CMC Breakdown and Cauchy Problems}

For the breakdown problem at hand, we are given an existing solution of the Einstein equations, i.e., an E-S or E-M spacetime "existing on a finite time interval". Our goal will be to "extend the solution further in time" given that certain criteria hold on this existing solution. In this section, we will make precise the above informal expressions. This essentially requires smoothly assigning to each point of the spacetime a "time value" and then foliating the spacetime into "timeslices", that is, hypersurfaces of constant time value.

This objective will be achieved by defining global time functions and the various fundamental objects associated with its resulting foliation. In addition, for the breakdown problem, we will adopt the CMC gauge condition as well.

Throughout the remainder of this paper, we will always let $(M, g)$ denote a $(1+3)$-dimensional connected, oriented, and time-oriented Lorentzian manifold, with Levi-Civita connection $D$ and curvature $R$.

\footnotetext{
${ }^{8}$ See (7).
} 
3.1. Time Foliations. We define a time function on $M$ to be a map $t \in C^{\infty}(M)$ satisfying the following conditions:

- The spacetime gradient of $t$ is everywhere past timelike.

- Every nonempty level set of $t$ is a Cauchy hypersurface of $M$, i.e., every inextendible causal curve in $M$ intersects each level set of $t$ exactly once.

From the first property, we see $t$ is strictly increasing along all future directions. Moreover, the second property implies that $(M, g)$ is globally hyperbolic.

For $\tau \in \mathbb{R}$ and an interval $I \subseteq \mathbb{R}$, define

$$
\Sigma_{\tau}=\{z \in M \mid t(z)=\tau\}, \quad \Sigma_{I}=\{z \in M \mid t(z) \in I\} .
$$

The family $\left\{\Sigma_{\tau}\right\}$ of spacelike Cauchy hypersurfaces defines a time foliation of $M$,

$$
M=\bigcup_{\tau \in \mathcal{I}} \Sigma_{\tau}
$$

where $\mathcal{I}$ is an interval in $\mathbb{R}$, and where $t$ maps onto $\mathcal{I}$. We will also impose the following assumptions: the interval $\mathcal{I}$ is finite, and the $\Sigma_{\tau}$ 's are compact.

Remark. Any two slices $\Sigma_{\tau_{1}}, \Sigma_{\tau_{2}}$, where $\tau_{1}, \tau_{2} \in \mathcal{I}$, are in fact diffeomorphic, since any $p_{1} \in \Sigma_{\tau_{1}}$ can be canonically identified with the point $p_{2} \in \Sigma_{t_{2}}$ on the integral curve of grad $t$ through $p_{1}$.

We adopt the notations developed in the Section 2.2 to describe the foliation (27), with $N=M$ and $f=t$. In addition, we adopt the following indexing conventions:

- Greek letters refer to all components in $M$, ranging from 0 to 3.

- Latin letters refer to horizontal components, ranging from 1 to 3.

- For implicit index summations, repeating Greek indices are summed from 0 to 3, while repeating Latin indices are summed from 1 to 3 .

Also, let $\gamma, \nabla$, and $\mathcal{R}$ denote the horizontal metrics, connections, and curvatures. Next, we define the following basic objects of interest:

- Let $n \in C^{\infty}(M)$ denote the lapse function, given by

$$
n=|g(\operatorname{grad} t, \operatorname{grad} t)|^{-\frac{1}{2}}>0 .
$$

- Let $T \in \mathfrak{X}(M)$ denote the future unit normal to the $\Sigma_{\tau}$ 's, i.e.,

$$
T=-n \cdot \operatorname{grad} t .
$$

- Let $k \in \Gamma \underline{T}^{2} M$ be the future second fundamental form of the $\Sigma_{\tau}$ 's:

$$
k(X, Y)=-g\left(D_{X} T, Y\right), \quad X, Y \in \underline{\mathfrak{X}}(M) .
$$

Recall that $k$ is symmetric and can be decomposed into trace and traceless parts:

$$
\operatorname{tr} k=\gamma^{i j} k_{i j} \in C^{\infty}(M), \quad \hat{k}=k-\frac{1}{3}(\operatorname{tr} k) \gamma \in \Gamma \underline{T}^{2} M .
$$

Recall also that $\operatorname{tr} k$ corresponds to the mean curvature of the $\Sigma_{\tau}$ 's in $M$.

We can relate spatial derivatives of $\mathcal{R}$ and $k$ to spacetime derivatives of $R$. First, the Gauss equation, expressed in index notation in terms of $\mathcal{R}$ and $k$, becomes

$$
R_{i j l m}=\mathcal{R}_{i j l m}-k_{j l} k_{i m}+k_{i l} k_{j m} .
$$

Similarly, the Codazzi equations and a direct calculation imply

$$
\nabla^{j} k_{i j}=R_{\alpha i} T^{\alpha}+\nabla_{i}(\operatorname{tr} k), \quad \nabla_{[i} k_{j] l}=R_{\alpha l i j} T^{\alpha} .
$$


In this setting, the "normalized normal" vector field $Z$ is given by $Z=n T$. 9 In addition, the quantities $\gamma$ and $k$ satisfy the evolution equations

$$
\begin{aligned}
\mathcal{L}_{t} \gamma & =-2 n k, \\
\mathcal{L}_{t} k_{i j} & =-\nabla_{i j} n+n R_{i \alpha j \beta} T^{\alpha} T^{\beta}-n k_{i l} k_{j}{ }^{l},
\end{aligned}
$$

where $\mathcal{L}_{t}$ denotes the normal Lie derivative of (16).

We can naturally define an associated Riemannian metric $h$ on $M$ by

$$
h(X, Y)=g(X, Y)+2 g(T, X) g(T, Y), \quad X, Y \in \mathfrak{X}(M) .
$$

In other words, we define $h$ to act like $g$ on the horizontal components, and we simply invert the sign of the normal timelike component. It is easy to see that $h$ is Riemannian, and that $h$ agrees with $\gamma$ for horizontal vector fields. From now on, tensor norms on $M$ will be defined with respect to $h$.

The following proposition states that the Cauchy-Schwarz inequality continues to hold with respect to contractions by $g$ :

Proposition 3. Let $\Phi, \Psi \in \Gamma \mathcal{T} M$, and let $\Phi \cdot \Psi \in \Gamma \mathcal{T} M$ denote a tensor field obtained by taking zero or more contractions and $g$-contractions of $\Phi \otimes \Psi$. Then, $|\Phi \cdot \Psi| \lesssim|\Phi||\Psi|$, where the constant depends on the number of contractions. 10

Proof. See [21, Prop. 4.1].

Let $\pi={ }^{(T)} \pi \in \Gamma S^{2} M$ denote the deformation tensor of $T$ :

$$
\pi(X, Y)=\mathcal{L}_{T} g(X, Y)=g\left(D_{X} T, Y\right)+g\left(D_{Y} T, X\right), \quad X, Y \in \mathfrak{X}(M) .
$$

By direct computation, we can relate $\pi$ to $n$ and $k$ : for any $X, Y \in \underline{\mathfrak{X}}(M)$,

$$
\pi(T, T) \equiv 0, \quad \pi(X, Y)=-2 k(X, Y), \quad \pi(T, X)=X(\log n) .
$$

In other words, $\pi$ can be thought of as a spacetime object which contains precisely the same information as the horizontal objects $k$ and $\nabla(\log n)$.

One reason for the importance of the deformation tensor is that it bounds a number of essential quantities related to the time foliation, the most important of which are demonstrated by (38). We also have from [21, Prop. 4.2] the bounds

$$
|D T| \lesssim|\pi|, \quad|D h| \lesssim|\pi|
$$

For instance, the following calculus estimate is a direct consequence of (39):

Proposition 4. The following calculus estimate holds:

$$
\bar{\nabla}|\Psi|^{p} \lesssim|\Psi|^{p-1}|\bar{\nabla} \Psi|+|\pi||\Psi|^{p}, \quad p \geq 1, \Psi \in \Gamma \mathcal{\mathcal { T }} \overline{\mathcal{T}} \Sigma_{\tau} .
$$

Proof. Recalling the Leibniz and metric compatibility properties of the mixed covariant differential $\bar{\nabla}$, along with Proposition 3, we compute

$$
\begin{aligned}
\bar{\nabla}|\Psi|^{p} & =\frac{p}{2}[h(\Psi, \Psi)]^{\frac{p-2}{2}} \bar{\nabla}[h(\Psi, \Psi)] \\
& \leq p|\Psi|^{p-2} \cdot|\Psi||\bar{\nabla} \Psi|+\frac{p}{2}|\Psi|^{p-2}|D h||\Psi|^{2} .
\end{aligned}
$$

The proof is completed by applying (39).

Lastly, we note the following coarea formula:

\footnotetext{
${ }^{9}$ See Section 2.3

${ }^{10} \mathrm{By} A \lesssim B$, we mean $A \leq C B$ for some positive constant $C$.
} 
Proposition 5. If $\Omega$ is an open subset of $M$ and $\mathcal{J}$ is a subinterval of $\mathcal{I}$, then

$$
\int_{\Sigma_{\mathcal{J}} \cap \Omega} \phi=\int_{\mathcal{J}}\left(\int_{\Sigma_{\tau} \cap \Omega} n \cdot \phi\right) d \tau
$$

for any integrable $\phi \in C^{\infty}(\Omega)$.

Proof. See [21, Prop. 4.4].

3.2. The Main Theorem. In terms of describing the Einstein equations as a system of partial differential equations, the above prescription of a time function and foliation leaves us with still another degree of freedom: a gauge condition satisfied by the foliation. In this text, we will adopt the constant mean curvature, or $C M C$, gauge, as was done in [17.

To be much more precise, we say that the spacetime $(M, g)$, along with a time function $t \in C^{\infty}(M)$ and negative real numbers $t_{0}<t_{1}<0$, satisfy the condition $(\mathbf{C M C})_{t, t_{0}, t_{1}}$ iff the following conditions hold:

- The total timespan $\mathcal{I}=t(M)$ is precisely the interval $\left(t_{0}, t_{1}\right)$.

- The timeslices $\Sigma_{\tau}$ are compact.

- The CMC condition holds, that is, $\operatorname{tr} k=t$ everywhere on $M$.

We are now ready to state the precise main theorem of this text:

Theorem 6. Suppose $(M, g, \Phi)$ is an Einstein-scalar or an Einstein-Maxwell spacetime, where $\Phi$ denotes the matter field $\phi \in C^{\infty}(M)$ or $F \in \Gamma \Lambda^{2} M$, corresponding to the E-S and E-M cases, respectively. In addition, suppose the following hold:

- The condition (CMC) $)_{t, t_{0}, t_{1}}$ holds for $(M, g)$.

- The following "breakdown criterion" holds for some constant $C_{0}>0$,

$$
\|k\|_{L^{\infty}(M)}+\|\nabla(\log n)\|_{L^{\infty}(M)}+\|\mathfrak{F}\|_{L^{\infty}(M)} \leq C_{0},
$$

where $\mathfrak{F}$ denotes either $D \phi$ or $F$ in the $E-S$ and $E-M$ cases, respectively.

Then, $(M, g, \Phi)$ can be extended past time $t_{1}$ as a CMC foliation. In other words, there is an Einstein-scalar or Einstein-Maxwell (resp.) spacetime $\left(M_{\star}, g_{\star}, \Phi_{\star}\right)$ satisfying (CMC $)_{t_{\star}, t_{0}, t_{1}+\epsilon}$, where $t_{\star}$ is a time function on $M_{\star}, \epsilon>0$, and $t_{1}+\epsilon<0$, such that the following statements hold:

- There exists an isometric imbedding i from $(M, g)$ into $\left(M_{\star}, g_{\star}\right)$.

- The maps $t$ and $t_{\star}$ correspond with respect to $i$, i.e., for every $t_{0}<\tau<t_{1}$,

$\mathfrak{i}\left(\Sigma_{\tau}\right)=\left\{q \in M_{\star} \mid t_{\star}(q)=\tau\right\}, \quad \mathfrak{i}(M)=\left\{q \in M_{\star} \mid t_{0}<t_{\star}(q)<t_{1}\right\}$.

In particular, we have $t=t_{\star} \circ \mathfrak{i}$.

- The matter fields $\Phi$ and $\Phi_{\star}$ also correspond with respect to i, i.e., $\Phi=\mathfrak{i}^{*} \Phi_{\star}$.

The goal of the remainder of the paper will be to prove Theorem 6

3.3. The Cauchy Problem. We now give an explicit formulation of the Cauchy problems for the E-S and E-M equations in the CMC gauge. We will then state a basic local well-posedness result for this problem.

A 4-tuple $\left(\Sigma_{0}, \gamma_{0}, k_{0}, \mathfrak{f}_{0}\right)$ will be called an "admissible initial data set" for the Einstein-scalar or Einstein-Maxwell equations iff the following hold:

- $\left(\Sigma_{0}, \gamma_{0}\right)$ is a 3-dimensional compact oriented Riemannian manifold.

- The field $k_{0}$ is an element of $\Gamma S^{2} \Sigma_{0}$. 11

\footnotetext{
${ }^{11} k_{0}$ is to be interpreted as a "second fundamental form" for $\Sigma_{0}$.
} 
- The "mean curvature" tr $k_{0}$ has a constant value $\tau_{0}<0$ on all of $\Sigma_{0}$.

- The field $\mathfrak{f}_{0}$ corresponds to initial data for the given matter field. In the E-S case, this is expressed as a pair $\phi_{0}, \phi_{1} \in C^{\infty}\left(\Sigma_{0}\right)$, while in the E-M case, this is given as a pair $E_{0}, H_{0} \in \mathfrak{X}^{*}\left(\Sigma_{0}\right)$.

- Letting $\nabla$ and $\mathcal{R}_{0}$ denote the Levi-Civita connection and the curvature for $\Sigma_{0}$, respectively, then in the E-S case, the following constraints hold,

$$
-\phi_{1} \nabla_{i} \phi_{0}=\nabla^{j}\left(k_{0}\right)_{i j}, \quad\left|\nabla \phi_{0}\right|^{2}+\phi_{1}^{2}=\mathcal{R}_{0}-\left|k_{0}\right|^{2}+\tau_{0}^{2},
$$

while in the E-M case, the following constraints hold,

$$
-\epsilon_{i}{ }^{j l}\left(E_{0}\right)_{j}\left(H_{0}\right)_{l}=\nabla^{j}\left(k_{0}\right)_{i j}, \quad\left|E_{0}\right|^{2}+\left|H_{0}\right|^{2}=\mathcal{R}_{0}-\left|k_{0}\right|^{2}+\tau_{0}^{2} .
$$

- In the E-M case, the "matter field" $\mathfrak{f}_{0}$ solves the additional constraints

$$
\nabla^{i}\left(E_{0}\right)_{i} \equiv 0, \quad \nabla^{i}\left(H_{0}\right)_{i} \equiv 0 .
$$

Remark. We make the following remarks about the above definition:

- The symbols $|\cdot|$ in (41), (42) denote the $\gamma_{0}$-tensor norm. The volume form $\epsilon_{i j k}$ in (42) is that of $\left(\Sigma_{0}, \gamma_{0}\right)$, with respect to a chosen orientation of $\Sigma_{0}$.

- Note that (41) and (42) correspond to the E-S and E-M (resp.) constraint equations in the CMC gauge. Similarly, (43) corresponds to the standard constraints for the Maxwell equations.

- If $\phi$ and $F$ are the desired "spacetime fields" for which we wish to solve, then $\phi_{0}$ and $\phi_{1}$ correspond to the values $\phi$ and $\mathcal{L}_{t} \phi$ on $\Sigma_{0}$, while $E_{0}$ and $H_{0}$ correspond to the electromagnetic decomposition of $F$ on $\Sigma_{0}$.

Now, if we are given an admissible initial data set $\left(\Sigma_{0}, \gamma_{0}, k_{0}, \mathfrak{f}_{0}\right)$ in either the E-S or the E-M setting, then the goal will be to solve for a triple $(M, g, \Phi)$, along with maps $\mathfrak{i}: \Sigma_{0} \rightarrow M$ and $t \in C^{\infty}(M)$, where the following hold:

- $(M, g)$ is a $(1+3)$-dimensional globally hyperbolic Lorentzian manifold.

- There exists a time function $t$ on $M$, along with constants $t_{0}, t_{1} \in \mathbb{R}$, where $t_{0}<\tau_{0}<t_{1}<0$, such that $(M, g)$ satisfies the condition $(\mathbf{C M C})_{t, t_{0}, t_{1}}$.

- The map $\mathfrak{i}$ is an isometric imbedding of $\Sigma_{0}$ into $M$, and $\mathfrak{i}\left(\Sigma_{0}\right)$ is precisely the level set $\Sigma_{\tau_{0}}$ of $t$.

- The element $\Phi$ represents the matter field on $M$ : in the E-S setting, then $\Phi=\phi \in C^{\infty}(M)$, while in the E-M setting, we have $\Phi=F \in \Gamma \Lambda^{2} M$.

- Both the Einstein equations (18) and the appropriate field equations for $\Phi$ (either (20) or (22)) are satisfied on $(M, g)$.

- The field $k_{0}$ corresponds to the future second fundamental form of $\Sigma_{\tau_{0}}$. In other words, $k_{0}$ coincides with the pullback $\mathrm{i}^{*} k$.

- The field $\mathfrak{f}_{0}$ corresponds with the restriction of $\Phi$ to $\Sigma_{0}$. To be more precise, in the E-S setting, this means

$$
\phi_{0}=\mathfrak{i}^{*} \phi, \quad \phi_{1}=\mathfrak{i}^{*} \mathcal{L}_{t} \phi .
$$

In the E-M setting, if $E, H$ is the electromagnetic decomposition of $F$, then

$$
E_{0}=\mathfrak{i}^{*} E, \quad H_{0}=\mathfrak{i}^{*} H .
$$

If all the above conditions hold, then $(M, g, \Phi)$, along with $\mathfrak{i}$ and $t$, will be called a solution of the (Einstein-scalar or Einstein-Maxwell) CMC Cauchy problem corresponding to the initial data set $\left(\Sigma_{0}, \gamma_{0}, k_{0}, \mathfrak{f}_{0}\right)$. 
Our next task is to state a local well-posedness theorem for both of the above CMC Cauchy problems. This will be a straightforward modification of the vacuum analogue stated in [17, Prop. 6.1].

Theorem 7. Let $\left(\Sigma_{0}, \gamma_{0}, k_{0}, \mathfrak{f}_{0}\right)$ be an admissible initial data set for the $E$-S or $E-M$ equations, and let $\tau_{0}=\operatorname{tr} k_{0}<0$. Then, there exists a solution $(M, g, \Phi)$ of the E-S/E-M (resp.) CMC Cauchy problem corresponding to the above initial data set, with $(M, g)$ satisfying (CMC $)_{t, t_{0}, t_{1}}$ for some time function $t$ on $M$ and $t_{0}<\tau_{0}<t_{1}<0$. Furthermore, the solution is unique up to isometric imbedding, and the time of existence $t_{1}-\tau_{0}$ depends continuously on the following parameters:

- The initial mean curvature $\tau_{0}$.

- The diameter and injectivity radius of $\Sigma_{0}$.

- The following Sobolev norm for $k_{0}$ :

$$
\mathfrak{K}_{0}=\left\|k_{0}\right\|_{L^{4}\left(\Sigma_{0}\right)}+\left\|\nabla k_{0}\right\|_{L^{2}\left(\Sigma_{0}\right)}+\left\|\nabla^{2} k_{0}\right\|_{L^{2}\left(\Sigma_{0}\right)}+\left\|\nabla^{3} k_{0}\right\|_{L^{2}\left(\Sigma_{0}\right)} \text {. }
$$

- The following Sobolev norm for the curvature $\mathcal{R}_{0}$ of $\Sigma_{0}$ :

$$
\mathfrak{R}_{0}=\left\|\mathcal{R}_{0}\right\|_{L^{2}\left(\Sigma_{0}\right)}+\left\|\nabla \mathcal{R}_{0}\right\|_{L^{2}\left(\Sigma_{0}\right)}+\left\|\nabla^{2} \mathcal{R}_{0}\right\|_{L^{2}\left(\Sigma_{0}\right)} .
$$

- In the E-S case, the following Sobolev norm for $\mathfrak{f}_{0}=\left(\phi_{0}, \phi_{1}\right)$ :

$$
\begin{aligned}
\mathfrak{F}_{0}=\| & \nabla \phi_{0}\left\|_{L^{4}\left(\Sigma_{0}\right)}+\right\| \nabla^{2} \phi_{0}\left\|_{L^{2}\left(\Sigma_{0}\right)}+\right\| \nabla^{3} \phi_{0}\left\|_{L^{2}\left(\Sigma_{0}\right)}+\right\| \nabla^{4} \phi_{0} \|_{L^{2}(\Sigma)} \\
& +\left\|\phi_{1}\right\|_{L^{4}\left(\Sigma_{0}\right)}+\left\|\nabla \phi_{1}\right\|_{L^{2}\left(\Sigma_{0}\right)}+\left\|\nabla^{2} \phi_{1}\right\|_{L^{2}\left(\Sigma_{0}\right)}+\left\|\nabla^{3} \phi_{1}\right\|_{L^{2}\left(\Sigma_{0}\right)},
\end{aligned}
$$

- In the $E-M$ case, the following Sobolev norm for $\mathfrak{f}_{0}=\left(E_{0}, H_{0}\right)$ :

$$
\begin{aligned}
\mathfrak{F}_{0}=\| & E_{0}\left\|_{L^{4}\left(\Sigma_{0}\right)}+\right\| \nabla E_{0}\left\|_{L^{2}\left(\Sigma_{0}\right)}+\right\| \nabla^{2} E_{0}\left\|_{L^{2}\left(\Sigma_{0}\right)}+\right\| \nabla^{3} E_{0} \|_{L^{2}\left(\Sigma_{0}\right)} \\
& +\left\|H_{0}\right\|_{L^{4}\left(\Sigma_{0}\right)}+\left\|\nabla H_{0}\right\|_{L^{2}\left(\Sigma_{0}\right)}+\left\|\nabla^{2} H_{0}\right\|_{L^{2}\left(\Sigma_{0}\right)}+\left\|\nabla^{3} H_{0}\right\|_{L^{2}\left(\Sigma_{0}\right)} .
\end{aligned}
$$

The ideas behind the proof of Theorem 7 are standard. The main points of the proof are summarized in further detail in [21, Sec. 6.2].

Remark. Both Theorem 7 and [17, Prop. 6.1] are derived by solving the Einstein equations in the CMC gauge along with transported coordinate systems. This, however, is by no means an optimal result. For a local well-posedness result requiring less differentiability in the CMC gauge in the vacuum setting, see [2].

3.4. Outline of the Proof of Theorem 6. Like the other breakdown results discussed in the introduction, the proof of Theorem 6 is at its highest level intimately tied to the corresponding local well-posedness result of Theorem 7 Our strategy for proving Theorem [6 will be analogous to that of the model breakdown problem for the nonlinear wave equation (11). Indeed, we aim to bound the parameters on the timeslices in our spacetime which control the time of existence in Theorem 7 .

Assume now the hypotheses of Theorem [6 For each $t_{0}<\tau<t_{1}$, we define:

$$
\begin{aligned}
\mathfrak{K}(\tau) & =\|k\|_{L^{4}\left(\Sigma_{\tau}\right)}+\|\nabla k\|_{L^{2}\left(\Sigma_{\tau}\right)}+\left\|\nabla^{2} k\right\|_{L^{2}\left(\Sigma_{\tau}\right)}+\left\|\nabla^{3} k\right\|_{L^{2}\left(\Sigma_{\tau}\right)}, \\
\mathfrak{R}(\tau) & =\|\mathcal{R}\|_{L^{2}\left(\Sigma_{\tau}\right)}+\|\nabla \mathcal{R}\|_{L^{2}\left(\Sigma_{\tau}\right)}+\left\|\nabla^{2} \mathcal{R}\right\|_{L^{2}\left(\Sigma_{\tau}\right)},
\end{aligned}
$$

as well as a corresponding matter field energy $\mathfrak{f}(\tau)$. In the E-S case, we define

$$
\begin{gathered}
\mathfrak{f}(\tau)=\|\nabla \phi\|_{L^{4}\left(\Sigma_{\tau}\right)}+\left\|\nabla^{2} \phi\right\|_{L^{2}\left(\Sigma_{\tau}\right)}+\left\|\nabla^{3} \phi\right\|_{L^{2}\left(\Sigma_{\tau}\right)}+\left\|\nabla^{4} \phi\right\|_{L^{2}\left(\Sigma_{\tau}\right)} \\
+\left\|\mathcal{L}_{t} \phi\right\|_{L^{4}\left(\Sigma_{\tau}\right)}+\left\|\nabla\left(\mathcal{L}_{t} \phi\right)\right\|_{L^{2}\left(\Sigma_{\tau}\right)}
\end{gathered}
$$




$$
+\left\|\nabla^{2}\left(\mathcal{L}_{t} \phi\right)\right\|_{L^{2}\left(\Sigma_{\tau}\right)}+\left\|\nabla^{3}\left(\mathcal{L}_{t} \phi\right)\right\|_{L^{2}\left(\Sigma_{\tau}\right)},
$$

while in the E-M case, we define

$$
\begin{aligned}
\mathfrak{f}(\tau)=\|E\|_{L^{4}\left(\Sigma_{\tau}\right)}+\|\nabla E\|_{L^{2}\left(\Sigma_{\tau}\right)}+\left\|\nabla^{2} E\right\|_{L^{2}\left(\Sigma_{\tau}\right)}+\left\|\nabla^{3} E\right\|_{L^{2}\left(\Sigma_{\tau}\right)} \\
+\|H\|_{L^{4}\left(\Sigma_{\tau}\right)}+\|\nabla H\|_{L^{2}\left(\Sigma_{\tau}\right)}+\left\|\nabla^{2} H\right\|_{L^{2}\left(\Sigma_{\tau}\right)}+\left\|\nabla^{3} H\right\|_{L^{2}\left(\Sigma_{\tau}\right)},
\end{aligned}
$$

where $E, H \in \underline{\mathfrak{X}}^{*}(M)$ is the electromagnetic decomposition of $F$.

For convenience, we also fix an "initial time"

$$
\max \left(t_{0}, 2 t_{1}\right)<\tau_{0}<t_{1},
$$

and we treat $\Sigma_{\tau_{0}}$ as the "initial timeslice" in $M$. From now on, we will only be concerned with the timespan $\left[\tau_{0}, t_{1}\right)$ in this proof. For convenience, we also define

$$
M_{+}=\Sigma_{\left[\tau_{0}, t_{1}\right)}=\left\{q \in M \mid \tau_{0} \leq t(q)<t_{1}\right\} .
$$

The mean curvatures of the $\Sigma_{\tau}$ 's, $\tau_{0} \leq \tau<t_{1}$, are trivially comparable to $\left|t_{1}\right|$. Suppose we can also uniformly control the following:

- The diameters and the injectivty radii of the $\Sigma_{\tau}$ 's, $\tau_{0} \leq \tau<t_{1}$.

- The quantities $\mathfrak{K}(\tau), \mathfrak{R}(\tau)$, and $\mathfrak{f}(\tau)$ for all $\tau_{0} \leq \tau<t_{1}$.

Then, applying Theorem 7 to each of the timeslices of $M$, we obtain roughly that there exists some sufficiently small $0<\epsilon<\left|t_{1}\right|$ such that for every $\tau_{0} \leq \tau<t_{1}$ :

- A solution to the Cauchy problem exists with initial data given by $\Sigma_{\tau}$.

- The solution exists on a time interval including $[\tau, \tau+\epsilon)$.

- The solution is unique up to isometric imbedding.

By combining the above solutions, we obtain the desired continuation of $(M, g, \Phi)$ to the time interval $\left(t_{0}, t_{1}+\epsilon\right)$, which completes the proof of Theorem 6 .

As a result, the main objectives will be that of controlling the diameters, the injectivity radii, and the energy quantities $\mathfrak{K}(\tau), \mathfrak{R}(\tau), \mathfrak{f}(\tau)$. The remainder of this paper will be dedicated to establishing these estimates. Of these, the energy bounds will be the primary task. The diameter and injectivity radius bounds, on the other hand, will be trivial consequences by the end of the proof.

Rather than directly controlling the quantities associated with $\mathfrak{K}(\tau), \mathfrak{R}(\tau)$, and $\mathfrak{f}(\tau)$, which are horizontal fields on the timeslices, we instead control norms of corresponding spacetime quantities on these timeslices. These include the spacetime Riemann curvature $R$ and the spacetime matter field, i.e., either $\phi$ or $F$. The necessary estimates for $\mathfrak{K}(\tau), \mathfrak{R}(\tau)$, and $\mathfrak{f}(\tau)$ can then be derived using standard elliptic estimates. For example, $R$ can be related to $\mathcal{R}$ and $k$ using the Gauss equations (32) and the Codazzi equations (33).

To control $R$ and the matter field $\Phi$, we apply variations of standard energymomentum tensor techniques. In particular, we take advantage of the observations that both $R$ and $\Phi$ satisfy covariant tensorial wave and Maxwell-type equations. We then construct "generalized energy-momentum tensors" based on these relations, and we apply these in the standard fanshion to derive basic energy inequalities.

The above suffices for "lower-order" a priori energy estimates. In order to derive "higher-order" energy estimates, however, we will also need uniform bounds for $R$ and for quantities derived from $\Phi$. 12 Since these quantities satisfy a system of covariant tensorial wave equations, we can then apply the representation formula presented in [22, Thm. 7] (and also in [21, Thm. 5.1]).

\footnotetext{
${ }^{12}$ More specifically, $D^{2} \phi$ in the E-S case, and $D F$ in the E-M case.
} 
The representation formula mentioned above is only valid on the "regular" portion of past null cones, i.e., prior to the null injectivity radius. Thus, in order to obtain satisfactory estimates using this formula, we must also control the geometry of past null cones. In particular, we must control the null injectivity radius and various connection quantities on these cones by other quantities which can be controlled a priori. In our case, these include $L^{2}$ "flux" quantities for $R$ and $\Phi$ on these cones, and other a priori bounds relating to the time foliation.

Unfortunately, this task is exceedingly difficult and is responsible for a vast portion of the technical work behind Theorem [6. For instance, this involves constructing a geometric tensorial Littlewood-Paley theory, cf. [13] and [21, Sec. 2.2], and applying it in a massive bootstrap argument. Within this argument are various Besov estimates as well as an elaborate sharp trace estimate for regular null cones. As a result, we omit a majority of this development from this paper. For details regarding this portion of the argument, see [21. 13

Using the above control on the local null geometry along with the representation formula of [22], we can derive all the necessary estimates for $R$ and $\Phi$. As a result, we can then estimate $\mathfrak{K}(\tau), \mathfrak{R}(\tau)$, and $\mathfrak{f}(\tau)$, as mentioned before, and hence Theorem 6 is proved. In the remaining sections of the paper, we will provide more detailed discussions on the steps described in this outline.

\section{Regular Past Null Cones}

As mentioned before, both the local energy estimates in this paper and the representation formula for covariant tensor wave equations in [22] are essential components of the proof of Theorem 6. Moreover, both depend heavily on the local null geometry of $(M, g)$. More specifically, both are applicable to our setting only on "regular" past null cones, where the null exponential map remains a diffeomorphism and its image retains a smooth structure.

We shall provide in this section some preliminaries on such regular past null cones. We mainly follow the development given in [21, Ch. 3] and [22, but we restrict ourselves in this paper to the special case of time foliated null cones. 14 This eliminates much of the minor technical irritations present in [21, 22] that arose from dealing with more general foliating functions.

4.1. Regular Past Null Cones. Assume the spacetime $(M, g, \Phi)$, along with the time foliation of $M$ given by the time function $t$, as expressed in the statement of Theorem 6. Fix $p \in M$, and consider the null exponential map $\exp _{p}$ about $p$, i.e., the exponential map about $p$ restricted to the past null cone $\mathfrak{N}$ of the tangent space $T_{p} M .15$ Define the past null cone $N^{-}(p)$ of $p$ to be the image of $\exp _{p}$.

By definition, $N^{-}(p)$ is ruled by the past inextendible null geodesics beginning at $p$. Recall that $\exp _{p}$ is a diffeomorphism between a sufficiently small neighborhood of 0 in $\mathfrak{N}$ and its image, which is then a smooth null hypersurface of $M$. We refer to such regions of $N^{-}(p)$ as "regular". A loss of such regularity can occur at a terminal point $z$ of $N^{-}(p)$, where one of the following scenarios hold:

- The point $z$ is a cut locus point, that is, distinct past null geodesics from $p$ intersect at $z$. In other words, the map $\exp _{p}$ fails to be one-to-one at $z$.

\footnotetext{
${ }^{13}$ Earlier work in this area for the vacuum case were done in $12,13,14,16,19,24,25$.

${ }^{14}$ This case was discussed in [21] Ch. 4].

${ }^{15}$ For convenience, we assume $\mathfrak{N}$ does not include the origin of $T_{p} M$.
} 
- The pair $p$ and $z$ are past null conjugate points. In other words, the map $\underline{\exp }_{p}$ fails to be nonsingular at $z$.

Define the function $t_{p}$ on $N^{-}(p)$ by $t_{p}(q)=t(p)-t(q)$, i.e., the difference in time between the vertex $p$ and the given point $q$. We also define the following:

- Let $\mathfrak{s}(p)$ be the infimum of all values $t_{p}(q)$ for which $q \in N^{-}(p)$ is a past null conjugate point. We call $\mathfrak{s}(p)$ the past null conjugacy radius of $p$ (with respect to the $t$-foliation). In the case that $N^{-}(p)$ has no conjugate points, we define $\mathfrak{s}(p)=t(p)-t_{0}$. Note that $\mathfrak{s}(p)$ indicates the largest $v>0$ such that $N^{-}(p)$ encounters no conjugate points before $t_{p}$-value $v$.

- Let $\mathfrak{l}(p)$ be the infimum of all values $t_{p}(q)$ for which $q \in N^{-}(p)$ is a cut locus point. On the other hand, if $N^{-}(p)$ has no cut locus points, we define $\mathfrak{l}(p)=t(p)-t_{0}$. Then, $\mathfrak{l}(p)$ indicates the largest $v>0$ such that $N^{-}(p)$ encounters no cut locus points before $t_{p}$-value $v$.

- Define $\mathfrak{i}(p)=\min (\mathfrak{s}(p), \mathfrak{l}(p))$. We call this the past null injectivity radius of $p$ (with respect to the $t$-foliation).

We will refer to the region

$$
\mathcal{N}^{-}(p)=\left\{q \in N^{-}(p) \mid t_{p}(q)<\mathfrak{i}(p)\right\}
$$

as the regular past null cone of $p$. Then, the null exponential map $\exp _{p}$ is a diffeomorphism between a neighborhood in $\mathfrak{N}$ and $\mathcal{N}^{-}(p)$, and $\mathcal{N}^{-}(p)$ is indeed a smooth null hypersurface of $M$. From now on, we will only refer to the regular null cone $\mathcal{N}^{-}(p)$, as $N^{-}(p)$ is in general too irregular for our use.

In addition, for any $0<v \leq \mathfrak{i}(p)$, we define the null cone segment

$$
\mathcal{N}^{-}(p ; v)=\left\{q \in \mathcal{N}^{-}(p) \mid t_{p}(q)<v\right\},
$$

4.2. Normalization and Foliation. Since tangent null vectors in $\mathcal{N}^{-}(p)$ have vanishing Lorentzian "length" and are orthogonal to $\mathcal{N}^{-}(p)$, they cannot be normalized without introducing vectors transversal to $\mathcal{N}^{-}(p)$. Consequently, in our treatment, we will require an additional choice of a future timelike unit vector $\mathfrak{t} \in T_{p} M$ at $p$. For this, we use the most natural choice based on the problem at hand: the value of the future unit normal $T$ to the $\Sigma_{\tau}$ 's at $p$.

We define the null generators of $\mathcal{N}^{-}(p)$ (or of $N^{-}(p)$ ) to be the inextendible past null geodesics $\gamma$ on $M$ which satisfy $\gamma(0)=p$ and $g\left(\gamma^{\prime}(0),\left.T\right|_{p}\right)=1$. We can smoothly parametrize these generators by $\mathbb{S}^{2}$ using the following process. If we choose an orthonormal basis $e_{0}, \ldots, e_{3}$ of $T_{p} M$, with $e_{0}=\left.T\right|_{p}$, then we can identify each $\omega \in \mathbb{S}^{2}$ with the null generator $\gamma_{\omega}$ satisfying $\gamma_{\omega}^{\prime}(0)=-e_{0}+\omega^{k} e_{k}$. For convenience, we assume such a parametrization of the null generators of $\mathcal{N}^{-}(p)$, and we denote by $\gamma_{\omega}$ the null generator corresponding to $\omega$.

Remark. The objects on $\mathcal{N}^{-}(p)$ that we will discuss are of course defined independently of any parametrization of the null generators. However, for ease of notation, we will work explicitly with $\mathbb{S}^{2}$.

In addition, we define $L \in \mathfrak{X}\left(\mathcal{N}^{-}(p)\right)$ to be the tangent vector fields of the null generators of $\mathcal{N}^{-}(p)$, i.e., we define $\left.L\right|_{\gamma_{\omega}(v)}=\gamma_{\omega}^{\prime}(v)$ for any $\omega \in \mathbb{S}^{2}$ and $0<v<\mathfrak{i}(p)$. We note in particular that $L$ is a geodesic vector field.

As in previous works, e.g., [12, 19, 21, 22, 24, we would like to express $\mathcal{N}^{-}(p)$ as a foliation of spherical cross-sections. This was done abstractly in [21, Sec. 3.1] and 22 , Sec. 2] for the sake of the generalized representation formula for wave 
equations. Here, we can simplify our presentation by considering only the special case of the foliating function $t_{p}$, the most natural choice for the current setting.

Define the null lapse function $\vartheta$ by the formula

$$
\vartheta=\left(L t_{p}\right)^{-1} \in C^{\infty}\left(\mathcal{N}^{-}(p)\right) \text {. }
$$

Note that $\vartheta$ is everywhere strictly positive, and $\vartheta$ satisfies the initial limits

$$
\left.\lim _{v \searrow 0} \vartheta\right|_{\gamma_{\omega}(v)}=n(p) .
$$

The positivity of $\vartheta$ implies $d t_{p}$ is nonvanishing, so the level sets of $t_{p}$, denoted

$$
\mathcal{S}_{v}=\left\{q \in \mathcal{N}^{-}(p) \mid t_{p}(q)=v\right\}, \quad v>0,
$$

form a family of hypersurfaces of $\mathcal{N}^{-}(p)$. Since $L$ represents the unique null direction tangent to $\mathcal{N}^{-}(p)$, and the positivity of $\vartheta$ implies $L$ is transverse to each $\mathcal{S}_{v}$, we can conclude that each $\mathcal{S}_{v}$ is spacelike, i.e., Riemannian. Furthermore, the definition of $\mathfrak{i}(p)$ implies $\mathcal{S}_{v}$ is diffeomorphic to $\mathbb{S}^{2}$ for every $0<v<\mathfrak{i}(p)$.

We adopt the conventions of Section 2.2 to discuss this foliation of $\mathcal{N}^{-}(p)$, with $N=\mathcal{N}^{-}(p)$ and $f=t_{p}$. In order to distinguish this from the time foliation of $M$, we denote the induced horizontal metrics and connections on the $\mathcal{S}_{v}$ 's by $\lambda$ and $\not$, respectively. Mixed connections on $\mathcal{N}^{-}(p)$ are denoted by $\bar{\nabla}$, and the Gauss curvatures of the $\mathcal{S}_{v}$ 's are denoted by $\mathcal{K} \in C^{\infty}\left(\mathcal{N}^{-}(p)\right)$. In addition, the "normalized normal" vector field in this case is given by $Z=\vartheta L$, i.e., $\bar{\nabla}_{t_{p}}=\vartheta \bar{\nabla}_{L}$.

Since $\mathcal{N}^{-}(p)$ is null, we have no volume form on $\mathcal{N}^{-}(p)$ with respect to which we can integrate scalar functions. However, we can still provide a canonical definition for integrals of functions over $\mathcal{N}^{-}(p)$. Indeed, we define this integral by

$$
\int_{\mathcal{N}^{-}(p)} \phi=\int_{0}^{\infty}\left(\int_{\mathcal{S}_{v}} \vartheta \cdot \phi\right) d v
$$

for any $\phi \in C^{\infty}\left(\mathcal{N}^{-}(p)\right)$ for which the right-hand side is well-defined. We can similarly define integrals over any open subset of $\mathcal{N}^{-}(p)$, in particular for $\mathcal{N}^{-}(p ; v)$.

Remark. We can show using the change of variables formula that (50) is in fact independent of the foliating function of $\mathcal{N}^{-}(p)$; see [21, Prop. 3.4]. Later, we will further justify (50) in terms of general local energy estimates.

4.3. Parametrizations and Null Frames. We can parametrize $\mathcal{N}^{-}(p)$ using $t_{p}$ and a spherical value. For any $0<v<\mathfrak{i}(p)$ and $\omega \in \mathbb{S}^{2}$, we can identify the pair $(v, \omega)$ with the unique point $q$ on both the null generator $\gamma_{\omega}$ and $\mathcal{S}_{v}$. As a result, we can naturally treat any $\phi \in C^{\infty}\left(\mathcal{N}^{-}(p)\right)$ as a smooth function on the cylinder $(0, \mathfrak{i}(p)) \times \mathbb{S}^{2}$. For any such $\phi$, we denote by $\left.\phi\right|_{(v, \omega)}$ the value of $\phi$ at the point $q$ corresponding to the parameters $(v, \omega)$. We will freely use this $(v, \omega)$-notation throughout future sections without further elaboration.

In general, null frames are local frames $\hat{l}, \hat{m}, e_{1}, e_{2}$ which satisfy

$$
\begin{aligned}
g(\hat{l}, \hat{l}) & =g(\hat{m}, \hat{m}) \equiv 0, & g(\hat{l}, \hat{m}) \equiv-2, \\
g\left(\hat{l}, e_{a}\right) & =g\left(\hat{m}, e_{a}\right) \equiv 0, & g\left(e_{a}, e_{b}\right)=\delta_{a b},
\end{aligned}
$$

Here, we construct null frames which are adapted to our $t_{p}$-foliation of $\mathcal{N}^{-}(p)$.

Each point of $\mathcal{S}_{v}$ is normal to exactly two null directions, one of which is represented by $L$. We define $\underline{L} \in \overline{\mathfrak{X}}\left(\mathcal{N}^{-}(p)\right)$, called the conjugate null vector field, to 
be the vector field in the other normal null direction, subject to the normalization $g(L, \underline{L}) \equiv-2$. A direct calculation yields the following explicit formula for $\underline{L}$ :

$$
\underline{L}=-n^{-2} \vartheta^{2} L-2 n^{-1} \vartheta T \text {. }
$$

Next, we append to $L$ and $\underline{L}$ a local orthonormal frame $e_{1}, e_{2}$ on the $\mathcal{S}_{v}$ 's. Then, $\left\{L, \underline{L}, e_{1}, e_{2}\right\}$ defines a natural null frame for $\mathcal{N}^{-}(p)$. We adopt the following indexing conventions for adapted null frames.

- Horizontal indices 1,2 correspond to the directions $e_{1}$ and $e_{2}$.

- $\underline{L}$ corresponds to the index 3 , while $L$ corresponds to the index 4 .

Next, if we define the vector field

$$
N=\frac{1}{2}\left(n^{-1} \vartheta L-n \vartheta^{-1} \underline{L}\right) \in \overline{\mathfrak{X}}\left(\mathcal{N}^{-}(p)\right),
$$

then (51) implies $g(N, T) \equiv 0$ and $g(N, N) \equiv 1$. In other words, $N$ is the outer unit normal to each $\mathcal{S}_{v}$ in $\Sigma_{t(p)-v}$. Using (51) again, we can derive the following:

$$
L=-n \vartheta^{-1}(T-N), \quad \underline{L}=-n^{-1} \vartheta(T+N) .
$$

With this, we can compute the $(h-)$ norms of $L$ and $\underline{L}$,

$$
|L|^{2}=2 n^{2} \vartheta^{-2}, \quad|\underline{L}|^{2}=2 n^{-2} \vartheta^{2} .
$$

4.4. Ricci Coefficients. We will make use of the following connection quantities:

- Define the null second fundamental forms $\chi, \underline{\chi} \in \Gamma \underline{T}^{2} \mathcal{N}^{-}(p)$ by

$$
\chi(X, Y)=g\left(\bar{D}_{X} L, Y\right), \quad \underline{\chi}(X, Y)=g\left(\bar{D}_{X} \underline{L}, Y\right), \quad X, Y \in \underline{\mathfrak{X}}\left(\mathcal{N}^{-}(p)\right) .
$$

Both $\chi$ and $\underline{\chi}$ are symmetric, since both $L$ and $\underline{L}$ are normal to the $\mathcal{S}_{v}$ 's. We often decompose $\chi$ into its trace and traceless parts:

$$
\operatorname{tr} \chi=\lambda^{a b} \chi_{a b}, \quad \hat{\chi}=\chi-\frac{1}{2}(\operatorname{tr} \chi) \lambda .
$$

We also use an analogous decomposition for $\underline{\chi}$.

- Define $\zeta, \underline{\eta} \in \Gamma \underline{T}^{1} \mathcal{N}^{-}(p)$ by

$$
\zeta(X)=\frac{1}{2} g\left(\bar{D}_{X} L, \underline{L}\right), \quad \underline{\eta}(X)=\frac{1}{2} g\left(X, \bar{D}_{L} \underline{L}\right), \quad X \in \underline{\mathfrak{X}}\left(\mathcal{N}^{-}(p)\right) \text {. }
$$

From [12, Prop. 2.7], we have the following relation between $\zeta$ and $\eta$ :

$$
\underline{\eta}=-\zeta+\not \nabla(\log \vartheta) \text {. }
$$

The quantities $\operatorname{tr} \chi, \hat{\chi}$, and $\zeta$ are called the expansion, shear, and torsion of $\mathcal{N}^{-}(p)$. We refer to the collection of fields $\operatorname{tr} \chi, \hat{\chi}, \operatorname{tr} \underline{\chi}, \underline{\hat{\chi}}, \zeta, \underline{\eta}$ as the Ricci coefficients of $\mathcal{N}^{-}(p)$ (with respect to the $t_{p}$-foliation).

From (51) and direct calculations, we see that $\underline{\chi}$ and $\eta$ are intimately tied to the time foliation of $M$ via the following formulas:

$$
\begin{aligned}
\underline{\eta}_{a} & =k_{i a} N^{i}+\not_{a}(\log n), \\
\underline{\chi}_{a b} & =-n^{-2} \vartheta^{2} \cdot \chi_{a b}+2 n^{-1} \vartheta \cdot k_{a b} .
\end{aligned}
$$

Lastly, we define the mass aspect function $\mu \in C^{\infty}\left(\mathcal{N}^{-}(p)\right)$ by

$$
\mu=\not^{a} \zeta_{a}-\frac{1}{2} \hat{\chi}^{a b} \underline{\hat{\chi}}_{a b}+|\zeta|^{2}+\frac{1}{4} R_{4343}-\frac{1}{2} R_{43} .
$$

This quantity is present in the representation formula for wave equations, and it plays a crucial role in controlling the local past null geometry. 
4.5. Initial Values. We now briefly examine the initial values of various quantities on $\mathcal{N}^{-}(p)$, that is, we look at the limits of these quantities at $p$ along null generators. This is of importance in the problem of controlling the local null geometry, since our goal will be to show that these values differ little from the initial value in some specific sense. Moreover, these initial value computations were applied in the derivations of the representation formulas of [15, 22].

We opt in this paper to skip the technical details on this topic, since most of them are rather distant from the heart of Theorem 66. For instance, some of the derivations require applications of convex geometry and bitensor fields. A complete exposition in the case of general foliating functions can be found in [21, Sec. 3.3]; the case of the $t_{p}$-foliation is covered in [21, Sec. 4.2]. An earlier account for the geodesic foliation case, which is needed for the general case, is presented in [24.

The first task is to examine the horizontal metrics $\lambda$ at the initial limit. Note that a coordinate system $(U, \varphi)$ in $\mathbb{S}^{2}$ generates a transported coordinate system on each $\mathcal{S}_{v}$ by mapping the point with parameters $(v, \omega)$ to $\varphi(\omega)$.

Proposition 8. Let $\lambda_{0}$ denote the Euclidean metric on $\mathbb{S}^{2}$, and fix a coordinate system $(U, \varphi)$ on $\mathbb{S}^{2}$. Index $\lambda_{0}$ using $\varphi$-coordinates, and index $\lambda$ on each $\mathcal{S}_{v}$ using the associated transported coordinate system. Then, for any $\omega \in U$,

$$
\left.\lim _{v \searrow 0} v^{-2} \lambda_{i j}\right|_{(v, \omega)}=\left.\left.n^{2}\right|_{p} \cdot\left(\lambda_{0}\right)_{i j}\right|_{\omega}, \quad 1 \leq i, j \leq 2 .
$$

Proof. The proof involves relating the " $(v, \omega)$-parametrization" of $\mathcal{N}^{-}(p)$ with normal coordinates; see [21, Prop. 3.15].

Proposition 8 implies the following integral limit:

Corollary 9. Let $\phi \in C^{\infty}\left(\mathcal{N}^{-}(p)\right)$, let $\phi_{0} \in C^{\infty}\left(\mathbb{S}^{2}\right)$, and suppose

$$
\left.\lim _{v \searrow 0} \phi\right|_{(v, \omega)}=\phi_{0}(\omega), \quad \omega \in \mathbb{S}^{2} .
$$

Then, the following integral limit holds:

$$
\lim _{v \searrow 0} v^{-2} \int_{\mathcal{S}_{v}} \phi=\left.n^{2}\right|_{p} \cdot \int_{\mathbb{S}^{2}} \phi_{0}
$$

The next proposition deals with the effects of derivatives on initial limits:

Proposition 10. Let $A \in \Gamma \underline{\mathcal{T}} \mathcal{N}^{-}(p)$, and suppose

$$
\lim _{v \searrow 0}|A|_{(v, \omega)}=0, \quad \omega \in \mathbb{S}^{2} .
$$

Then, for any integer $k>0$, we also have

$$
\lim _{v \searrow 0} v^{k} \mid \nabla^{k} A \|_{(v, \omega)}=0, \quad \omega \in \mathbb{S}^{2} .
$$

Proof. See [21, Prop. 3.17].

Finally, we consider the Ricci coefficients:

Proposition 11. The following limits hold for each $\omega \in \mathbb{S}^{2}$.

- We have the following limits for $\chi$ :

$$
\lim _{v \searrow 0}\left|\vartheta(\operatorname{tr} \chi)-2 t_{p}^{-1}\left\|_{(v, \omega)}=0, \quad \lim _{v \searrow 0} \mid \hat{\chi}\right\|_{(v, \omega)}=0 .\right.
$$


- We have the following limits for $\zeta$ and $\underline{\eta}$ :

$$
\lim _{v \searrow 0}|\zeta|_{(v, \omega)}=\lim _{v \searrow 0}|\underline{\eta}|_{(v, \omega)} \lesssim|\pi|_{p} .
$$

Proof. Equation (58) is an immediate consequence of [21, Prop. 3.18]. The limit for $\underline{\eta}$ in (59) is an immediate consequence of (55), while the derivation for $\zeta$ in (59) can be found in [21, Prop. 4.9].

Remark. We can also obtain analogous initial value properties for both $\underline{\chi}$ and $\mu$. For details, see [21, Sec. 3.3, Sec. 4.2].

\section{A Priori Estimates}

In this section, we derive a priori estimates which are consequences of the breakdown criterion (40). These are fundamental to the proof of Theorem 6 since they determine the nature of the null geometry estimates and the higher-order estimates which we must prove. In other words, we must control the null geometry and the higher-order energy norms by quantities which can be controlled a priori.

We now introduce the notion of "fundamental constants", i.e., values on which all of our "universal" constants will depend. More explicitly, whenever we write $A \lesssim B$, we mean $A \leq C B$ for some constant $C$ depending only on these fundamental constants. Similarly, if we write $A \simeq B$, then we mean $C^{-1} B \leq A \leq C B$ for some constant $C$ depending only on these fundamental constants.

The complete list of such fundamental constants is given below:

- The "breakdown time" $t_{1}$.

- The "breakdown criterion" constant $C_{0}$ in (40).

- The intrinsic and extrinsic geometries of the "initial timeslice" $\Sigma_{\tau_{0}}$, and the values of the fields defined on $\Sigma_{\tau_{0}}$. These include derivatives of $R, \Phi, k, n$, etc., restricted to $\Sigma_{\tau_{0}}$, as well as the volume $V\left(\Sigma_{\tau_{0}}\right)$ of $\tau_{0}$.

Remark. We can be more explicit on the exact properties of $\Sigma_{\tau_{0}}$ and values on $\Sigma_{\tau_{0}}$ for this dependence; see [21, Sec. 6.3]. However, the main idea is that we can uniformly control a sufficient amount of quantities (as dictated in Section 3.4) on all the $\Sigma_{\tau}$ 's, $\tau_{0} \leq \tau<t_{1}$, by properties of only $\Sigma_{\tau_{0}}$.

5.1. Regularity of the Time Foliation. Our first task will be to establish some basic control for our time foliation. From the breakdown criterion (40) along with (19), (21), (23), (38), we can immediately obtain the following:

$$
\|\pi\|_{L^{\infty}(M)} \lesssim 1, \quad\|Q\|_{L^{\infty}(M)}+\|\operatorname{Ric}\|_{L^{\infty}(M)} \lesssim 1 .
$$

Here, $\pi$ is the deformation tensor of $T$, while $Q$ and Ric denote the energymomentum tensor of the matter field and the Ricci curvature of $(M, g)$.

Contracting (35) and recalling the $\mathrm{CMC}$ gauge condition yields the lapse equation

$$
\Delta n=n\left[|k|^{2}+\operatorname{Ric}(T, T)\right]-1 .
$$

Moreover, the strong energy condition implies that $\operatorname{Ric}(T, T) \geq 0$ in both the E-S and E-M settings. As a result, we can derive the following bounds:

Proposition 12. On each $\Sigma_{\tau}, \tau_{0} \leq \tau<t_{1}$, we have the comparisons

$$
|n| \simeq 1, \quad V\left(\Sigma_{\tau}\right) \simeq 1
$$


Proof. At a minimum point $p$ of $n$ on $\Sigma_{\tau}$, we have from (61) that

$$
\left.n\left[\operatorname{Ric}(T, T)+|k|^{2}\right]\right|_{p} \geq 1
$$

By (60), we obtain a lower bound for $n$ depending only on the fundamental constants. Next, at a maximum point $p$ of $n$ on $\Sigma_{\tau}$, we have from (61) that

$$
n(p) \leq\left[\operatorname{Ric}(T, T)+|\hat{k}|^{2}+\frac{\tau^{2}}{3}\right]^{-1} \leq \frac{3}{\tau^{2}} \lesssim 1,
$$

where we also applied the strong energy condition on $\operatorname{Ric}(T, T)$.

For a volume form $V_{\tau}$ on $\Sigma_{\tau}$, we have

$$
\mathcal{L}_{t} V_{\tau}=-n(\operatorname{tr} k) V_{\tau}=-n t \cdot V_{\tau} \geq 0 .
$$

By the above explicit bound $n \leq 3 t^{-2}$, then

$$
0 \leq \frac{d}{d \tau} V\left(\Sigma_{\tau}\right)=-\tau \int_{\Sigma_{\tau}} n \leq-3 \tau^{-1} V(\tau) .
$$

From Grönwall's inequality, we obtain $V(\tau) \simeq \tau^{-3} \simeq 1$, as desired.

Furthermore, from (40) and Proposition 12, we have proved the uniform bounds

$$
\|n\|_{L^{\infty}\left(M_{+}\right)}+\left\|n^{-1}\right\|_{L^{\infty}\left(M_{+}\right)}+\|\nabla n\|_{L^{\infty}\left(M_{+}\right)}+\|k\|_{L^{\infty}\left(M_{+}\right)} \lesssim 1,
$$

where $M_{+}$is as defined in (47).

Next, we examine some coordinate regularity properties satisfied by the timeslices. To begin with, since $\Sigma_{\tau_{0}}$ is compact, we can find a constant $C>1$ and a finite covering of $\Sigma_{\tau_{0}}$ by local coordinate systems $\left(U_{1}, \varphi_{1}\right), \ldots,\left(U_{m}, \varphi_{m}\right)$ such that for each $1 \leq i \leq m$, we have the uniform ellipticity bound

$$
C^{-1}|\xi|^{2} \leq\left.\gamma_{j l}\right|_{p} \xi^{j} \xi^{l} \leq C|\xi|^{2}, \quad p \in U_{i}, \xi \in \mathbb{R}^{3},
$$

where $\gamma$ is indexed with respect to the $\varphi_{i}$-coordinates. We next show that this uniform ellipticity condition holds uniformly for all $\Sigma_{\tau}$ 's, $\tau_{0} \leq \tau<\tau_{1}$.

Proposition 13. Let $\left(U_{i}, \varphi_{i}\right), 1 \leq i \leq m$, be as given above, and, for each $\tau$, we let $\left(U_{i}^{\tau}, \varphi_{i}^{\tau}\right)$ denote the coordinate system on $\Sigma_{\tau}$ obtained by transporting $\left(U_{i}, \varphi_{i}\right)$. Then, there exists a constant $C_{b}>1$, depending on the fundamental constants, such that for any $\tau_{0} \leq \tau<t_{1}$ and $1 \leq i \leq m$, we have the estimates

$$
C_{\mathrm{b}}^{-1}|\xi|^{2} \leq\left.\gamma_{j l}\right|_{p} \xi^{j} \xi^{l} \leq C_{\mathrm{b}}|\xi|^{2}, \quad p \in U_{i}^{\tau}, \xi \in \mathbb{R}^{3},
$$

where $\gamma$ is indexed with respect to the $\varphi_{i}^{\tau}$-coordinates. In other words, the uniform ellipticity condition (64) holds uniformly on all the $\Sigma_{\tau}$ 's.

We sketch the proof of Proposition 13 below. For further details, we refer the reader to [21, Prop. 4.6], as well as [16, Prop. 4.1] and [17, Prop. 2.4].

Proof. Fix a coordinate system $(U, \varphi)=\left(U_{i}, \varphi_{i}\right)$ of $\Sigma_{\tau_{0}}$, and consider its transported systems $\left(U^{\tau}, \varphi^{\tau}\right)=\left(U_{i}^{\tau}, \varphi_{i}^{\tau}\right)$; we will index with respect to these coordinates. Fix $\xi \in \mathbb{R}^{3}$, and let $X=\xi^{i} \partial_{i}$. By (34), we have $\mathcal{L}_{t}|X|^{2}=-2 n \cdot k(X, X)$, so

$$
-2\|n\|_{L^{\infty}\left(\Sigma_{\tau}\right)}\|k\|_{L^{\infty}\left(\Sigma_{\tau}\right)}|X|^{2} \leq \mathcal{L}_{t}|X|^{2} \leq 2\|n\|_{L^{\infty}\left(\Sigma_{\tau}\right)}\|k\|_{L^{\infty}\left(\Sigma_{\tau}\right)}|X|^{2} .
$$

Integrating (66) along the integral curves of $Z=n T$ (or equivalently, of $T$ ) and applying Grönwall's inequality, we obtain for each $p \in U$ the comparisons

$$
\left.\bar{C}^{-1}|X|^{2}\right|_{p} \leq\left.|X|^{2}\right|_{p^{\tau}} \leq\left.\bar{C}|X|^{2}\right|_{p}, \quad \tau \in \mathcal{I}
$$


where $p^{\tau}$ is the normal transport of $p$ along $Z$ to $\Sigma^{\tau}$, and where

$$
\bar{C}=\exp \left[2\left(t_{1}-\tau_{0}\right)\|n\|_{L^{\infty}\left(M_{+}\right)}\|\pi\|_{L^{\infty}\left(M_{+}\right)}\right] \lesssim 1 .
$$

Defining $C_{b}=C \bar{C}$, where $C$ is as in (64), varying over all $\xi \in \mathbb{R}^{3}, 1 \leq i \leq m$, and $p \in \Sigma_{\tau_{0}}$, and recalling the bound (64), then we obtain (65), as desired.

By considering the family of transported coordinate systems from Proposition [13. we can deduce some rudimentary Riemannian geometric estimates on the $\Sigma_{\tau}$ 's. For a point $x \in \mathbb{R}^{3}$ and $r>0$, we let $\mathcal{B}(x, r)$ denote the Euclidean ball in $\mathbb{R}^{3}$ about $x$ of radius $r$. The property given in the subsequent proposition is an immediate consequence of Proposition 13 and the compactness of the $\Sigma_{\tau}$ 's.

Proposition 14. Let $\left(U_{i}^{\tau}, \varphi_{i}^{\tau}\right)$ be defined as in Proposition 13, for all $\tau_{0} \leq \tau<t_{1}$ and $1 \leq i \leq m$. There exists $r_{0}>0$, depending on the fundamental constants, such that for any $\tau_{0} \leq \tau<t_{1}$ and $p \in \Sigma_{\tau}$, there exists $1 \leq i \leq m$ such that $p \in U_{i}^{\tau}$, and

$$
\varphi_{i}^{\tau}\left(U_{i}^{\tau}\right) \supseteq \mathcal{B}\left(\varphi_{i}^{\tau}(p), r_{0}\right) .
$$

Proof. See [16, Lemma 2.2] and [21, Prop. 4.6].

Next, for any $\tau_{0} \leq \tau<t_{1}, p \in \Sigma_{\tau}$, and $\rho>0$, we let $B_{\tau}(p, \rho)$ denote the geodesic ball in $\Sigma_{\tau}$ of radius $\rho$ about $p$. In addition, for $\tau$ and $p$ as above, we define

$$
r_{\tau}(p)=\inf _{\sigma \leq 1} \frac{V\left(B_{\tau}(p, \sigma)\right)}{\sigma^{3}},
$$

i.e., the volume radius at $p$ (in $\Sigma_{\tau}$ ) with scale 1 . By considering the transported coordinate systems in the statement of Proposition 13, we can derive the following uniform lower bound for the above volume radii.

Proposition 15. There exists $\rho>0$, depending on the fundamental constants, such that $r_{\tau}(p) \geq \rho$ for every $\tau_{0} \leq \tau<t_{1}$ and $p \in \Sigma_{\tau}$.

Proof. See the proof of 21, Cor. 2.1]. The main idea is that the uniform ellipticity condition (64) implies that geodesic balls are comparable to Euclidean balls.

5.2. Sobolev Inequalities. Proposition 13 also plays an instrumental role in deriving uniform Sobolev estimates on the $\Sigma_{\tau}$ 's. Indeed, we can derive such first-order Sobolev inequalities on each $\Sigma_{\tau}$ by using a partition of unity argument and applying the corresponding Euclidean Sobolev inequality to each coordinate system $\left(U_{i}^{\tau}, \varphi_{i}^{\tau}\right)$. As a result of this, we obtain the following scalar Sobolev inequalities:

Lemma 16. The following hold for any $f \in C^{\infty}(M), \tau_{0} \leq \tau<t_{1}$, and $q>3$ :

$$
\begin{aligned}
\|f\|_{L^{\frac{3}{2}\left(\Sigma_{\tau}\right)}} & \lesssim\|\nabla f\|_{L^{1}\left(\Sigma_{\tau}\right)}+\|f\|_{L^{1}\left(\Sigma_{\tau}\right)}, \\
\|f\|_{L^{\infty}\left(\Sigma_{\tau}\right)} & \lesssim\|\nabla f\|_{L^{q}\left(\Sigma_{\tau}\right)}+\|f\|_{L^{q}\left(\Sigma_{\tau}\right)} .
\end{aligned}
$$

In (68), the constant of the inequality depends also on $q$.

Proof. See [21, Lemma 2.1]. 16

Remark. The significance of Lemma 16 is that the constants of (67) and (68), i.e., the "Sobolev constants", are uniformly bounded over all the $\Sigma_{\tau}$ 's, $\tau_{0} \leq \tau<t_{1}$. In particular, they are controlled by the fundamental constants.

\footnotetext{
${ }^{16}$ Also essential to the proof of 68 is the "minimal radius" property of Proposition 14
} 
In addition, we can apply Lemma 16 along with the preceding remark in order to derive tensorial versions of Sobolev inequalities.

Proposition 17. Let $\tau_{0} \leq \tau<t_{1}$.

- For any $2 \leq p \leq 6, q>3$, and $\Psi \in \Gamma \underline{\mathcal{T}} \overline{\mathcal{T}} M$,

$$
\begin{aligned}
\|\Psi\|_{L^{p}\left(\Sigma_{\tau}\right)} & \lesssim\|\bar{\nabla} \Psi\|_{L^{2}\left(\Sigma_{\tau}\right)}+\|\Psi\|_{L^{2}\left(\Sigma_{\tau}\right)}, \\
\|\Psi\|_{L^{\infty}\left(\Sigma_{\tau}\right)} & \lesssim\|\bar{\nabla} \Psi\|_{L^{q}\left(\Sigma_{\tau}\right)}+\|\Psi\|_{L^{q}\left(\Sigma_{\tau}\right)} .
\end{aligned}
$$

The constants of the inequalities depend also on $p$ and $q$, respectively.

- In addition, for any $\Psi \in \Gamma \mathcal{\mathcal { T }} \overline{\mathcal{T}} M$ and $\Phi \in \Gamma \mathcal{T} M$,

$$
\begin{aligned}
\|\Psi\|_{L^{\infty}\left(\Sigma_{\tau}\right)} & \lesssim\left\|\bar{\nabla}^{2} \Psi\right\|_{L^{2}\left(\Sigma_{\tau}\right)}+\|\Psi\|_{L^{2}\left(\Sigma_{\tau}\right)}, \\
\|\Phi\|_{L^{\infty}\left(\Sigma_{\tau}\right)} & \lesssim\left\|D^{2} \Phi\right\|_{L^{2}\left(\Sigma_{\tau}\right)}+\|D \Phi\|_{L^{2}\left(\Sigma_{\tau}\right)}+\|\Phi\|_{L^{2}\left(\Sigma_{\tau}\right)} .
\end{aligned}
$$

Proof. The inequalities (69) and (70) are direct adaptations of the proof of the first part of [21, Prop. 4.7]; see also [21, Prop. 2.3], 21, Prop. 2.4], and [17, Cor. 2.7]. The main idea is to apply Lemma 16 to the scalar quantities $|\Psi|^{q}, q>1$. 17

Next, (71) is proved by applying (170) and (69) in succession. We can also obtain (72) by applying (70) and (69) in the following manner:

$$
\begin{aligned}
\|\Psi\|_{L^{\infty}\left(\Sigma_{\tau}\right)} & \lesssim\|D \Psi\|_{L^{4}\left(\Sigma_{\tau}\right)}+\|\Psi\|_{L^{4}\left(\Sigma_{\tau}\right)} \\
& \lesssim\left\|D^{2} \Psi\right\|_{L^{2}\left(\Sigma_{\tau}\right)}+\|D \Psi\|_{L^{2}\left(\Sigma_{\tau}\right)}+\|\Psi\|_{L^{2}\left(\Sigma_{\tau}\right)} .
\end{aligned}
$$

Note that for $\Phi \in \Gamma \mathcal{T} M=\Gamma \overline{\mathcal{T}} M$, we clearly have $|\bar{\nabla} \Phi| \lesssim|D \Phi|$.

5.3. Generalized Energy-Momentum Tensors. We now describe some general methods for obtaining global and local energy bounds for various spacetime quantities. Our focus will be on two general classes of "energy-momentum" tensor fields, abbreviated as EMTs, based on covariant wave-type and Maxwell-type equations.

The "wave equation" EMT was previously applied in 8, 17, in order to obtain higher-order estimates. In particular, in [17, this was used to bound the $L^{2}$-norms of covariant derivatives of the curvature in a vacuum spacetime. We will apply these tensor fields with analogous intentions. The "Maxwell" EMT is an adaptation of this process to solutions of "Maxwell-type" equations. We will use this tensor field to derive a priori energy estimates for the curvature $R$, thereby avoiding the more precise but more computationally complex Bel-Robinson tensor field.

Throughout this section, we will assume the following fields:

- Let $U, V \in \Gamma T^{r} M$ satisfy the covariant tensor wave equation

$$
\square_{g} U=V \text {, }
$$

where $\square_{g}$ is the tensorial wave operator $\square_{g}=g^{\alpha \beta} D_{\alpha \beta}$.

- Let $A \in \Gamma T^{r+2} M, B \in \Gamma T^{r+1} M$, and $C \in \Gamma T^{r+3} M$, with $A$ antisymmetric in its first two components and satisfing the Maxwell-type equations

$$
D^{\alpha} A_{\alpha \beta I}=B_{\beta I}, \quad D_{\gamma} A_{\alpha \beta I}+D_{\alpha} A_{\beta \gamma I}+D_{\beta} A_{\gamma \alpha I}=C_{\gamma \alpha \beta I} .
$$

We begin by describing the "wave equation" case as in [17. Define the EMT $Q_{w}[U] \in \Gamma S^{2} M$ and its associated current $P_{w}[U] \in \mathfrak{X}^{*}(M)$ by

$$
Q_{w}[U]_{\alpha \beta}=h\left(D_{\alpha} U, D_{\beta} U\right)-\frac{1}{2} g_{\alpha \beta} g^{\mu \nu} \cdot h\left(D_{\mu} U, D_{\nu} U\right)
$$

\footnotetext{
${ }^{17}$ We norm mixed tensor fields with respect to $h$ and $\gamma$.
} 


$$
P_{w}[U](X)=Q_{w}[U](X, T),
$$

where $h(\cdot, \cdot)$ denotes a full $h$-metric contraction of the unindexed components. Note the resemblance to the energy-momentum tensor (21) for the scalar field. In addition, we define the corresponding divergence quantities $D \cdot Q_{w}[U] \in \mathfrak{X}^{*}(M)$ and $D \cdot P_{w}[U] \in C^{\infty}(M)$ in the expected manner:

$$
D \cdot Q_{w}[U]_{\alpha}=D^{\beta} Q_{w}[U]_{\alpha \beta}, \quad D \cdot P_{w}[U]=D^{\alpha} P_{w}[U]_{\alpha} .
$$

The main properties of this wave EMT are listed below.

Proposition 18. The EMT $Q_{w}[U]$ satisfies the following properties:

- The following estimates hold:

$$
\begin{aligned}
\left|Q_{w}[U]\right| & \lesssim|D U|^{2}, \\
\left|D \cdot Q_{w}[U]\right| & \lesssim|D U|(|V|+|R[U]|)+|\pi||D U|^{2}, \\
\left|D \cdot P_{w}[U]\right| & \lesssim|D U|(|V|+|R[U]|)+|\pi||D U|^{2} .
\end{aligned}
$$

- For future causal $X, Y \in \mathfrak{X}(M)$,

$$
Q_{w}[U](X, Y) \geq 0,
$$

In particular,

$$
Q_{w}[U](T, T)=\frac{1}{2}|D U|^{2} .
$$

Proof. These follow immediately from a series of direct computations. For further details, consult [21, Prop. 4.13] and [17.

The "Maxwell-type" EMT is constructed analogously. Indeed, we define the EMT $Q_{m}[A] \in \Gamma S^{2} M$ and its associated current $P_{m}[A] \in \mathfrak{X}^{*}(M)$ by

$$
\begin{aligned}
Q_{m}[A]_{\alpha \beta} & =h\left(A_{\alpha \mu}, A_{\beta}{ }^{\mu}\right)-\frac{1}{4} g_{\alpha \beta} h\left(A_{\mu \nu}, A^{\mu \nu}\right), \\
P_{m}[A](X) & =Q_{m}[A](X, T),
\end{aligned}
$$

and define $D \cdot Q_{m}[A] \in \mathfrak{X}^{*}(M), D \cdot P_{m}[A] \in C^{\infty}(M)$ by

$$
D \cdot Q_{m}[A]_{\alpha}=D^{\beta} Q_{m}[A]_{\alpha \beta}, \quad D \cdot P_{m}[A]=D^{\alpha} P_{m}[A]_{\alpha} .
$$

Proposition 19. The EMT $Q_{m}[A]$ satisfies the following properties:

- The following estimates hold:

$$
\begin{aligned}
\left|Q_{m}[A]\right| & \lesssim|A|^{2}, \\
\left|D \cdot Q_{m}[A]\right| & \lesssim|A|(|B|+|C|)+|\pi||A|^{2}, \\
\left|D \cdot P_{m}[A]\right| & \lesssim|A|(|B|+|C|)+|\pi||A|^{2} .
\end{aligned}
$$

- For future causal $X, Y \in \mathfrak{X}(M)$,

$$
Q_{m}[A](X, Y) \geq 0 .
$$

In particular,

$$
Q_{m}[A](T, T)=\frac{1}{4}|A|^{2} .
$$

Proof. The proofs are analogous to those of Proposition[18, see [21, Prop. 4.14]. 
5.4. Global Energy Estimates. Next, we apply these generalized EMTs in order to establish both global and local energy estimates. By "global energy estimates", we mean $L^{2}$-estimates on entire timeslices, while by "local energy estimates", we refer to $L^{2}$-estimates on past null cones, i.e., "flux estimates".

We begin with general global estimates. Recall that Killing vector fields can be associated via Noether's theorem with conservation laws for quantities derived from (standard) energy-momentum tensors. Since we have no Killing fields in our setting, we have no hope for achieving such energy conservation. We can, however, think of $T$ as an "almost Killing" vector field, since its deformation tensor $\pi$ is uniformly bounded. In fact, by slightly modifying the process behind Noether's theorem, we can derive energy inequalities by taking advantage of this "almost Killing" condition for $T$ along with the generalized EMTs $Q_{w}[U]$ and $Q_{m}[A]$.

Lemma 20. Assume $U, V, A, B, C$ as before. If $\tau_{0} \leq \tau_{1}<\tau_{2}<t_{1}$, then

$$
\begin{aligned}
\|D U\|_{L^{2}\left(\Sigma_{\tau_{2}}\right)}^{2} & \lesssim\|D U\|_{L^{2}\left(\Sigma_{\tau_{1}}\right)}^{2}+\int_{\tau_{1}}^{\tau_{2}} \int_{\Sigma_{\tau}}\left(|D U|^{2}+|V|^{2}+|R[U]|^{2}\right) d \tau, \\
\|A\|_{L^{2}\left(\Sigma_{\tau_{2}}\right)}^{2} & \lesssim\|A\|_{L^{2}\left(\Sigma_{\tau_{1}}\right)}^{2}+\int_{\tau_{1}}^{\tau_{2}} \int_{\Sigma_{\tau}}\left(|A|^{2}+|B|^{2}+|C|^{2}\right) d \tau .
\end{aligned}
$$

Proof. First, for (83), we integrate $D \cdot P_{w}[U]$ over the slab $\Sigma_{\left[\tau_{1}, \tau_{2}\right]}$. 18 Applying the divergence theorem along with Proposition 5, we obtain the identity

$$
\int_{\Sigma_{\tau_{1}}} Q_{w}[U](T, T)-\int_{\Sigma_{\tau_{2}}} Q_{w}[U](T, T)=\int_{\tau_{1}}^{\tau_{2}}\left[\int_{\Sigma_{\tau}} n \cdot\left(D \cdot P_{w}[U]\right)\right] d \tau .
$$

By (63), (76), (78), then

$$
\begin{aligned}
\|D U\|_{L^{2}\left(\Sigma_{\tau_{2}}\right)}^{2} & \lesssim\|D U\|_{L^{2}\left(\Sigma_{\tau_{1}}\right)}^{2}+\int_{\tau_{1}}^{\tau_{2}} \int_{\Sigma_{\tau}}\left[|D U|(|V|+|R[U]|)+|D U|^{2}\right] d \tau \\
& \lesssim\|D U\|_{L^{2}\left(\Sigma_{\tau_{1}}\right)}^{2}+\int_{\tau_{1}}^{\tau_{2}} \int_{\Sigma_{\tau}}\left(|D U|^{2}+|V|^{2}+|R[U]|^{2}\right) d \tau .
\end{aligned}
$$

For (84), we repeat the above steps with $D \cdot P_{m}[A]$ in the place of $D \cdot P_{w}[U]$.

To obtain the specific a priori estimates we desire, we will apply Lemma 20 to the spacetime curvature $R$ and matter field: $D^{2} \phi$ in the E-S case, or $D F$ in the E-M case. For this, we will need to take advantage of the following facts:

- The curvature $R$ satisfies Maxwell-type equations. This includes

$$
D_{[\mu} R_{\alpha \beta] \gamma \delta} \equiv 0,
$$

i.e., the Bianchi idenitities, along with the divergence relations (25) and (26), which hold in the E-S and E-M settings, respectively.

- In the E-S case, $D \phi$ satisfies the following wave equation:

$$
\square_{g} D_{\alpha} \phi=R \cdot D_{\alpha} \phi
$$

This can be derived by differentiating (20) and commuting derivatives.

- In the E-M case, $F$ satisfies the following wave equation:

$$
\square_{g} F_{\alpha \beta}=2 R_{\alpha}{ }^{\lambda \mu}{ }_{\beta} F_{\lambda \mu}+R_{\alpha}^{\lambda} F_{\beta \lambda}-R_{\beta}^{\lambda} F_{\alpha \lambda} .
$$

This is derived by differentiating the Bianchi identity for $F$ in (22).

\footnotetext{
${ }^{18}$ Recall that $\Sigma_{\left[\tau_{1}, \tau_{2}\right]}=\left\{p \in M \mid \tau_{1} \leq t(p) \leq \tau_{2}\right\}$
} 
For every $\tau_{0} \leq \tau<t_{1}$, we define the global spacetime energy quantities

$$
\mathcal{E}^{1}(\tau)=\|R\|_{L^{2}\left(\Sigma_{\tau}\right)}+\left\|D^{2} \phi\right\|_{L^{2}\left(\Sigma_{\tau}\right)}, \quad \mathcal{E}^{1}(\tau)=\|R\|_{L^{2}\left(\Sigma_{\tau}\right)}+\|D F\|_{L^{2}\left(\Sigma_{\tau}\right)}
$$

in the E-S and E-M settings, respectively. 19

Proposition 21. In both the E-S and E-M cases, we have the energy inequality

$$
\mathcal{E}^{1}(\tau) \lesssim 1, \quad \tau_{0} \leq \tau<t_{1} .
$$

Proof. We begin with the E-S case. To handle the curvature, we apply (84), with $A=R$, and with $B$ and $C$ given by (25) and (85), in order to obtain

$$
\|R\|_{L^{2}\left(\Sigma_{\tau}\right)}^{2} \lesssim\|R\|_{L^{2}\left(\Sigma_{t_{0}}\right)}^{2}+\int_{t_{0}}^{\tau} \int_{\Sigma_{\tau^{\prime}}}\left(|R|^{2}+|D \phi|^{2}\left|D^{2} \phi\right|^{2}\right) d \tau^{\prime} .
$$

By (83), with $U=D \phi$, along with (866), we obtain the bound

$$
\left\|D^{2} \phi\right\|_{L^{2}\left(\Sigma_{\tau}\right)}^{2} \lesssim\left\|D^{2} \phi\right\|_{L^{2}\left(\Sigma_{t_{0}}\right)}^{2}+\int_{t_{0}}^{\tau} \int_{\Sigma_{\tau^{\prime}}}\left(\left|D^{2} \phi\right|^{2}+|D \phi|^{2}|R|^{2}\right) d \tau^{\prime} .
$$

Summing the above equations, recalling the uniform bound (40) for $D \phi$, and applying Grönwall's inequality to the result yields the desired bound.

The E-M case is derived in a completely analogous manner. We apply (83) and (84) as before, along with (26), (85), (87), and we obtain the bounds

$$
\begin{array}{r}
\|R\|_{L^{2}\left(\Sigma_{\tau}\right)}^{2} \lesssim\|R\|_{L^{2}\left(\Sigma_{t_{0}}\right)}^{2}+\int_{t_{0}}^{\tau} \int_{\Sigma_{\tau^{\prime}}}\left(|R|^{2}+|F|^{2}|D F|^{2}\right) d \tau^{\prime}, \\
\|D F\|_{L^{2}\left(\Sigma_{\tau}\right)}^{2} \lesssim\|D F\|_{L^{2}\left(\Sigma_{t_{0}}\right)}^{2}+\int_{t_{0}}^{\tau} \int_{\Sigma_{\tau^{\prime}}}\left(|D F|^{2}+|F|^{2}|R|^{2}\right) d \tau^{\prime} .
\end{array}
$$

Summing and applying Grönwall's inequality yields the desired inequality.

5.5. Local Energy Estimates. Local energy estimates are derived in a manner similar to the global estimates of Lemma 20. The main difference is that we integrate over the interior of a past null cone rather than over a time slab.

Fix $p \in M_{+}$; we normalize and foliate $\mathcal{N}^{-}(p)$ as before using $\left.T\right|_{p}$ and $t_{p}$. Fix also a time value $\tau_{0} \leq \tau<t(p)$ such that $t(p)-\tau<\min (\mathfrak{i}(p), 1)$. 20 We define

$$
\mathcal{N}_{\tau}=\mathcal{N}^{-}(p ; t(p)-\tau), \quad \mathcal{I}_{\tau}=\left\{q \in I^{-}(p) \mid t(q)>\tau\right\}, \quad \Sigma_{\tau}^{p}=I^{-}(p) \cap \Sigma_{\tau},
$$

where $I^{-}(p)$ is the chronological past of $p$, i.e., the set of points $q \in M$ reachable from $p$ via past timelike paths. Note that $\mathcal{I}_{\tau}$ is the region bounded by $\mathcal{N}_{\tau}$ and $\Sigma_{\tau}$, while $\Sigma_{\tau}^{p}$ is the part of $\Sigma_{\tau}$ in the interior of $\mathcal{N}^{-}(p)$.

The following lemma provides the main technical calculation for deriving local energy inequalities and justifies our previous definition of integration on $\mathcal{N}^{-}(p)$.

Lemma 22. If $\omega \in \mathfrak{X}^{*}(M)$, then

$$
\int_{\mathcal{I}_{\tau}} D^{\alpha} \omega_{\alpha}=\int_{\Sigma_{\tau}^{p}} \omega(T)+\int_{\mathcal{N}_{\tau}} \omega(L)
$$

Proof. See [21, Lemma 4.1] or [16].

\footnotetext{
${ }^{19}$ In later sections, we will also define and estimate the higher-order analogues $\mathcal{E}^{2}(\tau)$ and $\mathcal{E}^{3}(\tau)$. We adopt the abbreviation $\mathcal{E}^{1}(\tau)$ here in order to maintain consistency of notation.

${ }^{20}$ The stipulation $t(p)-\tau<1$ is purely a matter of convenience, in order to eliminate the dependence of various inequalities on the timespan $t(p)-\tau$.
} 
We are now prepared to derive the general local estimate.

Lemma 23. Assume $U, V, A, B, C$ as before. Then,

$$
\begin{aligned}
-\int_{\mathcal{N}_{\tau}} Q_{w}[U](T, L) \lesssim\|D U\|_{L^{2}\left(\Sigma_{\tau}^{p}\right)}^{2} & \\
& +\int_{\tau}^{t(p)} \int_{\Sigma_{\sigma}^{p}}\left(|D U|^{2}+|V|^{2}+|R[U]|^{2}\right) d \sigma \\
-\int_{\mathcal{N}_{\tau}} Q_{m}[A](T, L) \lesssim\|A\|_{L^{2}\left(\Sigma_{\tau}^{p}\right)}^{2} & \\
& +\int_{\tau}^{t(p)} \int_{\Sigma_{\sigma}^{p}}\left(|A|^{2}+|B|^{2}+|C|^{2}\right) d \sigma .
\end{aligned}
$$

Proof. Integrate $D \cdot P_{w}[U]$ over $\mathcal{I}_{\tau}$ and apply Lemma 22 and Proposition 5 to obtain

$$
\int_{\Sigma_{\tau}^{p}} Q_{w}[U](T, T)+\int_{\mathcal{N}_{\tau}} Q_{w}[U](T, L)=\int_{\tau}^{t(p)}\left(\int_{\Sigma_{\sigma}^{p}} n \cdot D \cdot P_{w}[U]\right) d \sigma .
$$

By (63), (76)), and (78), we obtain (88). Repeating the above steps, but with $P_{m}[A]$ in place of $P_{w}[U]$ and (80), (82) in place of (76), (78), we obtain (89).

Remark. Note that both $-Q_{w}[U](T, L)$ and $-Q_{m}[A](T, L)$ are nonnegative.

Next, we define the lower-order flux densities

$$
\begin{aligned}
& \rho^{1}(p, \tau)=-Q_{m}[R](T, L)-Q_{w}[D \phi](T, L), \\
& \rho^{1}(p, \tau)=-Q_{m}[R](T, L)-Q_{w}[F](T, L)
\end{aligned}
$$

on $\mathcal{N}_{\tau}$ in the E-S and E-M cases, respectively, and we define the corresponding flux

$$
\mathcal{F}^{1}(p, \tau)=\int_{\mathcal{N}_{\tau}} \rho^{1}(p, \tau) .
$$

We can now apply Lemma 23 directly to obtain the desired local energy estimates.

Proposition 24. In both the E-S and E-M cases, we have the local energy inequality

$$
\mathcal{F}^{1}(p, \tau) \lesssim 1
$$

Proof. For the E-S case, we first apply (89) with $A=R$ to obtain

$$
-\int_{\mathcal{N}_{\tau}} Q_{m}[R](T, L) \lesssim\|R\|_{L^{2}\left(\Sigma_{\tau}\right)}+\int_{\tau}^{t(p)}\left(\|R\|_{L^{2}\left(\Sigma_{\sigma}\right)}+\left\|D^{2} \phi\right\|_{L^{2}\left(\Sigma_{\sigma}\right)}\right) d \sigma \lesssim 1,
$$

where we also used Proposition 21. Similarly, applying (88) with $U=D \phi$ yields

$$
-\int_{\mathcal{N}_{\tau}} Q_{w}[D \phi](T, L) \lesssim 1
$$

The E-M case is handled analogously.

Remark. Estimates analogous to Proposition 24 hold for similar flux quantities associated with future regular null cones on the time interval $\left[\tau_{0}, t_{1}\right)$.

As of now, we have uniform control on the integrals over $\mathcal{N}_{\tau}$ of some quadratic quantities in $R$ and either $D^{2} \phi$ or $D F$, given explicitly by $-Q_{m}[R](T, L)$, $-Q_{w}[D \phi](T, L)$, and $-Q_{w}[F](T, L)$. The final step here is to determine exactly which components of $R$ and the matter field are controlled by Proposition 24 . 
Proposition 25. On $\mathcal{N}_{\tau}$, we have the comparisons

$$
\begin{aligned}
& -Q_{w}[U](T, L) \simeq\left|\bar{\nabla}_{4} U\right|^{2}+|\bar{\nabla} U|^{2}, \\
& -Q_{m}[A](T, L) \simeq\left|A_{43}\right|^{2}+\sum_{a=1}^{2}\left|A_{4 a}\right|^{2}+\sum_{a, b=1}^{2}\left|A_{a b}\right|^{2},
\end{aligned}
$$

where we have indexed with respect to adapted null frames on $\mathcal{N}^{-}(p)$, and where $A_{\alpha \beta} \in \Gamma T^{r} M$ is defined $\left(A_{\alpha \beta}\right)_{I}=A_{\alpha \beta I}$.

In other words, $-Q_{w}[U](T, L)$ controls all the components of $D U$ except for those of the form $D_{3} U_{I}$, i.e., derivatives transverse to $\mathcal{N}_{\tau}$. Similarly, $-Q_{m}[A](T, L)$ controls all the components of $A$ except those of the form $A_{3 a I}$. This coincides with standard results for scalar and Maxwell fields in Minkowski space.

Proof. We begin by defining the quantity $\varphi=g(T, L)$, which satisfies

$$
\left.\lim _{v \searrow 0} \varphi\right|_{(v, \omega)}=1, \quad\left|\mathcal{L}_{t_{p}} \varphi\right|=n \varphi^{-1} \cdot|L \varphi| \lesssim n \varphi^{-1}|\pi||L|^{2} \lesssim \varphi,
$$

where we have applied (53). Integrating the above along the null generators of $\mathcal{N}^{-}(p)$ and applying Grönwall's inequality yields the comparison $\varphi \simeq 1$. 21

Now, the proof is simply a matter of expanding $-Q_{w}[U](T, L)$ and $-Q_{m}[A](T, L)$ in terms of adapted null frames. From these computations, we obtain

$$
\begin{aligned}
& -Q_{w}[U](T, L)=\frac{1}{2} \varphi^{-1}\left|\bar{\not}_{4} U\right|^{2}+\frac{1}{2} \varphi|\bar{\not} U|^{2}, \\
& -Q_{m}[A](T, L)=\frac{1}{8} \varphi\left|A_{43}\right|^{2}+\frac{1}{2} \varphi^{-1} \sum_{a=1}^{2}\left|A_{4 a}\right|^{2}+\frac{1}{4} \varphi \sum_{a, b=1}^{2}\left|A_{a b}\right|^{2} .
\end{aligned}
$$

For some more details on these computations, see [21, Prop. 4.17]. The desired results now follow from the comparison $\varphi \simeq 1$.

We also note the following simple consequence of the proof of Proposition 25, which will be essential in later estimates involving past null cones.

Corollary 26. On $\mathcal{N}_{\tau}$, the null lapse $\vartheta$ satisfies $\vartheta \simeq 1$.

Proof. Since $\vartheta=n \varphi^{-1}$, where $\varphi$ is defined as in the proof of Proposition 25, then the desired estimate follows from (63) and the estimate $\varphi \simeq 1$.

Remark. As a result, the quantity $\vartheta$ will be negligible in estimates. In particular, the quantity $\bar{\nabla}_{4} U$ in Proposition 25 can be replaced by $\bar{\nabla}_{t_{p}} U$.

Lastly, we use Proposition 25 to see which null frame components of $R$ and the matter field are controlled using Proposition 24, First, for $R$, Proposition 25 implies that the components which are not controlled are those of the form $R_{3 a \alpha \beta}$. However, using the symmetry $R_{3 a \alpha \beta}=R_{\alpha \beta 3 a}$, we see in fact that the uncontrolled components are those of the form $R_{3 a 3 b}$. More explicitly, if $\psi$ denotes a null frame component of $R$ not of the form $R_{3 a 3 b}$, then

$$
\|\psi\|_{L^{2}\left(\mathcal{N}_{\tau}\right)} \lesssim 1
$$

Remark. The same result could also be obtained for $R$ by using instead the BelRobinson tensor field. This was done in [16, 17] in the E-V setting.

\footnotetext{
${ }^{21}$ Here, we also take advantage of the assumption $t(p)-\tau<1$.
} 
Next, in the E-S case, Proposition 25 implies that only the components $D_{3 \alpha} \phi$ of $D^{2} \phi$ are not controlled. Since $D^{2} \phi$ is symmetric, then in fact, only $D_{33} \phi$ cannot be controlled; for any other null frame component $\psi$ of $D^{2} \phi$, then

$$
\|\psi\|_{L^{2}\left(\mathcal{N}_{\tau}\right)} \lesssim 1
$$

Finally, in the E-M case, Proposition 25 implies that only the components $D_{3} F_{\alpha \beta}$ are not controlled. By rearranging components via the Maxwell equations, we can easily establish that the only truly uncontrollable components of $D F$ are those of the form $D_{3} F_{3 a}$; for any other null frame component $\psi$, then

$$
\|\psi\|_{L^{2}\left(\mathcal{N}_{\tau}\right)} \lesssim 1
$$

Remark. The second fundamental form $k$ also satisfies a tensorial covariant wave equation. One can also use this equation to derive analogous global and local energy estimates for $k$. Such global energy estimates are equivalent to those obtained from the elliptic estimates of (96), while the local estimates are a new result.

5.6. Preliminaries for Elliptic Estimates. The last class of a priori estimates we will establish are elliptic estimates on the $\Sigma_{\tau}$ 's. Before beginning this task, we first focus on some general concepts which will be used in these derivations.

A primary idea behind these elliptic estimates is the relations that exist between corresponding horizontal and spacetime objects, e.g., the curvatures $\mathcal{R}$ of the $\Sigma_{\tau}$ 's and $R$ of $M$. Our next step is to briefly describe such relations in an informal schematic manner. A more detailed account can be found throughout [21].

For any $A \in \Gamma \mathcal{T} M$, we let $\Pi A \in \Gamma \mathcal{T} M$ denote its projection to the $\Sigma_{\tau}$ 's. In particular, if $A$ is a vector field, then $\Pi A$ is its orthogonal projection onto the tangent bundles of the $\Sigma_{\tau}$ 's. If $A$ is fully covariant, then $\Pi A$ is given trivially by

$$
\Pi A\left(X_{1}, \ldots, X_{n}\right)=A\left(X_{1}, \ldots, X_{n}\right), \quad X_{1}, \ldots, X_{n} \in \underline{\mathfrak{x}}(M) .
$$

Furthermore, if $A$ is scalar, then $\Pi A=A$.

For simplicity, we will adopt an informal schematic language to describe tensorial expressions in an inexact form. We describe the conventions here merely by example; for a more exact description of the schematic notation, see 21. Consider the Gauss equations (32), which yields the informal schematic relation

$$
\mathcal{R} \approx \Pi R+k \cdot k \text {. }
$$

Here, the symbol represents a tensor product followed by a finite number of contractions and metric contractions. Moreover, the multiple terms of the form $k \cdot k$ in (32) are compacted into a single term in the informal equation (90).

We take advantage of such schematic relations by using the following lemma:

Lemma 27. If $\Phi \in \Gamma \mathcal{T} M$ is fully covariant, then we have the schematic relation

$$
\nabla \Pi \Phi \approx \Pi D \Phi+k \cdot \Pi(\Phi \cdot T) .
$$

Proof. Suppose $\Phi \in \Gamma T_{r}^{0} M, X \in \underline{\mathfrak{X}}(M)$, and $Z \in \Gamma \underline{T}^{r} M$. Then, by definition,

$$
\nabla_{X}(\Pi \Phi)(Z)=X(\Phi(Z))-\Phi\left(\nabla_{X} Z\right)=D_{X} \Phi(Z)+\Phi\left(D_{X} Z-\nabla_{X} Z\right) .
$$

The first term on the right is simply $\Pi D \Phi$ applied to $X \otimes Z$. Moreover, the quantity $D_{X} Z-\nabla_{X} Z$ can be expressed as a sum of terms of the form $k \cdot X \cdot Z \cdot T$. 22 The desired equation (91) follows as a result of the above observations.

\footnotetext{
${ }^{22}$ For example, if $Z \in \underline{\mathfrak{x}}(M)$, then $D_{X} Z-\nabla_{X} Z=-g\left(D_{X} Z, T\right) T=-k(X, Z) T$.
} 
For instance, considering (90), if we wish to control $\nabla \mathcal{R}$, then applying Lemma 27 with $\Phi \approx R$, we need only control terms of types $D R, k \cdot R \cdot T$, and $\nabla k \cdot k .23$ As a result of the above, we obtain the derivative bound

$$
|\nabla \mathcal{R}| \lesssim|D R|+|R||k|+|\nabla k||k|
$$

With a little more care, we can iterate the above argument using (90) in order to estimate higher derivatives of $\mathcal{R}$. In fact, such estimates for $\nabla^{2} \mathcal{R}$ will be necessary later when we establish higher-order elliptic estimates.

In addition to Lemma 27, we will also make use of the following general elliptic and Böchner estimates. For any fully symmetric $\xi \in \Gamma \underline{T}^{s} M, s>0$, we define

$$
\begin{aligned}
\nabla \cdot \xi \in \Gamma \underline{T}^{s-1} M, & (\nabla \cdot \xi)_{I}=\nabla^{a} \xi_{a I}, \\
\nabla \times \xi \in \Gamma \underline{T}^{s+1} M, & (\nabla \times \xi)_{a b I}=\nabla_{a} \xi_{b I}-\nabla_{b} \xi_{a I}, \\
\operatorname{tr} \xi \in \Gamma \underline{T}^{s-2} M, & \begin{cases}(\operatorname{tr} \xi)_{I}=\gamma^{a b} \xi_{a b I} & s>1, \\
0 & s=1,\end{cases}
\end{aligned}
$$

i.e., the divergence, curl, and trace of $\xi$, respectively.

Lemma 28. If $\xi \in \Gamma \underline{\mathcal{T}} M$ is fully symmetric and nonscalar, then

$$
\int_{\Sigma_{\tau}}|\nabla \xi|^{2} \lesssim \int_{\Sigma_{\tau}}\left(|\nabla \cdot \xi|^{2}+\frac{1}{2}|\nabla \times \xi|^{2}+|\mathcal{R}||\xi|^{2}\right) .
$$

Moreover, for any $\Psi \in \Gamma \underline{\mathcal{T}} M$ and $\phi \in C^{\infty}(M)$,

$$
\begin{aligned}
\int_{\Sigma_{\tau}}\left|\nabla^{2} \Psi\right|^{2} & \lesssim \int_{\Sigma_{\tau}}\left(|\Delta \Psi|^{2}+|\mathcal{R}||\nabla \Psi|^{2}+\frac{1}{2}|\mathcal{R}|^{2}|\Psi|^{2}\right) \\
\int_{\Sigma_{\tau}}\left|\nabla^{2} \phi\right|^{2} & \lesssim \int_{\Sigma_{\tau}}\left(|\Delta \phi|^{2}+|\mathcal{R}||\nabla \phi|^{2}\right) .
\end{aligned}
$$

Proof. See [21, Lemma 2.3, Cor. 2.5]; see also [7, Ch. 2].

In particular, Lemma 28 will be useful for elliptic estimates on $k$ and $n$. Variants of Lemma 28 will also be essential for controlling the local null geometry.

5.7. Elliptic Estimates. Recall that our primary task in proving Theorem 6 is to control the horizontal energy quantities $\mathfrak{K}(\tau), \mathfrak{R}(\tau)$, and $\mathfrak{f}(\tau)$ defined in (44)-(46). To accomplish this, we must apply various elliptic estimates in conjunction with energy estimates derived using EMTs, for example, Propositions 21] and 24. In addition, we must control $L^{2}$-norms of derivatives of the lapse $n$, as well as obtain an $L^{\infty}$-bound for $\mathcal{L}_{t} n$. These will be necessary in order to control the geometry of null cones and derive higher-order elliptic estimates.

We begin here by deriving lower-order a priori elliptic estimates for $k$ and $\mathcal{R}$, as well as the horizontal formulation $\mathfrak{f}$ of the matter field. These estimates are the E-S and E-M analogues for the elliptic estimates of [17, Sec. 8] in the vacuum case. The main difference here is that we must also bound the matter field.

Remark. The proofs of [17, Sec. 8] were adapted for maximal foliations. Here, we simplify the process by taking advantage of the volume bounds of Proposition 12.

Recall that the "horizontal matter field quantities" $\mathfrak{f}$ are given as follows:

- In the E-S setting, this consists of the scalar field $\phi$ itself and $\mathcal{L}_{t} \phi$.

\footnotetext{
${ }^{23}$ Note that $|\Pi \Phi| \leq|\Phi|$ for any $\Phi \in \Gamma \mathcal{T} M$.
} 
- In the E-M setting, this consists of the pair $E, H \in \underline{\mathfrak{X}}(M)$ representing the electromagnetic decomposition of $F$.

Remark. Recall that given a set orientation of $M$ (or equivalently, of $\Sigma_{\tau_{0}}$ ), the electromagnetic decomposition of $F$ is given by the formulas

$$
E_{i}=F_{\alpha i} T^{\alpha}, \quad H_{i}={ }^{\star} F_{\alpha i} T^{\alpha}=\frac{1}{2} \epsilon_{\alpha i}{ }^{\beta \gamma} F_{\beta \gamma} T^{\alpha} .
$$

First, by Proposition 12 and (40), we have the following trivial bounds:

Lemma 29. For any $1 \leq p \leq \infty$ and $\tau_{0} \leq \tau<t_{1}$, we have the estimates

$$
\begin{aligned}
\|k\|_{L^{p}\left(\Sigma_{\tau}\right)}+\|\nabla \phi\|_{L^{p}\left(\Sigma_{\tau}\right)}+\left\|\mathcal{L}_{t} \phi\right\|_{L^{p}\left(\Sigma_{\tau}\right)} & \lesssim 1, \\
\|k\|_{L^{p}\left(\Sigma_{\tau}\right)}+\|E\|_{L^{p}\left(\Sigma_{\tau}\right)}+\|H\|_{L^{p}\left(\Sigma_{\tau}\right)} & \lesssim 1
\end{aligned}
$$

in the $E-S$ and $E-M$ cases, respectively.

The next batch of elliptic estimates are consequences of various schematic relations between horizontal and spacetime objects.

Proposition 30. For any $\tau_{0} \leq \tau<t_{1}$, we have the elliptic estimates

$$
\|\mathcal{R}\|_{L^{2}\left(\Sigma_{\tau}\right)}+\|\nabla k\|_{L^{2}\left(\Sigma_{\tau}\right)} \lesssim 1
$$

Moreover, for any $\tau_{0} \leq \tau<t_{1}$, the estimates

$$
\begin{aligned}
\left\|\nabla^{2} \phi\right\|_{L^{2}\left(\Sigma_{\tau}\right)}+\left\|\nabla\left(\mathcal{L}_{t} \phi\right)\right\|_{L^{2}\left(\Sigma_{\tau}\right)} & \lesssim 1, \\
\|\nabla E\|_{L^{2}\left(\Sigma_{\tau}\right)}+\|\nabla H\|_{L^{2}\left(\Sigma_{\tau}\right)} & \lesssim 1
\end{aligned}
$$

hold in the $E-S$ and $E-M$ cases, respectively.

Proof. From (32), we immediately obtain

$$
\|\mathcal{R}\|_{L^{2}\left(\Sigma_{\tau}\right)} \lesssim\|R\|_{L^{2}\left(\Sigma_{\tau}\right)}+\|k\|_{L^{\infty}(\Sigma)}\|k\|_{L^{2}\left(\Sigma_{\tau}\right)} \lesssim 1+\|R\|_{L^{2}\left(\Sigma_{\tau}\right)} .
$$

To bound $\nabla k$, we appeal to (93). From (33) and the CMC gauge condition, we obtain $|\nabla \cdot k|+|\nabla \times k| \lesssim|R|$, hence by (93), Lemma 29, and the above,

$$
\|\nabla k\|_{L^{2}\left(\Sigma_{\tau}\right)} \lesssim\|R\|_{L^{2}\left(\Sigma_{\tau}\right)}+\left(\int_{\Sigma_{\tau}}|\mathcal{R}||k|^{2}\right)^{\frac{1}{2}} \lesssim 1+\|R\|_{L^{2}\left(\Sigma_{\tau}\right)} .
$$

Combining the above and applying Proposition 21 yields (96).

For the matter field estimates of (97), we appeal to the informal schematic language. In the E-S setting, we have the schematic relations

$$
\nabla \phi \approx \Pi D \phi, \quad n^{-1} \mathcal{L}_{t} \phi \approx \Pi(D \phi \cdot T) .
$$

Similarly, in the E-M setting,

$$
E \approx \Pi(F \cdot T), \quad H \approx \Pi(V \cdot F \cdot T),
$$

where $V$ is the volume form for $M$ (given an orientation of $M$ ). The estimates (97) then follow immediately after applying Lemma 27 and Proposition 21 to the schematic equations (98) and (99). For details, see [21, Lemma 6.5].

We can use the lapse equation (61) in order to derive some higher-order a priori bounds for $n$. These are described in the next two propositions: 
Proposition 31. For any $\tau_{0} \leq \tau<t_{1}$, we have the estimates

$$
\begin{aligned}
\left\|\nabla^{2} n\right\|_{L^{2}\left(\Sigma_{\tau}\right)} & \lesssim 1, \\
\left\|-\Delta\left(\mathcal{L}_{t} n\right)+\left[|k|^{2}+\operatorname{Ric}(T, T)\right] \mathcal{L}_{t} n\right\|_{L^{2}\left(\Sigma_{\tau}\right)} & \lesssim 1, \\
\left\|\nabla\left(\mathcal{L}_{t} n\right)\right\|_{L^{2}\left(\Sigma_{\tau}\right)}+\left\|\mathcal{L}_{t} n\right\|_{L^{2}\left(\Sigma_{\tau}\right)} & \lesssim 1 .
\end{aligned}
$$

Proof. Equations (40), (61), and Proposition 12 imply $\|\Delta n\|_{L^{\infty}\left(\Sigma_{\tau}\right)} \lesssim 1$. Then,

$$
\int_{\Sigma_{\tau}}\left|\nabla^{2} n\right|^{2} \lesssim \int_{\Sigma_{\tau}}\left(|\Delta n|^{2}+|\mathcal{R}||\nabla n|^{2}\right) \lesssim 1+\int_{\Sigma_{\tau}}|\mathcal{R}|^{2}
$$

by (94) and Lemma 29, applying (96) results in (100).

The proofs of (101) and (102) are a bit more involved. Recall that $\mathcal{L}_{t}$ applied to a scalar $\phi$ is the same as $n T \phi$. We begin by commuting $\mathcal{L}_{t}$ with $\Delta$ to obtain

$$
\left|\Delta \mathcal{L}_{t} n-\mathcal{L}_{t} \Delta n\right| \lesssim|n||k|\left|\nabla^{2} n\right|+(|\nabla n \cdot k|+|n \cdot \nabla k|)|\nabla n|,
$$

where we also used (34). Applying (40), Proposition 30, and (100) yields

$$
\left\|\Delta \mathcal{L}_{t} n-\mathcal{L}_{t} \Delta n\right\|_{L^{2}\left(\Sigma_{\tau}\right)} \lesssim 1
$$

Next, differentiating (61), we have

$$
\mathcal{L}_{t} \Delta n=\left[|k|^{2}+\operatorname{Ric}(T, T)\right] \mathcal{L}_{t} n+n \cdot \mathcal{L}_{t}|k|^{2}+n \cdot \mathcal{L}_{t}[\operatorname{Ric}(T, T)] .
$$

Moreover, applying (35) and (63), we obtain

$$
\left.\left|n \cdot \mathcal{L}_{t}\right| k\right|^{2}|\lesssim 1+| R|+| \nabla^{2} n \mid .
$$

If we let $\mathfrak{F}$ denote $D \phi$ in the E-S case and $F$ in the E-M case, then by (19) and (40), we see that $\mid D$ Ric $|\lesssim| D \mathfrak{F} \mid$. As a result,

$$
\left|n \cdot \mathcal{L}_{t}[\operatorname{Ric}(T, T)]\right| \lesssim|D \operatorname{Ric}|+\left|D_{T} T\right||\operatorname{Ric}| \lesssim 1+|D \mathfrak{F}| .
$$

Combining Proposition 21, (100), (103), and the above, we obtain (101).

Define now the scalar

$$
P=-\Delta \mathcal{L}_{t} n+\left[|k|^{2}+\operatorname{Ric}(T, T)\right] \mathcal{L}_{t} n .
$$

Multiplying $P$ by $\mathcal{L}_{t} n$, integrating by parts, and decomposing $|k|^{2}$, then

$$
\begin{aligned}
\int_{\Sigma_{\tau}}\left(\left|\nabla \mathcal{L}_{t} n\right|^{2}+\frac{1}{3} t_{1}^{2}\left|\mathcal{L}_{t} n\right|^{2}\right) & \leq \int_{\Sigma_{\tau}}\left[\left|\nabla \mathcal{L}_{t} n\right|^{2}+\left(\frac{1}{3} \tau^{2}+|\hat{k}|^{2}+\operatorname{Ric}(T, T)\right)\left|\mathcal{L}_{t} n\right|^{2}\right] \\
& =\int_{\Sigma_{\tau}}|P|\left|\mathcal{L}_{t} n\right|,
\end{aligned}
$$

where we have also used the strong energy condition satisfied by both the E-S and E-M models. Applying a weighted Cauchy inequality to the right-hand side along with (101) yields (102), as desired.

Proposition 32. For any $\tau_{0} \leq \tau<t_{1}$,

$$
\left\|\nabla^{2} \mathcal{L}_{t} n\right\|_{L^{2}\left(\Sigma_{\tau}\right)}+\left\|\mathcal{L}_{t} n\right\|_{L^{\infty}\left(\Sigma_{\tau}\right)} \lesssim 1
$$


Proof. By (40), (94), and (96),

$$
\begin{aligned}
\left\|\nabla^{2} \mathcal{L}_{t} n\right\|_{L^{2}\left(\Sigma_{\tau}\right)} \lesssim\left\|\Delta \mathcal{L}_{t} n\right\|_{L^{2}\left(\Sigma_{\tau}\right)}+\|\mathcal{R}\|_{L^{2}\left(\Sigma_{\tau}\right)}^{\frac{1}{2}}\left\|\nabla \mathcal{L}_{t} n\right\|_{L^{4}\left(\Sigma_{\tau}\right)} \\
\lesssim\left\|\mathcal{L}_{t} n\right\|_{L^{2}\left(\Sigma_{\tau}\right)}+\left\|-\Delta \mathcal{L}_{t} n+\left[|k|^{2}+\operatorname{Ric}(T, T)\right] \mathcal{L}_{t} n\right\|_{L^{2}\left(\Sigma_{\tau}\right)} \\
\quad+\left\|\nabla \mathcal{L}_{t} n\right\|_{L^{4}\left(\Sigma_{\tau}\right)} .
\end{aligned}
$$

Applying Proposition 31 then

$$
\left\|\nabla^{2} \mathcal{L}_{t} n\right\|_{L^{2}\left(\Sigma_{\tau}\right)} \lesssim 1+\left\|\nabla \mathcal{L}_{t} n\right\|_{L^{4}\left(\Sigma_{\tau}\right)} .
$$

Next, by the Sobolev estimate [17, Cor 2.7], along with (102), then

$$
\begin{aligned}
\left\|\nabla^{2} \mathcal{L}_{t} n\right\|_{L^{2}\left(\Sigma_{\tau}\right)} & \lesssim 1+\left\|\nabla^{2} \mathcal{L}_{t} n\right\|_{L^{2}\left(\Sigma_{\tau}\right)}^{\frac{3}{4}}\left\|\nabla \mathcal{L}_{t} n\right\|_{L^{2}\left(\Sigma_{\tau}\right)}^{\frac{1}{4}}+\left\|\nabla \mathcal{L}_{t} n\right\|_{L^{2}(\Sigma)} \\
& \lesssim\left(1+\epsilon^{-4}\right)+\epsilon^{\frac{4}{3}}\left\|\nabla^{2} \mathcal{L}_{t} n\right\|_{L^{2}\left(\Sigma_{\tau}\right)}
\end{aligned}
$$

for any $\epsilon>0$. Taking $\epsilon$ sufficiently small, we obtain the desired bound for $\nabla^{2} \mathcal{L}_{t} n$. Lastly, we can bound $\mathcal{L}_{t} n$ in $L^{\infty}$ by the Sobolev bound (71) and the above.

The uniform bound for $\mathcal{L}_{t} n$ in Proposition 32, omitted in the earliest versions of the works in the E-V setting, is essential for controlling null injectivity radii.

\section{Local Regularity of Null Cones}

In this section, we will summarize what is the most technically demanding step in the proof of Theorem [6 control of the geometry of regular past null cones. This step will be crucial for applying the generalized representation formula for tensor wave equations, which will be needed in order to obtain the higher-order energy bounds required to complete the proof of Theorem 6 .

Throughout this section, we fix an arbitrary point $p \in M_{+}$, and we normalize and foliate the regular past null cone $\mathcal{N}^{-}(p)$ by $\left.T\right|_{p}$ and $t_{p}=t(p)-t$, as usual. In addition, we fix a constant $0<\delta_{0} \leq \min (\mathfrak{i}(p), 1)$, and we define the segment

$$
\mathcal{N}=\mathcal{N}^{-}\left(p ; \delta_{0}\right)=\left\{z \in \mathcal{N}^{-}(p) \mid t_{p}(z)<\delta_{0}\right\} .
$$

The main result of this section states roughly the following:

- The past null injectivity radius $\mathfrak{i}(p)$ of $p$ is bounded below by some constant $\delta>0$ depending only on the fundamental constants.

- For small enough $\delta_{0}$, depending only on the fundamental constants, the Ricci coefficients $\chi, \chi, \zeta, \eta$ of $\mathcal{N}^{-}(p)$ (see Section 4.4) can be controlled on $\mathcal{N}$ in various norms by the fundamental constants.

The explicit result is stated in Theorem 33, and a detailed proof of the theorem is available in [21, Ch. 7-8]. 24 Due to the prohibitive length and the level of technical detail of the argument, we omit the proof of this theorem in this paper and refer the reader to 21 for details. 25 On the other hand, we do provide a brief outline of the main components of the proof at the end of this section. Our focus, however, will be on the consequences of Theorem 33 in relation to the breakdown problem.

\footnotetext{
${ }^{24}$ See also [12, 14, 16, 19, 24, 25] for earlier results in Einstein-vacuum settings.

${ }^{25}$ In fact, a complete accounting of the details of the proof of Theorem 33 would more than double the length of this paper!
} 
6.1. Motivation and Past Results. In Riemannian geometry, one generally requires $L^{\infty}$-bounds on the curvature in order to derive uniform lower bounds for the injectivity radius. Similarly, for the Lorentzian case, given an $L^{\infty}$-bound for $R$, we can control the Ricci coefficients $\chi, \underline{\chi}, \zeta$, and $\underline{\eta}$ without too much effort. Uniform bounds for the null injectivity radius would also follow.

Unfortunately, we will only have the local flux bounds of Proposition 24 here, which makes our task tremendously more difficult. Indeed, bounding the Ricci coefficients using $L^{2}$ rather than $L^{\infty}$-estimates will necessitate the use of sharp trace estimates along the generators of null cones, which then requires the geometric Littlewood-Paley theory of [13] and the resulting Besov estimates. This process is responsible for much of the technical difficulties in the breakdown problem.

Such $L^{2}$-curvature results were first obtained by S. Klainerman and I. Rodnianski in [12, 14, 16. The results of [12, 14, however, applied only to truncated null cones and hence were not directly applicable to the breakdown problem. To address this issue, Q. Wang, in 24, 25], extended the estimates in [12, 14 to past null cones with vertex initial data by keeping track of scaling factors at every step. 26 Later, D. Parlongue, in [19, revisited this argument for time-foliated null cones.

In all the above, an essential assumption is that the spacetime is vacuum. The primary contribution of this section is the extension of this family of results to the E-S and E-M settings. We offer now a more detailed comparison between the current results and its predecessors:

- The papers 12, 14, 19, all considered the case of truncated regular null cones with prescribed spherical initial data. In contrast, we adopt the setting of [24, 25] and consider the case of regular null cones with initial data given by a vertex point. As mentioned before, this is the type of result needed by the breakdown problems in this text as well as in [17, 19].

- Unlike [12, 14, 24, 25, which bounded the Ricci coefficients with respect to the geodesic foliation, we obtain these bounds in terms of the time foliation, like in [19. This has the advantage of being able to interface directly with the breakdown problem. In this "gauge", we will need the regularity properties of the time foliation in order to bound the Ricci coefficients. However, this also results in improved estimates for both $\underline{\eta}$ and $\underline{\chi}$.

- While [12, 14, 16, 19, 24, 25, dealt exclusively with the Einstein-vacuum setting, here we extend the results to E-S and E-M spacetimes. In particular, the "curvature flux" defined in [12, 16, 19, 24, 25] is replaced by an analogous flux quantity involving both the curvature and the matter field; see Proposition 24. Furthermore, several of the structure equations governing the Ricci coefficients now contain additional terms reflecting the contributions from the nontrivial matter field.

- The works [12, 14, 19, 24, 25] all dealt only with the special case of very small curvature flux and initial values, over a unit interval along (possibly truncated) null cones. This is, however, not directly applicable to the breakdown problem, since in this setting we can only stipulate bounded, not small, curvature flux. In our case, Propositions 21 and 24 only established the existence of some possibly large a priori energy and flux bounds. On the other hand, we can in the breakdown problem work in only a very

\footnotetext{
${ }^{26}$ The works [24, 25] also addressed an error in [12], which assumed a Besov bound that does not hold. In fact, a significant amount of effort in [24, 25] is dedicated to addressing this issue.
} 
small interval along null cones. Heuristically, one can see that this "large flux, small interval" case relates to the "small flux, unit interval" case via a rescaling argument. However, we handle the "large flux, small interval" setting directly in the statement and proof of Theorem 33 .

- The only paper in the existing literature to address the null injectivity radius is [16, which was separated from the remaining components [12, 14] in the overall proof. In the time foliation case, however, the interplay between the "Ricci coefficients" and the "null injectivity radius" portions of the proof is more subtle and must be addressed in tandem.

6.2. Integral Norms. In order to state the main result, we must first define the relevant integral norms used within. We begin with natural integral norms on $\mathcal{N}$ (or $\mathcal{N}^{-}(p)$ ): for any $1 \leq q<\infty$ and $\Psi \in \Gamma \underline{\mathcal{T}} \overline{\mathcal{T}} \mathcal{N}^{-}(p)$, we define

$$
\|\Psi\|_{L^{q}(\mathcal{N})}=\left(\int_{\mathcal{N}}|\Psi|^{q}\right)^{\frac{1}{q}}, \quad\|\Psi\|_{L^{\infty}(\mathcal{N})}=\sup _{z \in \mathcal{N}} \mid \Psi \|_{z},
$$

where mixed tensors are normed with respect to $h$ and $\lambda$, and where the above integral over $\mathcal{N}$ is defined using the formula (50). In addition, we will make use of the following "null Sobolev" norm on $\mathcal{N}$ :

$$
\|\Psi\|_{\mathcal{H}^{1}(\mathcal{N})}=\left\|\overline{\mathbb{X}}_{t_{p}} \Psi\right\|_{L^{2}(\mathcal{N})}+\|\bar{\nabla} \Psi\|_{L^{2}(\mathcal{N})}+\left\|t_{p}^{-1} \Psi\right\|_{L^{2}(\mathcal{N})} .
$$

Given $1 \leq q<\infty$ and $1 \leq r \leq \infty$, we also define the following iterated norms:

$$
\begin{aligned}
\|\Psi\|_{L_{t}^{q} L^{r}(\mathcal{N})} & =\left(\int_{0}^{\delta_{0}}\|\Psi\|_{L^{r}\left(\mathcal{S}_{v}\right)}^{q} d v\right)^{\frac{1}{q}}, \\
\|\Psi\|_{L_{t}^{\infty} L^{r}(\mathcal{N})} & =\sup _{0<v<\delta_{0}}\|\Psi\|_{L^{r}\left(\mathcal{S}_{v}\right)} .
\end{aligned}
$$

As before, we use $\mathcal{S}_{v}$ to denote the level set $\left\{z \in \mathcal{N} \mid t_{p}(z)=v\right\}$, while $L^{r}\left(\mathcal{S}_{v}\right)$ refers to the tensorial $L^{r}$-norm on $\mathcal{S}_{v}$ with respect to the induced metric $\lambda$ on $\mathcal{S}_{v}$. Since $\vartheta \simeq 1$ on $\mathcal{N}$ due to Corollary 26, then by (50),

$$
\|\Psi\|_{L_{t}^{q} L^{q}(\mathcal{N})} \simeq\|\Psi\|_{L^{q}(\mathcal{N})}, \quad 1 \leq q \leq \infty
$$

i.e., the $L_{t}^{q} L^{q}$ and $L^{q}$-norms on $\mathcal{N}$ are equivalent.

Recall that $\mathcal{N}$ can be parametrized by the $t_{p}$-value $0<v<\delta_{0}$ and a spherical parameter $\omega \in \mathbb{S}^{2}$. We can then define additional iterated norms with respect to this parametrization: for any $1 \leq q, r<\infty$, we define

$$
\begin{aligned}
\|\Psi\|_{L_{t}^{q} L_{\omega}^{r}(\mathcal{N})} & =\left[\int_{0}^{\delta_{0}}\left(\left.\int_{\mathbb{S}^{2}}|\Psi|^{r}\right|_{(v, \omega)} d \omega\right)^{\frac{q}{r}} d v\right]^{\frac{1}{q}}, \\
\|\Psi\|_{L_{t}^{\infty} L_{\omega}^{r}(\mathcal{N})} & =\sup _{0<v<\delta_{0}}\left(\left.\int_{\mathbb{S}^{2}}|\Psi|^{r}\right|_{(v, \omega)} d \omega\right)^{\frac{1}{r}}, \\
\|\Psi\|_{L_{t}^{q} L_{\omega}^{\infty}(\mathcal{N})} & =\left[\int_{0}^{\delta_{0}}\left(\sup _{\omega \in \mathbb{S}^{2}} \mid \Psi \|_{(v, \omega)}\right)^{q} d v\right]^{\frac{1}{q}} .
\end{aligned}
$$


We can reverse the order of integration to obtain additional useful norms:

$$
\begin{aligned}
\|\Psi\|_{L_{\omega}^{r} L_{t}^{q}(\mathcal{N})} & =\left[\int_{\mathbb{S}^{2}}\left(\left.\int_{0}^{\delta_{0}}|\Psi|^{q}\right|_{(v, \omega)} d v\right)^{\frac{r}{q}} d \omega\right]^{\frac{1}{r}}, \\
\|\Psi\|_{L_{\omega}^{r} L_{t}^{\infty}(\mathcal{N})} & =\left[\int_{\mathbb{S}^{2}}\left(\sup _{0<v<\delta_{0}} \mid \Psi \|_{(v, \omega)}\right)^{r} d \omega\right]^{\frac{1}{r}}, \\
\|\Psi\|_{L_{\omega}^{\infty} L_{t}^{q}(\mathcal{N})} & =\sup _{\omega \in \mathbb{S}^{2}}\left(\int_{0}^{\delta_{0}} \mid \Psi \|_{(v, \omega)}^{q} d \omega\right)^{\frac{1}{q}} .
\end{aligned}
$$

Lastly, the $L_{t}^{\infty} L_{\omega}^{\infty}(\mathcal{N})$ and $L_{\omega}^{\infty} L_{t}^{\infty}(\mathcal{N})$-norms coincide with the $L^{\infty}(\mathcal{N})$-norm.

Later, we will see that the parameter $L_{t}^{q} L_{\omega}^{r}$-norms are comparable to rescalings of the natural iterated $L_{t}^{q} L^{r}$-norms. Of the reverse parametrized norms, the most important will be the "trace" $L_{\omega}^{\infty} L_{t}^{2}$-norms and $L_{\omega}^{q} L_{t}^{\infty}$-norms.

6.3. The Main Result. With all of the appropriate norms defined, we can now state the main result of this section.

Theorem 33. For any $q \in M_{+}$, the following hold:

- There exists a constant $\delta_{0}>0$, depending only on the fundamental constants, such that $\mathfrak{i}(q)>\min \left(\delta_{0}, t(q)-\tau_{0}\right)$.

- Letting $\mathcal{N}_{q}=\mathcal{N}^{-}\left(q ; \min \left(\delta_{0}, t(q)-\tau_{0}\right)\right)$, then the following estimates hold:

$$
\begin{array}{r}
\left\|\vartheta(\operatorname{tr} \chi)-\frac{2}{t_{q}}\right\|_{L_{\omega}^{\infty} L_{t}^{2}\left(\mathcal{N}_{q}\right)}+\|\hat{\chi}\|_{L_{\omega}^{\infty} L_{t}^{2}\left(\mathcal{N}_{q}\right)}+\|\zeta\|_{L_{\omega}^{\infty} L_{t}^{2}\left(\mathcal{N}_{q}\right)} \lesssim 1, \\
\left\|\vartheta(\operatorname{tr} \chi)-\frac{2}{t_{q}}\right\|_{\mathcal{H}^{1}\left(\mathcal{N}_{q}\right)}+\|\hat{\chi}\|_{\mathcal{H}^{1}\left(\mathcal{N}_{q}\right)}+\|\zeta\|_{\mathcal{H}^{1}\left(\mathcal{N}_{q}\right)} \lesssim 1,
\end{array}
$$

where $\chi$ and $\zeta$ refer to the corresponding Ricci coefficients of $\mathcal{N}^{-}(q)$.

- In addition, for $\mathcal{N}_{q}$ as above, we have

$$
\begin{aligned}
\left\|\vartheta(\operatorname{tr} \chi)-\frac{2}{t_{q}}\right\|_{L^{\infty}\left(\mathcal{N}_{q}\right)}+\left\|t_{q}^{\frac{3}{2}} \not(\operatorname{tr} \chi)\right\|_{L_{\omega}^{2} L_{t}^{\infty}\left(\mathcal{N}_{q}\right)} & \lesssim 1, \\
\left\|t_{q}^{\frac{3}{2}} \mu\right\|_{L_{\omega}^{2} L_{t}^{\infty}\left(\mathcal{N}_{q}\right)}+\|\mu\|_{L^{2}\left(\mathcal{N}_{q}\right)} & \lesssim 1,
\end{aligned}
$$

where $\mu$ is the mass aspect function on $\mathcal{N}_{q}$ defined in (57).

Remark. An analogue of Theorem 33 holds for future null cones $\mathcal{N}^{+}(p)$.

With greater care throughout the proof of Theorem 33. we could in fact be more precise about the bounds for the Ricci coefficients in (109) and (110), and how they are affected by the fundamental constants. Such a task is significantly simpler in the geodesically foliated E-V settings examined in [12, 24, 25], since in these cases, the only external parameter in the problems is the curvature flux.

Again, the details of the proof of Theorem 33 are left to [21, Ch. 7-8] due to its length and the amount of technical background involved. A brief summary of the proof is given at the end of this section, after first discussing the consequences of Theorem 33 pertaining to the current breakdown problem. 
6.4. Some Basic Consequences. We now list some basic consequences of Theorem 33 which will be useful in upcoming analyses involving null cones. Recall that the induced metrics on the $\mathcal{S}_{v}$ 's are denoted by $\lambda$. We define the "rescaled" metrics on these level sets by $\bar{\lambda}=\left(t_{p}\right)^{-2} \lambda$. This rescaling is essential, since in the case of null cones with vertex initial data, the uniformities occur with respect to $\bar{\lambda}$ rather than $\lambda$. For example, from Proposition 8 , we observe that $\bar{\lambda}$ tends toward the standard Euclidean metric on $\mathbb{S}^{2}$ (rescaled by a factor of $\left.n(p)^{2}\right)$ as $t_{p} \searrow 0$.

One consequence of Proposition 8 and Theorem 33, then, is that as long as the timespan $\delta_{0}$ of $\mathcal{N}$ is sufficiently small, then $\bar{\lambda}$ does not differ much from the standard Euclidean metric on $\mathbb{S}^{2}$. Another related result is that for similarly small $\delta_{0}$, then the volume forms $\bar{V}$ on the $\mathcal{S}_{v}$ 's with respect to $\bar{\lambda}$ do not differ much from that of the standard Euclidean metric on $\mathbb{S}^{2}$. Precise statements, in terms of transported coordinate systems, can be found in [21, Lemma 7.1, Lemma 7.3].

This latter property immediately implies the following integral comparisons:

Proposition 34. Suppose $\delta_{0} \leq \mathfrak{i}(p)$ is sufficiently small with respect to the fundamental constants. Then, for every $0<v<\delta_{0}$ and $\Psi \in \Gamma \mathcal{T} \overline{\mathcal{T}} \mathcal{N}$, we have

$$
\int_{\mathcal{S}_{v}}|\Psi| \simeq v^{2} \int_{\mathbb{S}^{2}} \mid \Psi \|_{(v, \omega)} d \omega .
$$

In particular, for any $1 \leq q, r \leq \infty$, we also have

$$
\|\Psi\|_{L_{t}^{q} L^{r}(\mathcal{N})} \simeq\left\|t_{p}^{\frac{2}{r}} \Psi\right\|_{L_{t}^{q} L_{\omega}^{r}(\mathcal{N})}, \quad\|\Psi\|_{L^{q}(\mathcal{N})} \simeq\|\Psi\|_{L_{t}^{q} L^{q}(\mathcal{N})} \simeq\left\|t_{p}^{\frac{2}{q}} \Psi\right\|_{L_{t}^{q} L_{\omega}^{q}(\mathcal{N})} .
$$

In other words, the natural iterated $L_{t}^{q} L^{r}$-norms are equivalent to the $(v, \omega)$ parametrized norms. The latter representation is especially important, since it is expressed as an integral over the product $\left(0, \delta_{0}\right) \times \mathbb{S}^{2}$, and hence the order of integration can be easily reversed. These reversed $L_{\omega}^{r} L_{t}^{p}$-norms will be essential in numerous estimates, especially within the proof of Theorem 33 .

The regularity of the metric $\bar{\lambda}$, as described in [21, Lemma 7.3], can be used to derive uniform first and second-order Sobolev estimates in a process analogous to that of Proposition [17. The results are given below:

Proposition 35. Suppose $\delta_{0} \leq \mathfrak{i}(p)$ is sufficiently small with respect to the fundamental constants. Then, for every $0<v<\delta_{0}, 2<r<\infty$, and $\Psi \in \Gamma \mathcal{T} \mathcal{N}$,

$$
\begin{aligned}
\|\Psi\|_{L^{\infty}\left(\mathcal{S}_{v}\right)} & \lesssim v^{1-\frac{2}{r}}\|\not \nabla \Psi\|_{L^{r}\left(\mathcal{S}_{v}\right)}+v^{-\frac{2}{r}}\|\Psi\|_{L^{r}\left(\mathcal{S}_{v}\right)}, \\
\|\Psi\|_{L^{r}\left(\mathcal{S}_{v}\right)} & \lesssim\|\not \nabla \Psi\|_{L^{2}\left(\mathcal{S}_{v}\right)}^{1-\frac{2}{r}}\|\Psi\|_{L^{2}\left(\mathcal{S}_{v}\right)}^{\frac{2}{r}}+v^{\frac{2}{r}-1}\|\Psi\|_{L^{2}\left(\mathcal{S}_{v}\right)} \\
& \lesssim v^{\frac{2}{r}}\|\not \nabla \Psi\|_{L^{2}\left(\mathcal{S}_{v}\right)}+v^{\frac{2}{r}-1}\|\Psi\|_{L^{2}\left(\mathcal{S}_{v}\right)}, \\
\|\Psi\|_{L^{\infty}\left(\mathcal{S}_{v}\right)} & \lesssim\left\|\not \nabla^{2} \Psi\right\|_{L^{2}\left(\mathcal{S}_{v}\right)}^{\frac{1}{2}}\|\Psi\|_{L^{2}\left(\mathcal{S}_{v}\right)}^{\frac{1}{2}}+v^{-1}\|\Psi\|_{L^{2}\left(\mathcal{S}_{v}\right)} \\
& \lesssim v\left\|\nabla^{2} \Psi\right\|_{L^{2}\left(\mathcal{S}_{v}\right)}+v^{-1}\|\Psi\|_{L^{2}\left(\mathcal{S}_{v}\right)} .
\end{aligned}
$$

In the first two estimates, the constants of the inequalities also depend on $r$.

Proof. See [21, Sec. 2.1, Sec. 7.2].

Remark. The powers of $v$ present throughout the estimates of Proposition 35 are consequences of the fact that the uniformities of this setting occur with respect to the rescaled metrics $\bar{\lambda}$ rather than the induced metric $\lambda$. 
In addition to the Sobolev inequalities of Proposition 35, which are estimates on the individual $\mathcal{S}_{v}$ 's, we have the following first-order "null Sobolev" inequality, which is in contrast a Sobolev-type estimate on all of $\mathcal{N}$.

Proposition 36. Suppose $\delta_{0} \leq \mathfrak{i}(p)$ is sufficiently small with respect to the fundamental constants. Then, for any $\Psi \in \Gamma \mathcal{T} \mathcal{N}$, the following estimate holds:

$$
\left\|t_{p}^{\frac{1}{2}} \Psi\right\|_{L_{\omega}^{4} L_{t}^{\infty}(\mathcal{N})}+\|\Psi\|_{L^{6}(\mathcal{N})}+\left\|t_{p}^{\frac{1}{2}} \Psi\right\|_{L_{\omega}^{2} L_{t}^{\infty}(\mathcal{N})} \lesssim\|\Psi\|_{\mathcal{H}^{1}(\mathcal{N})} .
$$

As a consequence, the following estimates hold for any $2 \leq d \leq \infty$ :

$$
\|\Psi\|_{L_{t}^{\infty} L^{4}(\mathcal{N})} \lesssim\|\Psi\|_{\mathcal{H}^{1}(\mathcal{N})}, \quad\left\|t_{p}^{-\frac{1}{2}-\frac{1}{d}} \Psi\right\|_{L_{t_{p}}^{d} L^{2}(\mathcal{N})} \lesssim\|\Psi\|_{\mathcal{H}^{1}(\mathcal{N})} .
$$

Proof. See [21, Lemma 7.8].

In Theorem 33 we have sufficiently controlled the Ricci coefficients $\chi$ and $\zeta$. The

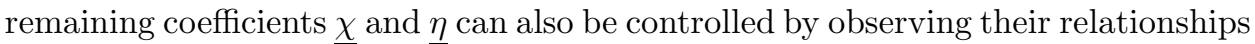
with $\chi, \zeta$, and the quantities $k$ and $n$ derived from the time foliation.

Proposition 37. Suppose $\delta_{0} \leq \mathfrak{i}(p)$ is sufficiently small with respect to the fundamental constants. Then, the following bounds hold on $\mathcal{N}$ :

$$
\|\underline{\eta}\|_{L^{\infty}(\mathcal{N})}+\|\not \nabla \vartheta\|_{L_{\omega}^{\infty} L_{t}^{2}(\mathcal{N})}+\left\|\vartheta(\operatorname{tr} \underline{\chi})+\frac{2}{t_{p}}\right\|_{L^{\infty}(\mathcal{N})}+\|\underline{\hat{\chi}}\|_{L_{\omega}^{\infty} L_{t}^{2}(\mathcal{N})} \lesssim 1 .
$$

Proof. The $L^{\infty}$-bound for $\underline{\eta}$ follows immediately from (40) and (55), and the trace bound for $\not \nabla$ follows from this due to (54) and (109). To handle the estimates for $\underline{\chi}$, we resort to the identity (56), from which one can derive the bounds

$$
\left|\vartheta(\operatorname{tr} \underline{\chi})+\frac{2}{t_{p}}\right| \lesssim 1+\left|\vartheta(\operatorname{tr} \chi)-\frac{2}{t_{p}}\right|, \quad|\underline{\hat{\chi}}| \lesssim 1+|\hat{\chi}|
$$

on $\mathcal{N} .27$ The desired estimates for $\underline{\chi}$ now follow from (109) and (110).

Remark. It is also possible to obtain estimates for $\underline{\eta}, \not \nabla \vartheta$, and $\underline{\chi}$ in the $\mathcal{H}^{1}$-norm analogous to those for $\chi$ and $\zeta$. Such estimates are in fact important within the proof of Theorem 33 itself. However, we will not need these results in this paper.

Remark. If $\mathcal{K}$ is the Gauss curvatures of the $\mathcal{S}_{v}$ 's, then the quantity $\mathcal{K}-\vartheta^{-2} t_{p}^{-2}$ also satisfies estimates in both the $L^{2}(\mathcal{N})$-norm and the $L_{t}^{\infty} H^{-1 / 2}(\mathcal{N})$-norm. The latter bound is particularly essential to the proof of Theorem 33, since it validates numerous elliptic estimates on the $\mathcal{S}_{v}$ 's that play a fundamental role in the proof; see [21, Sec. 2.3, Sec. 7.2]. Since these bounds play no role outside of the proof of Theorem 33, however, we omit serious discussions of this topic within this paper.

In the remainder of this section, we will sketch the proof of Theorem 33 . For the sake of clarity, this will be divided into multiple steps. Again, the reader is referred to 21] for a detailed account of the proof.

\footnotetext{
${ }^{27}$ See [21 Cor. 4.3].
} 
6.5. Proof Outline I: Bootstrap Assumptions. The main argument is a "bootstrap", in which we assume much of what we are trying to prove and proceed to derive even better estimates. We can then establish via a standard continuity argument that these estimates hold even without the assumptions.

To be more specific, we define the following two conditions, which correspond to the conclusions of Theorem [33. Let $p \in M_{+}$, and let $0<\delta_{0} \leq 1$ and $\Delta_{0} \geq 1$ be fixed constants, whose values will be determined later.

- $(\mathbf{N 0})_{p, \delta_{0}, \Delta_{0}}$ : If $\delta=\min \left(\delta_{0}, t(p)-\tau_{0}, \mathfrak{i}(p)\right)$ and $\mathcal{N}_{0}=\mathcal{N}^{-}(p ; \delta)$, then

$$
\begin{aligned}
\Delta_{0} \geq \| & \vartheta(\operatorname{tr} \chi)-\frac{2}{t_{p}}\left\|_{L_{\omega}^{\infty} L_{t}^{2}\left(\mathcal{N}_{0}\right)}+\right\| \vartheta(\operatorname{tr} \chi)-\frac{2}{t_{p}} \|_{\mathcal{H}^{1}\left(\mathcal{N}_{0}\right)} \\
& +\|\hat{\chi}\|_{L_{\omega}^{\infty} L_{t}^{2}\left(\mathcal{N}_{0}\right)}+\|\hat{\chi}\|_{\mathcal{H}^{1}\left(\mathcal{N}_{0}\right)}+\|\zeta\|_{L_{\omega}^{\infty} L_{t}^{2}\left(\mathcal{N}_{0}\right)}+\|\zeta\|_{\mathcal{H}^{1}\left(\mathcal{N}_{0}\right)} \\
& +\left\|\mathcal{K}-\frac{1}{\vartheta^{2} t_{p}^{2}}\right\|_{L_{t}^{\infty} H^{-\frac{1}{2}}\left(\mathcal{N}_{0}\right)}+\left\|\mathcal{K}-\frac{1}{\vartheta^{2} t_{p}^{2}}\right\|_{L^{2}\left(\mathcal{N}_{0}\right)} .
\end{aligned}
$$

- $(\mathbf{N} 1)_{p, \delta_{0}, \Delta_{0}}$ : If $\delta=\min \left(\delta_{0}, t(p)-\tau_{0}\right)$, then $\mathfrak{i}(p)>\delta$, and the inequality (111) holds for the null cone segment $\mathcal{N}_{0}=\mathcal{N}^{-}(p ; \delta)$.

Note that the $(\mathbf{N} 1)_{p, \delta_{0}, \Delta_{0}}$ condition implies the $(\mathbf{N} 0)_{p, \delta_{0}, \Delta_{0}}$ condition. Furthermore, the conclusions of Theorem 33 imply that the $(\mathbf{N} 1)_{q, \delta_{0}, \Delta_{0}}$ condition holds over all $q \in M_{+}$for some $0<\delta_{0} \leq 1$ and $\Delta_{0} \geq 1$ depending only on the fundamental constants. The $(\mathbf{N} \mathbf{0})_{p, \delta_{0}, \Delta_{0}}$ condition, on the other hand, corresponds to the conclusions of Theorem 33 except the null injectivity radius bound.

We begin the proof of Theorem 33 by assuming the following:

The conditions (N0) $)_{q, \delta_{0}, \Delta_{0}}$ holds for every $q \in M_{+}$, with the associated parameters $0<\delta_{0} \leq 1$ "sufficiently small" and $\Delta_{0} \geq 1$ "sufficiently large", both depending only on the fundamental constants.

This is the explicit bootstrap assumption we use for our proof. The precise requirements for $\delta_{0}$ and $\Delta_{0}$ in our bootstrap assumption is determined within the proof. Our goal is then to show the following estimates:

$$
\text { The conditions (N0) })_{q, \delta_{0}, \Delta_{0} / 2} \text { hold for every } q \in M_{+} \text {. }
$$

Once we establish this, we can conclude that the conditions (N0) $)_{q, \delta_{0}, \Delta_{0}}$ hold even without the bootstrap assumptions. 28 This is essentially the bootstrap argument.

To carry out this process, we require numerous auxiliary estimates, by which we mean estimates that are consequences of the $(\mathbf{N 0})_{p, \delta_{0}, \Delta_{0}}$ condition. This is fundamental to the proof, as many of these auxiliary estimates imply sufficient regularity on the $\mathcal{S}_{v}$ 's so that the relevant analysis tools can be applied.

The primary examples of auxiliary estimates are the properties described in Section 6.4 Note that these properties, in particular the bounds and comparisons of Propositions 34][37, depend only on the (N0) $)_{p, \delta_{0}, \Delta_{0}}$ condition and not on the validity of Theorem 33 itself. For example, the Sobolev estimates of Propositions 35 and 36 are vitally important for various basic geometric Littlewood-Paley estimates.

Another important class of auxiliary estimates are the symmetric Hodge-elliptic estimates on the $\mathcal{S}_{v}$ 's. These arguments are essentially variations of those originally introduced in [7, Ch. 2]. First, we define the following operators:

\footnotetext{
${ }^{28}$ We of course also need the initial value results of Proposition 11
} 
- Given a horizontal 1-form $\xi \in \underline{\mathfrak{X}}(\mathcal{N})$, we define

$$
\mathcal{D}_{1} \xi \in C^{\infty}(\mathcal{N}) \times C^{\infty}(\mathcal{N}), \quad \mathcal{D}_{1} \xi=\left(\lambda^{a b} \nabla_{a} \xi_{b}, \epsilon^{a b} \not_{a} \xi_{b}\right),
$$

where $\epsilon$ is the associated volume forms on the $\mathcal{S}_{v}$ 's.

- Given a symmetric traceless $\xi \in \underline{T}^{2} \mathcal{N}$, we define

$$
\mathcal{D}_{2} \xi \in \underline{\mathfrak{X}}(\mathcal{N}), \quad \mathcal{D}_{2} \xi_{a}=\lambda^{b c} \not_{b} \xi_{c a} .
$$

- Given $\left(\xi_{1}, \xi_{2}\right) \in C^{\infty}(\mathcal{N}) \times C^{\infty}(\mathcal{N})$, we define

$$
\mathcal{D}_{1}^{*} \xi \in \underline{\mathfrak{X}}(\mathcal{N}), \quad \mathcal{D}_{1}^{*}\left(\xi_{1}, \xi_{2}\right)_{a}=-\not_{a} \xi_{1}-\epsilon_{a}{ }^{b} \not_{b} \xi_{2} .
$$

The notation $\mathcal{D}_{1}^{*}$ is justified by the fact that $\mathcal{D}_{1}^{*}$ is the $L^{2}$-adjoint of $\mathcal{D}_{1}$.

Here is where the $L_{t}^{\infty} H^{-1 / 2}$-estimate for $\mathcal{K}-\vartheta^{-2} t_{p}^{-2}$ becomes essential. From this assumption, we can prove that $\mathcal{D}_{1}$ and $\mathcal{D}_{2}$ are one-to-one, and hence we can define via projections their $L^{2}$-bounded "inverses" $\mathcal{D}_{1}^{-1}$ and $\mathcal{D}_{2}^{-1}$. Although $\mathcal{D}_{1}^{*}$ clearly fails to be injective, we can still define a viable "inverse" $\left(\mathcal{D}_{1}^{*}\right)^{-1}$ by "modding out" constant functions and mapping to the canonical element with zero mean. For detailed explanations of these procedures, consult [21, Sec. 2.3]. The upshot of this development is the following set of auxiliary Hodge-elliptic estimates:

Lemma 38. If the (No) $)_{p, \delta_{0}, \Delta_{0}}$ condition holds for sufficiently small $\delta_{0}$ (with respect to $\Delta_{0}$ and the fundamental constants), then the following estimates hold:

$$
\left\|\not \nabla \mathcal{D}^{-1} \xi\right\|_{L^{2}\left(\mathcal{S}_{v}\right)}+v^{-1}\left\|\mathcal{D}^{-1} \xi\right\|_{L^{2}\left(\mathcal{S}_{v}\right)} \lesssim\|\xi\|_{L^{2}\left(\mathcal{S}_{v}\right)}, \quad \mathcal{D} \in\left\{\mathcal{D}_{1}, \mathcal{D}_{2}, \mathcal{D}_{1}^{*}\right\}
$$

Proof. See [21, Prop. 2.28, (7.29)].

From Lemma 38 and Proposition 35, we can obtain additional estimates for the inverse Hodge operators; see [21, Prop. 2.29, (7.30)] for details.

6.6. Proof Outline II: The Sharp Trace Estimate. The next batch of auxiliary estimates are the basic geometric Littlewood-Paley estimates and the corresponding Besov estimates on both $\mathcal{N}$ and the $\mathcal{S}_{v}$ 's. These were listed in [21, Sec. 7.3], while much of the background material is within [21, Ch. 2].

The main issue is that we are required to make use of Besov norms within the proof of Theorem 33. Recall that in $\mathbb{R}^{2}$, although the Sobolev embedding $H^{1} \hookrightarrow L^{\infty}$ fails, the corresponding Besov space $B_{2,1}^{1}$, on the other hand, does embed into $L^{\infty}$. In fact, we will need an analogous Besov embedding property on the $\mathcal{S}_{v}$ 's. This will be necessary in particular for the $L_{\omega}^{\infty} L_{t}^{2}$-type estimates for $\hat{\chi}$ and $\zeta$.

To begin with, one must make sense of Besov norms. This task was accomplished in 13 via the construction of a geometric tensorial Littlewood-Paley (abbreviated $L-P)$ theory. 29 In terms of our current setting, we can systematically define "L-P projections" $P_{k}, k \in \mathbb{Z}$, which are used to dyadically decompose horizontal tensor fields on $\mathcal{N}$. These $P_{k}$ 's are defined explicitly using the heat flow operators intrinsic to the $\mathcal{S}_{v}$ 's; see [13, Sec. 5] and [21, Sec. 2.2] for the exact definitions. 30

One can proceed to show that these geometric L-P operators satisfy many of the same properties as the classical L-P projections on Euclidean spaces.

Lemma 39. Let $\Psi \in \Gamma \underline{\mathcal{T}} \mathcal{N}, k \geq 0$, and $0<v<\delta_{0}$.

\footnotetext{
${ }^{29}$ See also [21, Sec. 2.2] for additional remarks and clarifications on this construction.

${ }^{30}$ The idea of constructing Littlewood-Paley theories from the heat flow, or more generally from diffusion semigroups or martingales, originated from 23 .
} 
- The following estimate holds for each $1 \leq p \leq \infty$ :

$$
\left\|P_{k} \Psi\right\|_{L^{2}\left(\mathcal{S}_{v}\right)} \lesssim\|\Psi\|_{L^{2}\left(\mathcal{S}_{v}\right)}
$$

- The following "finite band" estimates hold for each $1 \leq p \leq \infty$ :

$$
\begin{aligned}
\left\|\Delta P_{k} \Psi\right\|_{L^{p}\left(\mathcal{S}_{v}\right)}=\left\|P_{k} \Delta \Psi\right\|_{L^{p}\left(\mathcal{S}_{v}\right)} & \lesssim 2^{2 k}\|\Psi\|_{L^{p}\left(\mathcal{S}_{v}\right)}, \\
\left\|P_{k} \Psi\right\|_{L^{p}\left(\mathcal{S}_{v}\right)} & \lesssim 2^{-2 k}\|\Delta \Psi\|_{L^{p}\left(\mathcal{S}_{v}\right)} .
\end{aligned}
$$

- The following "finite band" estimates hold:

$$
\begin{aligned}
\left\|\not \nabla P_{k} \Psi\right\|_{L^{2}\left(\mathcal{S}_{v}\right)}+\left\|P_{k} \not \nabla \Psi\right\|_{L^{2}\left(\mathcal{S}_{v}\right)} & \lesssim 2^{k}\|\Psi\|_{L^{2}\left(\mathcal{S}_{v}\right)}, \\
\left\|P_{k} \Psi\right\|_{L^{2}\left(\mathcal{S}_{v}\right)} & \lesssim 2^{-k}\|\not \nabla \Psi\|_{L^{2}\left(\mathcal{S}_{v}\right)} .
\end{aligned}
$$

- If the (No) $)_{p, \delta_{0}, \Delta_{0}}$ condition holds for sufficiently small $\delta_{0}$ (with respect to $\Delta_{0}$ and the fundamental constants), then the following "weak Bernstein inequalities" hold for any $2 \leq p<\infty$ and $1<q \leq 2$ :

$$
\left\|P_{k} \Psi\right\|_{L^{p}\left(\mathcal{S}_{v}\right)} \lesssim 2^{\left(1-\frac{2}{p}\right) k}\|\Psi\|_{L^{2}\left(\mathcal{S}_{v}\right)}, \quad\left\|P_{k} \Psi\right\|_{L^{2}\left(\mathcal{S}_{v}\right)} \lesssim 2^{\left(\frac{2}{q}-1\right) k}\|\Psi\|_{L^{q}\left(\mathcal{S}_{v}\right)} .
$$

Proof. See [13].

In particular, similar to the classical L-P projections, the $P_{k}$ 's convert covariant derivatives into multiplication by a scaling factor (in the weaker sense of $L^{p}$-norms). Several other basic properties for the $P_{k}$ 's can be found in [13, 21.

We can now define (geometric tensorial) Besov norms using the operators $P_{k}$. The Besov-type norms we will need for Theorem 33 are the following:

$$
\begin{aligned}
\|\Psi\|_{\mathcal{B}^{0}(\mathcal{N})} & =\sum_{k \geq 0}\left\|P_{k} \Psi\right\|_{L_{t}^{\infty} L^{2}(\mathcal{N})}+\|\Psi\|_{L_{t}^{\infty} L^{2}(\mathcal{N})}, \\
\|\Psi\|_{\mathcal{P}^{0}(\mathcal{N})} & =\sum_{k \geq 0}\left\|P_{k} \Psi\right\|_{L^{2}(\mathcal{N})}+\|\Psi\|_{L^{2}(\mathcal{N})} .
\end{aligned}
$$

For example, one can prove the following sharp Besov embedding estimate:

$$
\|\phi\|_{L^{\infty}(\mathcal{N})} \lesssim\|\not \nabla \phi\|_{\mathcal{B}^{0}(\mathcal{N})}+\left\|t_{p}^{-1} \phi\right\|_{L_{t}^{\infty} L^{2}(\mathcal{N})}, \quad \phi \in C^{\infty}(\mathcal{N})
$$

Remark. In fact, one of the main reasons why this line of reasoning is so highly technical is because the inequality (116) and other sharp $L^{\infty}-L^{2}$ and $L^{2}-L^{1}$ estimates cannot be established for nonscalar tensorial quantities. This is due to the lack of a satisfactory tensorial "Böchner estimate" controlling the second covariant derivative $\not^{2}$ in $L^{2}$-norms by $\not$. The reason for this is that we only have a very weak $L_{t}^{\infty} H^{-1 / 2}$-type control on the Gauss curvatures of the $\mathcal{S}_{v}$ 's.

The primary tool we will require from our Besov norms is the following auxiliary estimate, which we refer to as the "sharp trace theorem".

Lemma 40. Assume the (N0) $)_{p, \delta_{0}, \Delta_{0}}$ condition holds for sufficiently small $\delta_{0}$ and sufficiently large $\Delta_{0}$ (with respect to the fundamental constants). If $\Psi, P, E \in \Gamma \mathcal{I} \mathcal{N}$, and if $\not \nabla \Psi=\mathbb{\nabla}_{t_{p}} P+E$, then the following "sharp trace" estimate holds:

$$
\|\Psi\|_{L_{\omega}^{\infty} L_{t}^{2}(\mathcal{N})} \lesssim\|\Psi\|_{\mathcal{H}^{1}(\mathcal{N})}+\|P\|_{\mathcal{H}^{1}(\mathcal{N})}+\|E\|_{\mathcal{P}^{0}(\mathcal{N})} .
$$

Proof. See [21, Lemma 7.22] and [14, 24]. 31

\footnotetext{
${ }^{31}$ In fact, the first step is an application of (116).
} 
The goal will be to apply Lemma 40 to $\hat{\chi}$ and $\zeta$. The proof of Lemma 40 requires an extensive number of Littlewood-Paley decompositions, integrations by parts, and Besov estimates. This is a significant technical undertaking in its own right; in fact, all of 14 is dedicated to establishing these estimates. The main ingredient of this proof is the "bilinear trace theorems", in which one bounds integrals along null generators of bilinear tensorial quantities; these were stated and proved for the vacuum case in [14, Thm. 4.9] and [24, Prop. 5.4]. For the E-S and E-M cases, the proofs of these estimates remain essentially unchanged; see [21, Lemma 7.20].

6.7. Proof Outline III: The Null Injectivity Radius. Another one of the most important auxiliary estimates is that of bounding the null injectivity radii from below. Such an estimate would state roughly the following:

Lemma 41. Assume the bootstrap assumptions, i.e., that (N0) $)_{q, \delta_{0}, \Delta_{0}}$ holds for any $q \in M_{+}$. If $\delta_{0}$ is sufficiently small with respect to the fundamental constants, then the $(\mathbf{N} 1)_{q, \delta_{0}, \Delta_{0}}$ condition also holds for every $q \in M_{+}$. In particular, this implies a uniform lower bound for the null injectivity radii $\mathfrak{i}(q), q \in M_{+}$.

The proof of Lemma 41 (in the E-S and E-M cases) is essentially the same as the vacuum case, originally presented in [16]. Only a slight adaptation of this was required for our settings; this was outlined in [21, Sec. 7.4].

For completeness, we provide a short summary of the argument behind Lemma 41. We must control both the null conjugacy radius $\mathfrak{s}(q)$ and the null cut locus radius $\mathfrak{l}(q)$ of each point $q \in M_{+}$. 32 First, we can control the null conjugacy radii by appealing to the following "breakdown principle" for regular null cones:

A null conjugate point cannot occur along $\mathcal{N}^{-}(p)$ if $\operatorname{tr} \chi$ remains finite.

This is true because uniform control on tr $\chi$ implies control on the volume forms of the $\mathcal{S}_{v}$ 's, and hence the $(v, \omega)$-parametrization of $\mathcal{N}^{-}(p)$ can be shown to be nonsingular. As a result of this, we obtain the following:

Lemma 42. If (No) $)_{p, \delta_{0}, \Delta_{0}}$ holds, then $\mathfrak{s}(p)>\min \left(\mathfrak{l}(p), \delta_{0}\right)$.

Proof. See [21, Lemma 7.23].

It remains to control the null cut locus radii $\mathfrak{l}(q), q \in M_{+}$. The argument for this is identical to that found in [16. The main ingredients are the following:

- The regularity properties of the time foliation, established from the a priori estimates of Section 5. In particular, this includes (40) and Proposition 21.

- The following null cone comparison property:

About any $q \in M$, there exists a sufficiently large "almost Minkowski" coordinate system about $q$, within which the regular past null cone is contained within two Minkowski coordinate cones.

For details, consult [21, Prop. 4.11, Prop. 4.12].

As a result of the above, we can make the following observations:

- The $\mathcal{S}_{v}$ 's must be "comparable" to rescaled Euclidean spheres.

- If the first point of intersection between two null generators occurs before null conjugate points, then the generators must intersect with angle $\pi$. For a proof of this property, see [16, Lemma 3.1].

\footnotetext{
${ }^{32}$ Recall that $\mathfrak{s}(p)$ is the timespan before which there are no past null conjugate points of $p$, while $\mathfrak{l}(p)$ is the timespan before which no two distinct null generators of $\mathcal{N}^{-}(p)$ can intersect.
} 
- If two null generators of $\mathcal{N}^{-}(p)$ are opposite at $p$, then they cannot intersect until after a fixed timespan $\delta_{*}$, which depends on the regularity of the time foliation, and hence on the fundamental constants; see [16. Prop. 3.4].

We can show that the above observations suffice to imply a lower bound on the $\mathfrak{l}(q)$ 's. The idea is roughly the following. Using [16, Prop. 3.5], we can generate a pair of null geodesic segments that intersect at two points $p_{0}, q_{0}$ such that:

- The timespan between $p_{0}$ and $q_{0}$ is at most that of any other pair of null geodesic segments that intersect twice.

- The two segments connecting $p_{0}$ and $q_{0}$ are opposite at both $p_{0}$ and $q_{0}$.

Then, the preceding observations, with the help of Lemma 42, will suffice to control this minimal timespan between $p_{0}$ and $q_{0}$. As a result of this argument, then Lemma 41 is properly established. For the details, see [16, Sec. 3].

As a result, we obtain a lower bound on the null injectivity radii as well as the $(\mathbf{N 1})_{q, \delta_{0}, \Delta_{0}}$ conditions. This will be important in the remainder of the proof of Theorem 33. since it is used to derive trace estimates, which bound quantities on the $\mathcal{S}_{v}$ 's by quantities on the timeslices $\Sigma_{\tau}$; see [21, Prop. 7.3, Cor. 7.4, Cor. 7.5].

Remark. We remark that as stated in Lemma 41, the lower bound for the null injectivity radius is contingent on the bootstrap assumptions. However, after completing the bootstrap argument and hence proving that the $(\mathbf{N O})_{q, \delta_{0}, \Delta_{0}}$ properties hold without preconditions, then we can apply Lemma 41 again. This controls the null injectivity radii without requiring the bootstrap assumptions a priori.

6.8. Proof Outline IV: The Improved Estimates. With the necessary auxiliary estimates established, we now turn to deriving improved estimates for the Ricci coefficients and the Gauss curvatures $\mathcal{K}$. This would complete our main bootstrap argument. The details sketched here are performed in detail in [21, Ch. 8].

The Ricci coefficients are related to each other and to the spacetime curvature $R$ via a family of evolutionary (along null generators) and elliptic identities, known as the structure equations. For the full list of structure equations, see [21, Sec. 3.2]. These are adaptations of the analogous equations for vacuum spacetimes found in 17, 12, 19, 24, 25. In particular, in the nonvacuum settings discussed here, there are numerous additional terms in the structure equations corresponding to the Ricci curvature; of course, these terms vanish in the vacuum setting.

Our desired improved estimates will be a consequence of the forms of these structure equations. Let $\Gamma$ denote one of the quantities bounded by the (N0) $)_{p, \delta_{0}, \Delta_{0}}$ condition, i.e., $\Gamma \leq \Delta_{0}$. Our goal, in general, will be to integrate the various structure equations in order to derive estimates roughly of the form

$$
\Gamma \lesssim \delta_{0}^{\frac{1}{2}} \Delta_{0}^{2}+1
$$

where the constant of the inequality depends only on the fundamental constants. In general, the bound $\delta_{0}^{1 / 2} \Delta_{0}^{2}$ is a consequence of terms in the structure equations that are quadratic (and occasionally cubic) in the Ricci coefficients, while "1" reflects those terms which can be controlled using a priori estimates.

If we choose $\Delta_{0}$ to be sufficiently large with respect to the fundamental constants, and we then choose $\delta_{0}$ to be sufficiently small with respect to $\Delta_{0}$ and the fundamental constants, then we obtain the schematic bound

$$
\Gamma \leq C\left(\delta_{0}^{\frac{1}{2}} \Delta_{0}^{2}+1\right) \leq \frac{\Delta_{0}}{2} .
$$


In particular, this means we have proved the $(\mathrm{N} \mathbf{0})_{p, \delta_{0}, \Delta_{0} / 2}$ condition. As a result, the bootstrap argument would be complete. We will now briefly describe how the above schematic bound can be obtained for the possible values of $\Gamma$.

For example, consider the following structure equation:

$$
\not_{t_{p}}(\operatorname{tr} \chi)=-\frac{1}{2} \vartheta(\operatorname{tr} \chi)^{2}-\vartheta|\hat{\chi}|^{2}-\vartheta R_{44}
$$

This is the Raychaudhuri equation, which is perhaps the most well-known of the structure equations. By integrating a slight variant of the Raychaudhuri equation and taking an $L_{\omega}^{\infty} L_{t}^{2}$-norm of the result, we derive

$$
\left\|\vartheta(\operatorname{tr} \chi)-\frac{2}{t_{p}}\right\|_{L_{\omega}^{\infty} L_{t}^{2}(\mathcal{N})} \lesssim \delta_{0}^{\frac{1}{2}} \Delta_{0}^{2}+1 .
$$

The quantity $\Delta_{0}^{2}$ follows from the bootstrap assumption $\Gamma \leq \Delta_{0}$, while the small factor $\delta_{0}^{1 / 2}$ appears because of the integrations involved. The Ricci curvature term in (117) can be controlled using a priori estimates from the previous section.

We can also control the quantity $\mathbb{}_{t_{p}}\left[\vartheta(\operatorname{tr} \chi)-2 t_{p}^{-1}\right]$ in the $L^{2}(\mathcal{N})$-norm using (117). Similar bounds for the $L^{2}$-norms of $\not_{t_{p}} \hat{\chi}$ and $\not_{t_{p}} \zeta$ can be derived using the corresponding evolution equations for $\hat{\chi}$ and $\zeta$.

At this point, we can also prove analogous estimates for the quantities in the lefthand side of (110) by integrating the appropriate evolutionary structure equations. These estimates will in fact function like the auxiliary estimates:

- These bounds will be essential for completing the bootstrap argument.

- Once the bootstrap argument is completed, and (109) is established, then (110) will immediately follow from (109) and these estimates.

Remark. The evolutionary structure equations are listed in [21, Prop. 3.8].

Next, we turn to the elliptic structure equations, cf. [21, Prop. 3.9], from which we can control $\not \nabla \hat{\chi}$ and $\not \varnothing \zeta$ in the $L^{2}$-norms. These equations are of the forms

$$
\mathcal{D}_{2} \hat{\chi}=\check{R}+\not(\operatorname{tr} \chi)+B, \quad \mathcal{D}_{1} \zeta=\check{R}+\mu+B,
$$

where $\check{R}$ represents certain components of the spacetime Riemann and Ricci curvatures, and $B$ represents lower-order terms. Since the right-hand sides of these elliptic equations can be easily controlled in the $L^{2}(\mathcal{N})$-norm, then direct applications of Lemma 38 yield the desired improved estimates for $\not \nabla \hat{\chi}$ and $\not \nabla \zeta$. These are once again of the familiar form $\Gamma \lesssim \delta_{0}^{1 / 2} \Delta_{0}^{2}+1$.

Furthermore, using the structure equation

$$
\mathcal{K}=-\frac{1}{4}(\operatorname{tr} \chi)(\operatorname{tr} \underline{\chi})+\frac{1}{2} \hat{\chi}^{a b} \underline{\hat{\chi}}_{a b}-\frac{1}{4} R_{4343}+\frac{1}{2} R_{a}{ }^{a}+\frac{1}{2} R_{43},
$$

we can proceed to derive the following improved estimates:

$$
\left\|\mathcal{K}-\frac{1}{\vartheta^{2} t_{p}^{2}}\right\|_{L_{t}^{\infty} H^{-\frac{1}{2}}(\mathcal{N})} \lesssim \delta_{0}^{\frac{1}{2}} \Delta_{0}^{2}+1, \quad\left\|\mathcal{K}-\frac{1}{\vartheta^{2} t_{p}^{2}}\right\|_{L^{2}(\mathcal{N})} \lesssim \delta_{0}^{\frac{1}{2}} \Delta_{0}^{2}+1 .
$$

The latter bound is straightforward, but the former requires much more effort due to the presence of the fractional Sobolev $H^{-1 / 2}$-norm. 33 Indeed, for the $L_{t}^{\infty} H^{-1 / 2}$ bound, we need a technical estimate for the commutator $\left[\Lambda^{-1 / 2}, \mathbb{\nabla}_{t_{p}}\right]$, where $\Lambda^{-1 / 2}$

\footnotetext{
${ }^{33}$ See [13 and 21 Sec. 2.2] for the definition of the $H^{s}$-norms.
} 
is the fractional Sobolev operator on the $\mathcal{S}_{v}$ 's, defined in the standard fashion in terms of $\not \Delta$. For details, see [21, Lemma 8.8], as well as [12, 24].

It remains only to establish improved estimates for the $L_{\omega}^{\infty} L_{t}^{2}$-norms of $\hat{\chi}$ and $\zeta$. As mentioned earlier, the strategy is to utilize Lemma 40 . In particular, we must show that $\not \nabla \hat{\chi}$ and $\not \nabla \zeta$ have decompositions of the form $\not_{t_{p}} P+E$, where the quantities $P$ and $E$ have sufficient bounds, as specified in Lemma 40, This is a priori not obvious; the argument in fact spans nearly all of [21, Sec. 8.2-8.4].

The main ideas and steps for deriving these decompositions are the following:

- First, since the structure equations only provide relations for $\mathcal{D}_{2} \hat{\chi}$ and $\mathcal{D}_{1} \zeta$, not for $\not{\nabla} \hat{\chi}$ and $\not \nabla \zeta$, we must apply the inverse Hodge operators, e.g.,

$$
\not \nabla \hat{\chi}=\not \nabla \mathcal{D}_{2}^{-1} \mathcal{D}_{2} \hat{\chi}=\not \nabla \mathcal{D}_{2}^{-1} \check{R}+\not \nabla \mathcal{D}_{2}^{-1} \not \nabla(\operatorname{tr} \chi)+\not \nabla \mathcal{D}_{2}^{-1} B
$$

- In order to generate the components $\mathbb{D}_{t_{p}} P$ in the desired decomposition, we must take advantage of additional relations satisfied by $\check{R}$. For instance, for $\zeta$, this is a "null Bianchi equation" of the form

$$
\mathcal{D}_{1}^{*} \check{R}=\not_{t_{p}} \check{R}+D,
$$

where $D$ denotes lower-order terms. From this, we see that we will also need to work with the "inverse" operator $\left(\mathcal{D}_{1}^{*}\right)^{-1}$.

- Next, combining the previous two steps, we see that we must commute $\not_{t_{p}}$ with $\not \nabla$ and the inverse Hodge operators. In particular, this requires multiple commutator estimates in both Besov and lower-order norms.

- Unfortunately, there exist "bad" commutator terms which cannot be adequately controlled. In fact, these terms must themselves be further decomposed by adopting arguments analogous to the above, which yields an additional level of "good" and "bad" terms. 34 This ultimately results in an infinite sequence of decompositions, which can be shown to converge. Only in this fashion can the "bad" commutator terms be treated.

- After the infinite sequence of renormalizations, we finally obtain the desired decompositions for $\not \nabla \hat{\chi}$ and $\not \nabla \zeta$. We must also show that components of these decompositions can be controlled as required by Lemma 40.

Once all of the above is complete, then Lemma 40 yields the desired improved estimates for both $\hat{\chi}$ and $\zeta$ in the $L_{\omega}^{\infty} L_{t}^{2}$-norm.

Remark. For the most part, the Ricci curvature terms in the structure equations (which are unique to the nonvacuum settings) are harmless, as they generally have better bounds than the Riemann curvature terms due to (60) and Proposition 24. The main exception to this is for the above decomposition argument, for which the higher-order Ricci curvature terms require their own unique decompositions. In particular, for the E-M case, we must make use of analogous null Bianchi equations for components of F. For details, see [21, Sec. 8.3].

As a result of all of the above, we obtain the improved estimates $\Gamma \leq \Delta_{0} / 2$, which proves the $(\mathbf{N 0})_{q, \delta_{0}, \Delta_{0} / 2}$ condition for all $q \in M_{+}$and hence completes the bootstrap process. By a standard continuity argument, we obtain that the (N0 $)_{q, \delta_{0}, \Delta_{0}}$ condition holds for all $q \in M_{+}$without the a priori bootstrap assumption.

Finally, by Lemma 41, the conditions $(\mathbf{N 1})_{q, \delta_{0}, \Delta_{0}}$ also hold for all $q \in M_{+}$ without prior assumptions. This implies the desired past null injectivity radius

\footnotetext{
${ }^{34}$ The "good" terms will be contribute to both $\not_{t_{p}} P$ and $E$.
} 
bounds, along with (109). The remaining estimates (110) now follow from (109), as discussed before. This completes the proof of Theorem 33 .

\section{Higher-Order Energy Estimates}

Now that we have established both a priori estimates and local null geometry estimates, we can proceed to derive higher-order energy estimates and then complete the proof of Theorem [6. Our main goal is higher-order $L^{2}$-estimates on $k, \mathcal{R}$, and the horizontal formulation of the matter field; these are the objects represented by the quantities $\mathfrak{K}(\tau), \mathfrak{R}(\tau), \mathfrak{f}(\tau)$ in (44)-(46). Like for the a priori estimates, we obtain these by first deriving higher-order energy inequalities for the spacetime quantities $R$ and the matter field $\Phi$, and then deriving elliptic estimates.

Unlike for the a priori estimates, we will also require $L^{\infty}$-bounds for $R$ and the matter field ( $D^{2} \phi$ in the E-S case, $D F$ in the E-M case) in order to complete the higher-order energy estimates. For this task, we will make use of the generalized representation formula for covariant tensorial wave equations discussed in [21, 22. This is an extension of the formula of S. Klainerman and I. Rodnianski in 15, which was applied in an analogous fashion in the E-V case. However, the formula of [15] fails in the E-M case due to additional first-order terms.

7.1. Covariant Wave Relations. We have previously made use of the fact that the matter field ( $D \phi$ in the E-S case, and $F$ in the E-M case) satisfies a covariant wave equation. We now list similar wave equations for $R, D R$, and higher derivatives of the matter field. We can then derive energy inequalities from these relations using the same EMT techniques as in Propositions 21 and 24.

To begin with, by taking the divergence of the Bianchi identities (85) for $R$ and then commuting derivatives, we obtain the covariant wave equation

$$
\square_{g} R_{\alpha \beta \gamma \delta}=-D_{\alpha}{ }^{\mu} R_{\beta \mu \gamma \delta}-D_{\beta}{ }^{\mu} R_{\mu \alpha \gamma \delta}+R_{\alpha}{ }^{\mu}[R]_{\beta \mu \gamma \delta}+R_{\beta}{ }^{\mu}[R]_{\mu \alpha \gamma \delta} .
$$

Applying either (25) or (26), depending on the matter model at hand, and expanding the terms quadratic in $R$, we obtain the nonlinear wave equation

$$
\begin{aligned}
\square_{g} R & =\tilde{d}^{2} \operatorname{Ric}+R * R, \\
(R * R)_{\alpha \beta \gamma \delta} & =\mathfrak{A}_{\alpha \beta}\left\{R_{\alpha}{ }^{\lambda} R_{\lambda \beta \gamma \delta}\right\}+2 R_{\alpha}{ }^{\lambda}{ }^{\mu}{ }^{\mu} R_{\mu \lambda \gamma \delta}-2 \mathfrak{A}_{\alpha \beta}\left\{R_{\alpha}{ }^{\mu}{ }^{\lambda}{ }^{\lambda} R_{\beta \mu \delta \lambda}\right\} .
\end{aligned}
$$

The term $\tilde{d}^{2}$ Ric has the following expansions in the E-S and E-M cases, respectively:

$$
\begin{aligned}
\left(\tilde{d}^{2} \mathrm{Ric}\right)_{\alpha \beta \gamma \delta}=- & 2 \mathfrak{A}_{\alpha \beta}\left\{D_{\alpha \gamma} \phi D_{\beta \delta} \phi\right\}-\mathfrak{A}_{\alpha \beta} \mathfrak{A}_{\gamma \delta}\left\{D_{\lambda} \phi D_{\gamma} \phi R_{\alpha \delta \beta}{ }^{\lambda}\right\}, \\
\left(\tilde{d}^{2} \mathrm{Ric}\right)_{\alpha \beta \gamma \delta}= & \mathfrak{A}_{\alpha \beta}\left\{F_{\alpha}{ }^{\mu} D_{\beta \mu} F_{\gamma \delta}\right\}+\mathfrak{A}_{\gamma \delta}\left\{F_{\gamma}{ }^{\mu} D_{\delta \mu} F_{\alpha \beta}\right\}-\mathfrak{A}_{\alpha \beta} \mathfrak{A}_{\gamma \delta}\left\{D_{\alpha} F_{\gamma \mu} D_{\delta} F_{\beta}{ }^{\mu}\right\} \\
& +D^{\mu} F_{\alpha \beta} D_{\mu} F_{\gamma \delta}+\mathfrak{A}_{\alpha \beta} \mathfrak{A}_{\gamma \delta}\left\{F^{\lambda \mu} F_{\gamma \mu} R_{\alpha \lambda \beta \delta}+F_{\gamma \mu} F_{\alpha \lambda} R_{\beta \delta}{ }^{\mu \lambda}\right\} \\
& -\frac{1}{2} \mathfrak{A}_{\alpha \beta} \mathfrak{A}_{\gamma \delta}\left\{g_{\alpha \gamma}\left(F^{\mu \lambda} D_{\beta \delta} F_{\mu \lambda}+D_{\beta} F_{\mu \lambda} D_{\delta} F^{\mu \lambda}\right)\right\},
\end{aligned}
$$

Recall that $\mathfrak{A}$, defined in (7), denotes anti-symmetrizations of the indices.

In the E-S case, by differentiating (86) and commuting derivatives, we can derive a wave equation for $D^{2} \phi$. More specifically, we can compute

$$
\begin{aligned}
\square_{g} D_{\alpha \beta} \phi= & D_{\alpha \mu \beta}^{\mu} \phi+D^{\mu}\left(R_{\beta}{ }^{\lambda}{ }_{\mu \alpha} D_{\lambda} \phi\right) \\
= & D_{\alpha} \square_{g} D_{\beta} \phi+R_{\mu}{ }^{\lambda \mu}{ }_{\alpha} D_{\lambda \beta} \phi+R_{\beta}{ }^{\lambda \mu}{ }_{\alpha} D_{\mu \lambda} \phi \\
& \quad+D^{\mu} R_{\mu \alpha \beta}{ }^{\lambda} D_{\lambda} \phi+R_{\mu \alpha \beta}{ }^{\lambda} D^{\mu}{ }_{\lambda} \phi .
\end{aligned}
$$


By (25) and (86), then

$$
\begin{aligned}
\square_{g} D_{\alpha \beta} \phi=2 D^{\lambda} \phi D_{\lambda} \phi D_{\alpha \beta} \phi+D_{\alpha} \phi D^{\lambda} \phi D_{\beta \lambda} \phi \\
+D_{\beta} \phi D^{\lambda} \phi D_{\alpha \lambda} \phi-2 R_{\alpha}{ }^{\mu}{ }^{\lambda}{ }^{\lambda} D_{\mu \lambda} \phi .
\end{aligned}
$$

By a similar process, in the E-M case, we have the wave relation

$$
\begin{aligned}
\square_{g} D_{\gamma} F_{\alpha \beta}=2 & F^{\lambda \mu} D_{\gamma} R_{\alpha \lambda \mu \beta}+\mathfrak{A}_{\alpha \beta}\left\{R_{\alpha}{ }^{\lambda} \gamma^{\mu} D_{\mu} F_{\beta \lambda}\right\}+2 R_{\alpha}{ }^{\lambda \mu}{ }_{\beta} D_{\gamma} F_{\lambda \mu} \\
& +2 F_{\alpha}{ }^{\mu} F_{\beta}{ }^{\lambda} D_{\gamma} F_{\mu \lambda}+2 \mathfrak{A}_{\alpha \beta}\left\{F^{\mu \lambda} F_{\alpha \mu} D_{\gamma} F_{\beta \lambda}\right\} \\
& -F^{\mu \lambda} F_{\alpha \beta} D_{\gamma} F_{\mu \lambda}-\frac{3}{4} F^{\mu \lambda} F_{\mu \lambda} D_{\gamma} F_{\alpha \beta} \\
& -F^{\lambda \mu} F_{\gamma \mu} D_{\lambda} F_{\alpha \beta}-\mathfrak{A}_{\alpha \beta}\left\{F_{\alpha}{ }^{\lambda} F_{\gamma}{ }^{\mu} D_{\mu} F_{\beta \lambda}\right\} \\
& -\mathfrak{A}_{\alpha \beta}\left[F_{\alpha}{ }^{{ }} \mathfrak{A}_{\beta \lambda}\left(F_{\beta}{ }^{\mu} D_{\lambda} F_{\gamma \mu}-\frac{1}{2} g_{\beta \gamma} F^{\mu \nu} D_{\lambda} F_{\mu \nu}\right)\right] .
\end{aligned}
$$

In addition to the above, we will need covariant wave equations for one higher derivative of $R, \phi$, and $F$. Fortunately, we will require only the schematic forms, not the exact equations. In the E-S case, we have

$$
\begin{aligned}
& \square_{g} D R \cong D^{2} \phi \cdot D^{3} \phi+D \phi \cdot D^{2} \phi \cdot R+(D \phi)^{2} \cdot D R+R \cdot D R, \\
& \square_{g} D^{3} \phi \cong(D \phi)^{2} \cdot D^{3} \phi+D \phi \cdot\left(D^{2} \phi\right)^{2}+R \cdot D^{3} \phi+D R \cdot D^{2} \phi .
\end{aligned}
$$

Similarly, in the E-M case,

$$
\begin{aligned}
& \square_{g} D R \cong F \cdot D^{3} F+D F \cdot D^{2} F+F \cdot D F \cdot R+F^{2} \cdot D R+R \cdot D R, \\
& \square_{g} D^{2} F \cong F \cdot D^{2} R+D F \cdot D R+R \cdot D^{2} F+F \cdot(D F)^{2}+F^{2} \cdot D^{2} F .
\end{aligned}
$$

7.2. Energy Inequalities. With the wave relations (118)-(124) in hand, we can now apply general EMT methods to derive preliminary energy inequalities for the curvature and the matter field. Like for the a priori estimates of Proposition 21. we must bound the curvature and the matter field concurrently.

For convenience, in the E-S case, we define the quantities

$$
\mathcal{E}^{2}(\tau)=\|D R\|_{L^{2}\left(\Sigma_{\tau}\right)}^{2}+\left\|D^{3} \phi\right\|_{L^{2}\left(\Sigma_{\tau}\right)}^{2}, \quad \mathcal{E}^{3}(\tau)=\left\|D^{2} R\right\|_{L^{2}\left(\Sigma_{\tau}\right)}^{2}+\left\|D^{4} \phi\right\|_{L^{2}\left(\Sigma_{\tau}\right)}^{2},
$$

while in the E-M case, we define the analogous quantities

$$
\mathcal{E}^{2}(\tau)=\|D R\|_{L^{2}\left(\Sigma_{\tau}\right)}^{2}+\left\|D^{2} F\right\|_{L^{2}\left(\Sigma_{\tau}\right)}^{2}, \quad \mathcal{E}^{3}(\tau)=\left\|D^{2} R\right\|_{L^{2}\left(\Sigma_{\tau}\right)}^{2}+\left\|D^{3} F\right\|_{L^{2}\left(\Sigma_{\tau}\right)}^{2} .
$$

Proposition 43. For any $\tau_{0} \leq \tau_{1}<\tau<t_{1}$, the energy inequalities

$$
\begin{aligned}
& \mathcal{E}^{2}(\tau) \lesssim \mathcal{E}^{2}\left(\tau_{1}\right)+\int_{\tau_{1}}^{\tau}\left(1+\|R\|_{L^{\infty}\left(\Sigma_{\tau^{\prime}}\right)}^{2}+\left\|D^{2} \phi\right\|_{L^{\infty}\left(\Sigma_{\tau^{\prime}}\right)}^{2}\right) d \tau^{\prime}, \\
& \mathcal{E}^{2}(\tau) \lesssim \mathcal{E}^{2}\left(\tau_{1}\right)+\int_{\tau_{1}}^{\tau}\left(1+\|R\|_{L^{\infty}\left(\Sigma_{\tau^{\prime}}\right)}^{2}+\|D F\|_{L^{\infty}\left(\Sigma_{\tau^{\prime}}\right)}^{2}\right) d \tau^{\prime}
\end{aligned}
$$

hold in the $E-S$ and $E-M$ cases, respectively.

Proof. In the E-S case, applying (83) with $\Phi=R$ along with (118) yields

$$
\|D R\|_{L^{2}\left(\Sigma_{\tau}\right)}^{2} \lesssim\|D R\|_{L^{2}\left(\Sigma_{\tau_{1}}\right)}^{2}+\int_{\tau_{1}}^{\tau} \int_{\Sigma_{\tau^{\prime}}}\left(|D R|^{2}+|R|^{4}+\left|D^{2} \phi\right|^{4}+|R|^{2}\right) d \tau^{\prime} .
$$


Similarly, applying (83) with $\Phi=D^{2} \phi$ along with (119) yields

$$
\left\|D^{3} \phi\right\|_{L^{2}\left(\Sigma_{\tau}\right)}^{2} \lesssim\left\|D^{3} \phi\right\|_{L^{2}\left(\Sigma_{\tau_{1}}\right)}^{2}+\int_{\tau_{1}}^{\tau} \int_{\Sigma_{\tau^{\prime}}}\left(\left|D^{3} \phi\right|^{2}+|R|^{2}\left|D^{2} \phi\right|^{2}+\left|D^{2} \phi\right|^{2}\right) d \tau^{\prime} .
$$

In these inequalities, we have used the a priori uniform bounds on $k, n$, and $D \phi$. Summing the above and applying Proposition 21 yields

$$
\mathcal{E}^{2}(\tau) \lesssim \mathcal{E}^{2}\left(\tau_{1}\right)+\int_{\tau_{1}}^{\tau}\left(1+\|R\|_{L^{\infty}\left(\Sigma_{\tau^{\prime}}\right)}^{2}+\left\|D^{2} \phi\right\|_{L^{\infty}\left(\Sigma_{\tau^{\prime}}\right)}^{2}\right) d \tau^{\prime}+\int_{\tau_{1}}^{\tau} \mathcal{E}^{2}\left(\tau^{\prime}\right) d \tau^{\prime} .
$$

Applying Grönwall's inequality results in the desired bound.

The E-M case proceeds analogously; apply (83) with $\Phi=R$ and $\Phi=D F$.

The process behind the proof of Proposition 43 can be repeated for the wave equations (121)-(124) in order to obtain the following:

Proposition 44. For any $\tau_{0} \leq \tau_{1}<\tau<t_{1}$, the energy inequalities

$$
\begin{aligned}
& \mathcal{E}^{3}(\tau) \lesssim \mathcal{E}^{3}\left(\tau_{1}\right)+\int_{\tau_{1}}^{\tau}\left(1+\|R\|_{L^{\infty}\left(\Sigma_{\tau^{\prime}}\right)}^{2}+\left\|D^{2} \phi\right\|_{L^{\infty}\left(\Sigma_{\tau^{\prime}}\right)}^{2}\right)\left[1+\mathcal{E}^{2}\left(\tau^{\prime}\right)\right] d \tau^{\prime}, \\
& \mathcal{E}^{3}(\tau) \lesssim \mathcal{E}^{3}\left(\tau_{1}\right)+\int_{\tau_{1}}^{\tau}\left(1+\|R\|_{L^{\infty}\left(\Sigma_{\tau^{\prime}}\right)}^{2}+\|D F\|_{L^{\infty}\left(\Sigma_{\tau^{\prime}}\right)}^{2}\right)\left[1+\mathcal{E}^{2}\left(\tau^{\prime}\right)\right] d \tau^{\prime}
\end{aligned}
$$

hold in the E-S and E-M cases, respectively.

Proof. In the E-S case, apply (831), (121), and (122) with $\Phi=D R$ and $\Phi=D^{3} \phi$. In the E-M case, apply (83), (123), and (124) with $\Phi=D R$ and $\Phi=D^{2} F$.

Remark. We can obtain identical energy bounds for $D R$ and $D^{2} R$, as well as $D F$, $D^{2} F$, and $D^{3} F$ in the E-M case, using the generalized Maxwell EMTs and (84).

Lastly, we will need a local variant of Proposition 43 involving flux bounds on regular past null cones. This can be derived in the same manner that Proposition 24 was obtained from Proposition 21] Fix $p \in M_{+}$and a time value $\tau_{0} \leq \tau<t(p)$, with $t(p)-\tau<\mathfrak{i}(p)$; we normalize and foliate $\mathcal{N}^{-}(p)$ using $\left.T\right|_{p}$ and $t_{p}$.

Define the sets $\mathcal{N}_{\tau}, \mathcal{I}_{\tau}$, and $\Sigma_{\tau}^{p}$ as in Section [5.5] and define the flux densities

$$
\begin{aligned}
& \rho^{2}(p, \tau)=-Q_{w}[R](T, L)-Q_{w}\left[D^{2} \phi\right](T, L), \\
& \rho^{2}(p, \tau)=-Q_{w}[R](T, L)-Q_{w}[D F](T, L),
\end{aligned}
$$

in the E-S and E-M settings, respectively. We also define the corresponding flux

$$
\mathcal{F}^{2}(p ; \tau)=\int_{\mathcal{N}_{\tau}} \rho^{2}(p, \tau) .
$$

Recall that the notation $Q_{w}[U]$ was defined in Section 5.3 .

Proposition 45. The following hold in the E-S and E-M settings, respectively:

$$
\begin{aligned}
& \mathcal{F}^{2}(p ; \tau) \lesssim \mathcal{E}^{2}(\tau)+\int_{\tau}^{t(p)}\left(1+\|R\|_{L^{\infty}\left(\Sigma_{\tau^{\prime}}\right)}^{2}+\left\|D^{2} \phi\right\|_{L^{\infty}\left(\Sigma_{\tau^{\prime}}\right)}^{2}\right) d \tau^{\prime}, \\
& \mathcal{F}^{2}(p ; \tau) \lesssim \mathcal{E}^{2}(\tau)+\int_{\tau}^{t(p)}\left(1+\|R\|_{L^{\infty}\left(\Sigma_{\tau^{\prime}}\right)}^{2}+\|D F\|_{L^{\infty}\left(\Sigma_{\tau^{\prime}}\right)}^{2}\right) d \tau^{\prime} .
\end{aligned}
$$


Proof. For the E-S case, we apply (88) with $\Phi=R$ and $\Phi=D^{2} \phi$ to obtain

$$
\begin{aligned}
-\int_{\mathcal{N}_{\tau}} Q_{w}[R](T, L) \lesssim & \|D R\|_{L^{2}\left(\Sigma_{\tau}\right)}^{2} \\
& \quad+\int_{\tau}^{t(p)} \int_{\Sigma_{\tau^{\prime}}}\left(|D R|^{2}+|R|^{4}+\left|D^{2} \phi\right|^{4}+|R|^{2}\right) d \tau^{\prime}, \\
-\int_{\mathcal{N}_{\tau}} Q_{w}\left[D^{2} \phi\right](T, L) \lesssim & \left\|D^{3} \phi\right\|_{L^{2}\left(\Sigma_{\tau}\right)}^{2} \\
& +\int_{\tau}^{t(p)} \int_{\Sigma_{\tau^{\prime}}}\left(\left|D^{3} \phi\right|^{2}+|R|^{2}\left|D^{2} \phi\right|^{2}+\left|D^{2} \phi\right|^{2}\right) d \tau^{\prime} .
\end{aligned}
$$

Summing the above bounds and applying Proposition 21, we obtain

$$
\mathcal{F}^{2}(p ; \tau) \lesssim \mathcal{E}^{2}(\tau)+\int_{\tau}^{t(p)}\left[1+\|R\|_{L^{\infty}\left(\Sigma_{\tau^{\prime}}\right)}^{2}+\left\|D^{2} \phi\right\|_{L^{\infty}\left(\Sigma_{\tau^{\prime}}\right)}^{2}+\mathcal{E}^{2}\left(\tau^{\prime}\right)\right] d \tau^{\prime} .
$$

The proof is completed by applying Proposition 43 .

The E-M case is analogous, except we apply (88) with $\Phi=R$ and $\Phi=D F$.

7.3. The Generalized Kirchhoff-Sobolev Parametrix. From Propositions 43 and 44, we see that in order to control the higher-order energy quantities $\mathcal{E}^{2}(\tau)$ and $\mathcal{E}^{3}(\tau)$, uniformly for $\tau_{0} \leq \tau<t_{1}$, we must first obtain a bound for the $L^{\infty}$ norms of $R$ and the matter field: $D^{2} \phi$ in the E-S case, and $D F$ in the E-M case. As mentioned before, we will accomplish this by using the wave equations (118), (119), and (120), along with the generalized Kirchhoff-Sobolev parametrix of [22].

In the vacuum analogue of [17, one needed an $L^{\infty}$-bound for $R$, which was obtained using the Kirchhoff-Sobolev parametrix of [15]. Although this parametrix also suffices in the E-S setting, it fails in the E-M case due to first-order terms present in (118) and (120). Such terms must be handled differently than allowed in [15]; this is the primary motivation behind the generalized formula of [22.

Consider the scalar wave equation in the Minkowski spacetime $\mathbb{R}^{1+3}$ :

$$
\square \phi=\psi,\left.\quad \phi\right|_{t=0}=\alpha_{0},\left.\quad \partial_{t} \phi\right|_{t=0}=\alpha_{1} .
$$

From standard theory, cf. [10, Sec. 2.4], at a point $(t, x) \in(0, \infty) \times \mathbb{R}^{3}$, we can express $\phi(t, x)$ explicitly in terms of $\psi, \alpha_{0}$, and $\alpha_{1}$. Moreover, the above can be written as an integral along the past null cone segment $N$ from the vertex $(t, x)$ to the timeslice $t=0$, along with an "initial data" integral on the set $N \cap\{t=0\}$.

The goals of both [22] and its predecessor [15] are to provide a local first-order extension of the standard formula to arbitrary curved spacetimes. In particular, the parametrices of [15, 22 enjoy the following features:

- These formulas treat tensorial wave equations in a completely covariant fashion, without first expressing them as scalar equations.

- The parametrices are supported entirely on past null cones.

- The parametrices are valid only on regular (i.e., smooth) portions of past null cones. In the setting of this paper, this means that the formulas are only applicable up to the null injectivity radius.

- The parametrices contain "error terms", expressed as integrals along regular past null cones. These are a result of the nontrivial geometry.

- The parametrices depend only on quantities defined on the past null cones, i.e., it is independent of the extensions of such quantities off the cones. 
- The parametrices can be systematically generalized to covariant wave equations on arbitrary vector bundles over an arbitrary curved spacetime. 35

The parametrix of 22] differs from that of [15] in the following respects:

- The formula of 22 directly handles systems of tensor wave equations with additional first-order terms. The first-order terms are treated by altering the transport equation associated with the parametrix.

- While the proofs in [15 make heavy use of distributions on manifolds in an informal fashion, the proofs in 22 instead remain entirely at the level of rigorous calculus operations on null cones.

- The computations in [15] generated terms not supported on the null cone, which must all be meticulously cancelled in later steps. In [22], on the other hand, the entire derivation was performed on the null cone. More specifically, the extra terms in 15 could be avoided altogether if one integrates by parts only the derivatives tangential to the null cone.

- As a consequence of the above modifications, one can slightly weaken the assumptions listed in 15 for the parametrix to be valid. 36

For more details on the preceding discussions, see [22, or [21, Ch. 5].

We now state a special case of the main result of [22, adapted to the setting of this paper. More specifically, we state the representation formula only in the case of time foliations of the null cone, while 22 also considered arbitrary foliating functions. For the general version, see [22, Thm. 7].

Theorem 46. Assume the following:

- Let $n$ be a positive integer, let $r^{(1)}, \ldots, r^{(n)}$ be nonnegative integers, and suppose for each $1 \leq m, c \leq n$, we have defined tensor fields

$$
\Phi^{(m)} \in \Gamma T^{r^{(m)}} M, \quad \Psi^{(m)} \in \Gamma T^{r^{(m)}} M, \quad P^{(m c)} \in \Gamma T^{1+r^{(m)}+r^{(c)}} M .
$$

- Suppose the $\Phi^{(m)}$ 's, $\Psi^{(m)}$ 's, and $P^{(m c)}$ 's satisfy the system

$$
\square_{g} \Phi_{I}^{(m)}+\sum_{c=1}^{n} P_{\mu I}^{(m c)} D^{\mu} \Phi_{J}^{(c)}=\Psi_{I}^{(m)}, \quad 1 \leq m \leq n
$$

of tensor wave equations, where $I$ and $J$ in (126) are collections of $r^{(m)}$ and $r^{(c)}$ spacetime indices, respectively.

- Fix $p \in M$, and suppose $\mathcal{N}^{-}(p)$ is normalized and foliated by $\left.T\right|_{p}$ and $t_{p}$.

- Let $v_{0}$ be a constant such that $0<v_{0} \leq \mathfrak{i}(p)$.

- For each $1 \leq m \leq n$, we define the extrinsic tensor fields

$$
B^{(m)} \in \Gamma \bar{T}^{r^{(m)}} \mathcal{N}^{-}(p), \quad A^{(m)}=t_{p}^{-1} B^{(m)} \in \Gamma \bar{T}^{r^{(m)}} \mathcal{N}^{-}(p),
$$

along with a tensor $J^{(m)}$ of rank $r^{(m)}$ at $p$, such that the system

$$
\bar{\nabla}_{t_{p}} B^{(m) I}=-\frac{1}{2}\left[\vartheta(\operatorname{tr} \chi)-\frac{2}{t_{p}}\right] B^{(m) I}+\frac{\vartheta}{2} \sum_{c=1}^{n} P^{(c m)} 4 J^{I} B^{(c)} J, \quad 1 \leq m \leq n
$$

of transport equations is satisfied, along with the initial conditions

$$
\left.B^{(m)}\right|_{p}=J^{(m)}, \quad 1 \leq m \leq n .
$$

\footnotetext{
${ }^{35}$ In this extended setting, one also needs a bundle metric and a compatible connection.

${ }^{36}$ In fact, this weakening of the assumptions applies to both the formulas of [15] and [22], since the results in 15 are strictly special cases of those in 22 .
} 
- Define the "error coefficients"

$$
\nu^{(c m)} \in \Gamma \bar{T}^{r^{(c)}+r^{(m)}} \mathcal{N}^{-}(p), \quad 1 \leq m, c \leq n
$$

by the formulas

$$
\begin{aligned}
& \nu_{J}^{(c m)}{ }_{J}^{I}=-\bar{\nabla}^{a} P^{(c m)}{ }_{a J}^{I}+\frac{1}{2} \bar{\nabla}_{4} P_{3 J}^{(c m)}+\zeta^{a} P^{(c m)}{ }_{a J}^{I}+\frac{1}{4}(\operatorname{tr} \underline{\chi}) P^{(c m)}{ }_{4 J}^{I} \\
& +\frac{1}{4}(\operatorname{tr} \chi) P^{(c m)}{ }_{3 J^{I}}+\frac{1}{2} \sum_{d=1}^{n} P^{(c d)}{ }_{4 J}^{K} P^{(d m)}{ }_{3 K}{ }^{I} .
\end{aligned}
$$

Then, we have the representation formula

(130) $\left.4 \pi \cdot n(p) \cdot \sum_{m=1}^{n} J^{(m) I} \Phi^{(m)}\right|_{p}=\mathfrak{F}\left(p ; v_{0}\right)+\mathfrak{E}^{1}\left(p ; v_{0}\right)+\mathfrak{E}^{2}\left(p ; v_{0}\right)+\mathfrak{I}\left(p ; v_{0}\right)$, where:

- The "fundamental solution term" $\mathfrak{F}\left(p ; v_{0}\right)$ is given by

$$
\mathfrak{F}\left(p ; v_{0}\right)=-\sum_{m=1}^{n} \int_{\mathcal{N}^{-}\left(p ; v_{0}\right)} A^{(m) I} \Psi^{(m)}{ }^{\prime}
$$

- The "principal error terms" $\mathfrak{E}^{1}\left(p ; v_{0}\right)$ are given by

$$
\begin{aligned}
\mathfrak{E}^{1}\left(p ; v_{0}\right)=- & \sum_{m=1}^{n} \int_{\mathcal{N}^{-}\left(p ; v_{0}\right)} \bar{\nabla}^{a} A^{(m) I} \bar{\nabla}_{a} \Phi^{(m)}{ }_{I} \\
& +\sum_{m=1}^{n} \int_{\mathcal{N}^{-}\left(p ; v_{0}\right)}\left(\zeta^{a}-\underline{\eta}^{a}\right) \bar{\nabla}_{a} A^{(m) I} \Phi^{(m)}{ }_{I} .
\end{aligned}
$$

- The remaining "error terms" $\mathfrak{E}^{2}\left(p ; v_{0}\right)$ are given by

$$
\begin{aligned}
\mathfrak{E}^{2}\left(p ; v_{0}\right)=\sum_{m=1}^{n} & \int_{\mathcal{N}^{-}\left(p ; v_{0}\right)} \mu \cdot A^{(m) I} \Phi^{(m)} I \\
& +\frac{1}{2} \sum_{m=1}^{n} \int_{\mathcal{N}^{-}\left(p ; v_{0}\right)} A^{(m) I} R_{43}\left[\Phi^{(m)}\right]_{I} \\
& -\sum_{m, c=1}^{n} \int_{\mathcal{N}^{-}\left(p ; v_{0}\right)} P^{(c m)}{ }_{a J} I \bar{\nabla}^{a} A^{(c) I} \Phi^{(m)} I \\
& +\sum_{m, c=1}^{n} \int_{\mathcal{N}^{-}\left(p ; v_{0}\right)} \nu^{(c m)} J^{I} \cdot A^{(c) J} \Phi^{(m)} I .
\end{aligned}
$$

- The "initial value terms" $\Im\left(p ; v_{0}\right)$ are given by

$$
\begin{aligned}
\mathfrak{I}\left(p ; v_{0}\right)=- & \frac{1}{2} \sum_{m=1}^{n} \int_{\mathcal{S}_{v_{0}}}(\operatorname{tr} \underline{\chi}) A^{(m) I} \Phi^{(m)} I-\sum_{m=1}^{n} \int_{\mathcal{S}_{v_{0}}} A^{(m) I} D_{3} \Phi^{(m)} I \\
& -\frac{1}{2} \sum_{m, c=1}^{n} \int_{\mathcal{S}_{v_{0}}} P^{(c m)}{ }_{3 J}^{I} A^{(c) J} \Phi^{(m)}{ }_{I} .
\end{aligned}
$$

Here, we have indexed with respect to arbitrary null frames $L, \underline{L}, e_{1}, e_{2}$ adapted to the $t_{p}$-foliation. The capital letters $I, J$ refer to collections of extrinsic indices. The symbols $\chi, \underline{\chi}, \zeta$, and $\underline{\eta}$ refer to the Ricci coefficients of $\mathcal{N}^{-}(p)$. 
Remark. It is easy to see that Theorem 46 is a special case of [22, Thm. 7]. The function $t_{p}$ is clearly a foliating function in the sense of [22, Sec. 2.2], and the associated null lapse $\vartheta$ has initial value $n(p)$ at $p$. Moreover, both $\mathcal{N}^{-}(p)$ and $\mathcal{N}^{-}\left(p ; v_{0}\right)$, as given in Theorem 46, are regular portions of the full null cone $N^{-}(p)$.

Remark. Although the representation formula was stated in (130)-(134) in index notation, this was done only as a matter of convenience. It is easy to see that these expressions can in fact be described invariantly.

Remark. In particular, we can use (130) to examine the value of any $\left.\Phi^{(m)}\right|_{p}$ individually by setting $J^{(c)}=0$ for all $c \neq m$.

Lastly, we note that the $A^{(m)}$ 's in Theorem 46 satisfy the transport equations

$$
\bar{\nabla}_{L} A^{(m) I}=-\frac{1}{2}(\operatorname{tr} \chi) A^{(m) I}+\frac{1}{2} \sum_{c=1}^{n} P^{(c m)}{ }^{I}{ }^{I} A^{(c)} J, \quad 1 \leq m \leq n .
$$

7.4. Applying the Parametrix. We now describe how Theorem 46 is applied. Let $0<\delta_{0} \leq 1$ be sufficiently small such that Theorem 33 is satisfied. 37 Fix a point $p \in M_{+}$, fix another constant $0<\delta \leq \min \left(\delta_{0}, t(p)-\tau_{0}\right)$, and define $\mathcal{N}=\mathcal{N}^{-}(p ; \delta)$, normalized and foliated by $\left.T\right|_{p}$ and $t_{p}$, as usual.

In the E-S case, we wish to find $L^{\infty}$-bounds for both $R$ and $D^{2} \phi$ at $p$. As a result, appropriating the notations of Theorem 46 we set $n=2, \Phi^{(1)}=R$, and $\Phi^{(2)}=D^{2} \phi$. For the corresponding system of wave equations comprising (126), we take (118) and (119). Moreover, the right-hand sides of (118) and (119) determine the $\Psi^{(m)}$ 's and $P^{(\mathrm{cm})}$ 's. In particular, in the E-S case, the wave equations contain no first-order terms, that is, the $P^{(\mathrm{cm})}$ 's vanish entirely. 38

The E-M case is analogous, except that we must obtain $L^{\infty}$-bounds for $R$ and $D F$. We apply Theorem 46 by setting $n=2, \Phi^{(1)}=R$, and $\Phi^{(2)}=D F$, and we adopt (118) and (120) as the system of wave equations. In contrast to the E-S setting, the first-order coefficients $P^{(\mathrm{cm})}$ are no longer trivial. In particular, we see from the right-hand sides of $\left(\underline{118)}\right.$ and $(120)$ that $P^{(11)}$ and $P^{(22)}$ vanish, while $P^{(12)}$ and $P^{(21)}$ are sums of terms, each of which can be expressed as tensor products and contractions of $F$ with instances of $g$. More specifically, the $P^{(12)}$ and $P^{(21)}$ terms arise from the first, second, and seventh terms of (118) in the E-M expansion of $\tilde{d}^{2}$ Ric, and from the first term of (120). The remaining terms on the right-hand sides of (118) and (120) comprise the $\Psi^{(m)}$ 's.

Now that we have determined the $P^{(\mathrm{cm})}$ 's, we can determine their a priori bounds on $\mathcal{N}$. By the breakdown criterion (40), we have the uniform bounds

$$
\left\|P^{(c m)}\right\|_{L^{\infty}(\mathcal{N})} \lesssim 1, \quad 1 \leq m, c \leq 2
$$

in both the E-S and E-M settings. Furthermore, it follows from Propositions 24 and 25 that in both the E-S and E-M cases,

$$
\left\|\bar{\nabla} P^{(c m)}\right\|_{L^{2}(\mathcal{N})}+\left\|\bar{\nabla}_{t_{p}} P^{(c m)}\right\|_{L^{2}(\mathcal{N})} \lesssim 1, \quad 1 \leq m, c \leq 2 .
$$

Of course, in the E-S case, the bounds (136) and (137) hold trivially.

\footnotetext{
${ }^{37}$ More explicitly, we assume that given any $q \in M_{+}$, then $\mathfrak{i}(q) \geq \delta_{1}=\min \left(\delta_{0}, t(q)-\tau_{0}\right)$, and the estimates (109) and (110) hold on $\mathcal{N}_{q}=\mathcal{N}^{-}\left(q ; \delta_{1}\right)$.

${ }^{38}$ Consequently, the parametrix of [15] suffices in the E-S setting.
} 
Finally, we choose arbitrary tensors $J^{(1)}$ and $J^{(2)}$ at $p$, of the same ranks as $\Phi^{(1)}$ and $\Phi^{(2)}$, respectively. For convenience, we adopt the abbreviation

$$
|J|=\left|J^{(1)}\right|+\left|J^{(2)}\right| .
$$

Applying Theorem 46, we obtain the expansion (130) for the quantity

$$
4 \pi \cdot n(p) \cdot\left(\left.J^{(1) I} \Phi^{(1)}\right|_{p}+\left.J^{(2) I} \Phi^{(2)}\right|_{p}\right) .
$$

Each term of this expansion is either an integral over $\mathcal{N}$ (i.e., the terms $\mathfrak{F}(p ; \delta)$, $\mathfrak{E}^{1}(p ; \delta)$, and $\left.\mathfrak{E}^{2}(p ; \delta)\right)$ or an "initial value" integral over the $t_{p}$-level set $\mathcal{S}_{\delta}$ (i.e., the terms $\mathfrak{I}(p ; \delta))$. Thus, in order to bound $\Phi^{(1)}$ and $\Phi^{(2)}$ at $p$, as desired, we must bound each of the integral terms mentioned above.

Before we can accomplish this, though, we must first control the fields $B^{(m)}$ and $A^{(m)}$ associated with the system of transport equations (127), (128), and (135). This is the content of the subsequent proposition, which is the E-S and E-M analogue of the estimates [17, Prop. 7.1, Prop. 7.3] in the vacuum case.

Proposition 47. In both the $E$-S and E-M settings, we have

$$
\sum_{m=1}^{2}\left\|B^{(m)}\right\|_{L^{\infty}(\mathcal{N})} \lesssim|J|, \quad \sum_{m=1}^{2}\left\|\bar{\nabla} A^{(m)}\right\|_{L^{2}(\mathcal{N})} \lesssim|J|,
$$

as long as $\delta_{0}$ is sufficiently small with respect to the fundamental constants.

Proof. By standard calculus computations analogous to those of Proposition 4,

$$
\begin{aligned}
\bar{\nabla}_{t_{p}}\left|B^{(m)}\right|^{2} & \lesssim\left|B^{(m)}\right|\left|\bar{\nabla}_{t_{p}} B^{(m)}\right|+\left|\bar{\nabla}_{t_{p}} h\right|\left|B^{(m)}\right|^{2} \\
& \lesssim\left|B^{(m)}\right|\left[|I|\left|B^{(m)}\right|+\sum_{c=1}^{2}\left|P^{(c m)}\right|\left|B^{(c)}\right|\right]+\left|B^{(m)}\right|^{2},
\end{aligned}
$$

where $I=\vartheta(\operatorname{tr} \chi)-2 t_{p}^{-1}$ and $h$ is the induced Riemannian metric on $M$. Here, we have applied (39) and (127). Summing over $m$ and applying (110) and (136), then

$$
\bar{\nabla}_{t_{p}}\left(\sum_{m=1}^{2}\left|B^{(m)}\right|^{2}\right) \lesssim\left(1+|I|+\sum_{c, m=1}^{2}\left|P^{(c m)}\right|\right) \sum_{m=1}^{2}\left|B^{(m)}\right|^{2} \lesssim \sum_{m=1}^{2}\left|B^{(m)}\right|^{2} .
$$

Applying Grönwall's inequality to the above while taking into account the initial conditions (128), we obtain the first desired estimate.

Next, we define

$$
U^{(m)}=t_{p}^{2} \cdot \bar{\nabla} A^{(m)} \in \Gamma \underline{T}^{1} \bar{T}^{r^{(m)}} \mathcal{N}^{-}(p), \quad|V|=\max \left(\left|\bar{\nabla} A^{(1)}\right|,\left|\bar{\nabla} A^{(2)}\right|\right) .
$$

Differentating the transport equations (135) and commuting derivatives (see the commutation formula [21, Prop. 3.12]), we obtain the transport equations

$$
\begin{aligned}
\bar{\nabla}_{t_{p}} U^{(m)}{ }_{a K}=- & \cdot U^{(m)}{ }_{a K}-\vartheta \hat{\chi}_{a}{ }^{b} U^{(m)}{ }_{b K}+\frac{1}{2} \vartheta \sum_{c=1}^{2} P^{(c m)}{ }_{4}{ }^{C}{ }_{K} U^{(c)}{ }_{a C} \\
& -\frac{1}{2} t_{p} \not_{a} I \cdot B^{(m)} K+t_{p} \vartheta \sum_{i=1}^{r^{(m)}} R_{\gamma_{i}}{ }^{\mu}{ }_{4 a} B^{(m)} K_{\mu}^{i} \\
& +\frac{1}{2} t_{p} \sum_{c=1}^{2}\left(\not_{a} \vartheta P^{(c m)}{ }_{4}{ }^{C}{ }_{K}+\vartheta \bar{\nabla}_{a} P^{(c m)}{ }_{4}{ }^{C}{ }_{K}\right) B^{(c)}{ }_{C},
\end{aligned}
$$


where $K=\left(\gamma_{1}, \ldots, \gamma_{r(m)}\right)$ and $C$ denote collections of $r^{(m)}$ and $r^{(c)}$ extrinsic indices, respectively, and where $K_{\mu}^{i}$ denotes $K$ except with the $i$-th index replaced by $\mu$. In addition, we index both the horizontal and the extrinsic components of the $U^{(m)}$ 's using local Fermi transported null frames. 39 If we also (locally) define

$$
\mathcal{C}=\left|\bar{D}_{L} e_{1}\right|+\left|\bar{D}_{L} e_{2}\right|+\left|\bar{D}_{L} L\right|+\left|\bar{D}_{L} \underline{L}\right|,
$$

then $\mathcal{C} \lesssim|\underline{\eta}| \lesssim 1$ by Proposition 37 , and

$$
\left|\overline{\mathbb{X}}_{t_{p}}\left(U^{(m)}{ }_{a K}\right)\right| \lesssim\left|\overline{\mathbb{W}}_{t_{p}} U^{(m)}\right|+\mathcal{C}\left|U^{(m)}\right| \lesssim\left|\overline{\mathbb{W}}_{t_{p}} U^{(m)}\right|+\left|U^{(m)}\right|,
$$

where the left-hand side denotes $\bar{\nabla}_{t_{p}}$ applied to the scalar quantity $U^{(m)}{ }_{a K}$.

With consideration of the coefficients present in (138), we define

$$
\begin{aligned}
& \mathcal{Q}_{1}=|I|+|\hat{\chi}|+\sum_{c, m=1}^{2}\left|P^{(c m)}\right|, \\
& \mathcal{Q}_{2}=|\not \nabla I|+|\not \nabla \vartheta| \sum_{c, m=1}^{2}\left|P^{(c m)}\right|-Q_{m}[R](T, L)+\sum_{c, m=1}^{2}\left|\bar{\nabla} P^{(c m)}\right| .
\end{aligned}
$$

In addition, by Proposition 24, (109), Proposition 37, (136), and (137), we obtain

$$
\left\|\mathcal{Q}_{1}\right\|_{L_{\omega}^{\infty} L_{t_{p}}^{2}(\mathcal{N})} \lesssim 1, \quad\left\|\mathcal{Q}_{2}\right\|_{L^{2}(\mathcal{N})} \lesssim 1
$$

We now integrate (138) along each null generator of $\mathcal{N}$. By also noting Corollary 26 and (139), then we obtain the inequality

$$
\begin{aligned}
v^{2}|V|_{(v, \omega)} & \lesssim \int_{0}^{v} w^{2}\left(1+\mathcal{Q}_{1}\right)|V|_{(w, \omega)} d w+\left.|J| \int_{0}^{v} w \mathcal{Q}_{2}\right|_{(w, \omega)} d w \\
& \lesssim\left(\left.\int_{0}^{v} w^{4}|V|^{2}\right|_{(w, \omega)} d w\right)^{\frac{1}{2}}+\left.|J| \int_{0}^{v} w \mathcal{Q}_{2}\right|_{(w, \omega)} d w
\end{aligned}
$$

for each $0<v<\delta$ and $\omega \in \mathbb{S}^{2}$, where we also applied the preceding bound $\left|B^{(m)}\right| \lesssim|J|$ and the estimate (140) for $\mathcal{Q}_{1}$. Dividing both sides of the above by $v$, taking $L_{t}^{2} L_{\omega}^{2}$-norms of the resulting inequality (cf. (107)), and then recalling the norm comparisons of Proposition 34, we obtain

$$
\begin{aligned}
\||V|\|_{L^{2}(\mathcal{N})}^{2} \lesssim & \int_{0}^{\delta} \int_{\mathbb{S}^{2}}\left(\left.\int_{0}^{v} w^{2}|V|^{2}\right|_{(w, \omega)} d w\right) d \omega d v \\
& +|J|^{2} \int_{\mathbb{S}^{2}} \int_{0}^{\delta}\left(\left.v^{-1} \int_{0}^{v} w \mathcal{Q}_{2}\right|_{(w, \omega)} d w\right)^{2} d v d \omega \\
\lesssim & \delta_{0}\||V|\|_{L^{2}(\mathcal{N})}^{2}+\left.|J|^{2} \int_{\mathbb{S}^{2}} \int_{0}^{\delta} v^{2} \mathcal{Q}_{2}\right|_{(v, \omega)} d v d \omega \\
\lesssim & \delta_{0}\||V|\|_{L^{2}(\mathcal{N})}^{2}+|J|^{2} .
\end{aligned}
$$

where we applied Proposition 34 to the $V$-term, and where we applied Hardy's inequality and (140) to the $\mathcal{Q}_{2}$-term. Shrinking $\delta_{0}$ if necessary (still depending only on the fundamental constants), then $\||V|\|_{L^{2}(\mathcal{N})} \lesssim|J|$, completing the proof.

\footnotetext{
${ }^{39} \mathrm{By}$ this, we mean null frames $L, \underline{L}, e_{1}, e_{2}$ satisfying the conditions $\nabla_{L} e_{a} \equiv 0$.
} 
7.5. The Uniform Bounds. We described in Section 7.4 how Theorem 46 is applied to the breakdown problem. With the preliminary work in place, we must now bound each of the resulting terms $\mathfrak{F}(p, \delta), \mathfrak{E}^{1}(p, \delta), \mathfrak{E}^{2}(p, \delta)$, and $\mathfrak{I}(p, \delta)$. For convenience, let $\Theta$ denote $D^{2} \phi$ in the E-S case and $D F$ in the E-M case. In addition, we define for every $\tau_{0} \leq \tau<t_{1}$ the uniform norm

$$
\mathcal{S}(\tau)=\|R\|_{L^{\infty}\left(\Sigma_{\tau}\right)}+\|\Theta\|_{L^{\infty}\left(\Sigma_{\tau}\right)}
$$

We start with the principal "fundamental solution" term $\mathfrak{F}(p ; \delta)$, given by (131). First of all, we decompose the integrands $A^{(m) I} \Psi^{(m)} I$ using local null frames. Since $t_{p} A^{(m)}=B^{(m)}$ is uniformly bounded by Proposition 47 , then the main challenge is to bound the integral of every component $t_{p}^{-1} \Psi^{(m)}{ }_{I}$ along $\mathcal{N}$. The primary observation is the following, which is also the cornerstone of [9, 17. From (118)-(120), we see that each term of any component $\Psi^{(m)} I$ is of the schematic form $W^{2} \cdot Q$ or $Q^{2}$, where $\|W\|_{L^{\infty}(\mathcal{N})} \lesssim 1$, and where $Q$ denotes a tensor field of the form $R$ or $\Theta$. 40

The first form $W^{2} \cdot Q$ can be sufficiently controlled as follows:

$$
\int_{\mathcal{N}}\left|A^{(m)}\right||W|^{2}|Q| \lesssim|J| \int_{\mathcal{N}} t_{p}^{-1}|Q| \lesssim \delta^{2}|J| \sup _{t(p)-\delta \leq \tau \leq t(p)} \mathcal{S}(\tau)
$$

Note in particular that we have applied Proposition 47 to control the $A^{(m)}$ 's. The terms of type $Q^{2}$ require a more detailed null frame decomposition. The main observation is the following: for each component of every $Q^{2}$-term, one of the $Q$ factors is bounded by the flux density $\left[\rho^{1}(p, t(p)-\delta)\right]^{1 / 2}$, which can be controlled using Proposition 24. We shall further elaborate on this point later in this section.

Assuming for now the above observation, then

$$
\int_{\mathcal{N}} A^{(m)} \cdot Q^{2} \lesssim|J|\left[\mathcal{F}^{1}(p ; t(p)-\delta)\right]^{\frac{1}{2}}\left(\int_{\mathcal{N}} t_{p}^{-2}|Q|^{2}\right)^{\frac{1}{2}} \lesssim \delta^{\frac{1}{2}}|J| \sup _{t(p)-\delta \leq \tau \leq t(p)} \mathcal{S}(\tau)
$$

by Proposition 24. Combining the above, we obtain the estimate

$$
\mathfrak{F}(p ; \delta) \lesssim \delta^{\frac{1}{2}}|J| \sup _{t(p)-\delta \leq \tau \leq t(p)} \mathcal{S}(\tau) \lesssim \delta_{0}^{\frac{1}{2}}|J| \sup _{t(p)-\delta \leq \tau \leq t(p)} \mathcal{S}(\tau) .
$$

Remark. Recall that $\delta_{0}>0$ is the constant, depending only on the fundamental constants, for which Theorem 33 holds, while $0<\delta \leq \min \left(\delta_{0}, t(p)-\tau_{0}\right)$.

We now discuss handling the $Q^{2}$-terms in further detail. The process is analogous to the E-V case of [17, except we must consider several additional terms involving the matter field. Recall that the exact null frame components controlled by the flux density $\rho^{1}(p, t(p)-\delta)$ were determined in detail in Proposition 25 and in the subsequent discussions. More specifically, the only components of $R$ and $\Theta$ that are not controlled are those of the forms $R_{3 a 3 b}, D_{33} \phi$ (in the E-S case), and $D_{3} F_{3 a}$ (in the E-M case). As a result, we must determine that for every $Q^{2}$-term in the $\Psi^{(m)}$ 's, at least one of the $Q$ 's is not an "invalid" component.

We give a few examples here demonstrating the reasoning described above:

- Consider the term $I_{\alpha \beta \gamma \delta}=\mathfrak{A}_{\alpha \beta}\left[D_{\alpha \gamma} \phi D_{\beta \delta} \phi\right]$ in the expansion of (118) in the E-S case. By the above characterizations, the only invalid component, for which neither factor is controlled, is $D_{33} \phi D_{33} \phi$. To obtain this component,

\footnotetext{
${ }^{40}$ Recall that in the E-M case, the first-order terms (corresponding to the $P^{(\mathrm{cm})}$ 's) are omitted from the $\Psi^{(m)}$ 's. In fact, the reason that the original parametrix of [15] fails here is precisely because these first-order terms are not of the above forms $W^{2} \cdot Q$ or $Q^{2}$.
} 
we require $\alpha=\beta=\gamma=\delta=3$; but, by the antisymmetry between $\alpha$ and $\beta$, the component $I_{3333}$ vanishes. Consequently, any component of $I_{\alpha \beta \gamma \delta}$ can be bounded by $-Q_{w}[D \phi](T, L) \cdot\left|D^{2} \phi\right| \lesssim\left[\rho^{1}(p, t(p)-\delta)\right]^{1 / 2} \cdot\left|D^{2} \phi\right|$.

- The term $I_{\alpha \beta \gamma \delta}=D^{\mu} F_{\alpha \beta} D_{\mu} F_{\gamma \delta}$ in the E-M expansion of (118) can be handled similarly. Because of the contraction involving $\mu$ and the properties of null frames, for each term of the summation, one of the $\mu$ 's must not be "3". As a result, one $D F$ factor can always be bounded by $-Q_{w}[F](T, L)$.

- The terms $R_{\alpha}{ }^{\lambda}{ }^{\mu} R_{\mu \lambda \gamma \delta}$ and $\mathfrak{A}_{\alpha \beta}\left[R_{\alpha}{ }^{\mu}{ }_{\gamma}{ }^{\lambda} R_{\beta \mu \delta \lambda}\right]$ in (118) correspond with the $R^{2}$-terms present in the E-V setting in [17. They can be handled using signature considerations, as in [17, Sec. 5.4], or directly using the same reasoning as for the previous two examples. More explicitly, we see that at least one of the $R$ 's is controlled by $-Q_{m}[R](T, L)$.

- For the term $R_{\alpha}{ }^{\mu}{ }_{\beta}{ }^{\lambda} D_{\mu \lambda} \phi$ in (119), due to the contractions, for each term in this summation, the " $D^{2} \phi$ " factor can be bounded by $-Q_{w}[D \phi](T, L)$ unless $\mu=\lambda=3$ in $D^{2} \phi$. If this is true, however, then $\mu=\lambda=4$ in the " $R$ " factor, which can hence be bounded by $-Q_{m}[R](T, L)$.

The remaining $Q^{2}$-terms in (118)-(120) can be handled using similar reasoning.

The next task is to bound the "error terms" $\mathfrak{E}^{1}(p ; \delta)$ and $\mathfrak{E}^{2}(p ; \delta)$, defined in (132) and (133). First, by Propositions 45] and 47, we have

$$
\begin{aligned}
\int_{\mathcal{N}}\left|\bar{\not} A^{(m)}\right|\left|\bar{\nabla} \Phi^{(m)}\right| & \lesssim\left\|\bar{\not} A^{(m)}\right\|_{L^{2}(\mathcal{N})}|| \bar{\not} \Phi^{(m)} \|_{L^{2}(\mathcal{N})} \\
& \lesssim|J|\left[\mathcal{E}^{2}(t(p)-\delta)^{\frac{1}{2}}+\delta^{\frac{1}{2}} \sup _{t(p)-\delta \leq \tau \leq t(p)} \mathcal{S}(\tau)\right] .
\end{aligned}
$$

Similarly, by (109) and Proposition 47.

$$
\begin{aligned}
\int_{\mathcal{N}}|\zeta-\underline{\eta}|\left|\bar{\nabla} A^{(m)}\right|\left|\Phi^{(m)}\right| & \lesssim\|\zeta-\underline{\eta}\|_{L^{2}(\mathcal{N})}\left\|\bar{\nabla} A^{(m)}\right\|_{L^{2}(\mathcal{N})}\left\|\Phi^{(m)}\right\|_{L^{\infty}(\mathcal{N})} \\
& \lesssim \delta|J| \sup _{t(p)-\delta \leq \tau \leq t(p)} \mathcal{S}(\tau) .
\end{aligned}
$$

Therefore, we obtain the bound

$$
\mathfrak{E}^{1}(p ; \delta) \lesssim\left[\mathcal{E}^{2}(t(p)-\delta)\right]^{\frac{1}{2}}+\delta_{0}^{\frac{1}{2}}|J| \sup _{t(p)-\delta \leq \tau \leq t(p)} \mathcal{S}(\tau) .
$$

Next, for the first term of (133),

$$
\begin{aligned}
\int_{\mathcal{N}}|\mu|\left|A^{(m)}\right|\left|\Phi^{(m)}\right| & \lesssim\|\mu\|_{L^{2}(\mathcal{N})}\left\|A^{(m)}\right\|_{L^{2}(\mathcal{N})}\left\|\Phi^{(m)}\right\|_{L^{\infty}(\mathcal{N})} \\
& \lesssim \delta^{\frac{1}{2}}|J| \sup _{t(p)-\delta \leq \tau \leq t(p)} \mathcal{S}(\tau)
\end{aligned}
$$

where we appealed to (110) to bound $\mu$. For the second term, by expanding $R_{43}\left[\Phi^{(m)}\right]$ using null frames, we see that each term of this expansion is bounded by $-Q_{m}[R](T, L) \cdot\left|\Phi^{(m)}\right|$. As a result,

$$
\int_{\mathcal{N}}\left|A^{(m)}\right|\left|R_{43}\left[\Phi^{(m)}\right]\right| \lesssim \delta^{\frac{1}{2}}|J| \sup _{t(p)-\delta \leq \tau \leq t(p)} \mathcal{S}(\tau) .
$$

By (136) and Proposition 47, we can bound the third term:

$$
\int_{\mathcal{N}}\left|P^{(c m)}\right|\left|\overline{\mathbb{X}} A^{(c)}\right|\left|\Phi^{(m)}\right| \lesssim\left\|P^{(c m)}\right\|_{L^{2}(\mathcal{N})}\left\|\overline{\mathbb{X}} A^{(c)}\right\|_{L^{2}(\mathcal{N})}\left\|\Phi^{(m)}\right\|_{L^{\infty}(\mathcal{N})}
$$




$$
\lesssim \delta^{\frac{3}{2}}|J| \sup _{t(p)-\delta \leq \tau \leq t(p)} \mathcal{S}(\tau)
$$

For the last term, we will need the following simple estimate:

Lemma 48. The coefficients $\nu^{(\mathrm{cm})}$, as defined in (129), satisfy

$$
\left\|\nu^{(c m)}\right\|_{L^{2}(\mathcal{N})} \lesssim 1, \quad c, m \in\{1,2\}
$$

Proof. We bound each term on the right-hand side of (129). The first two terms are trivially bounded using (137); by Theorem 33, Proposition 37, and (136), the third, fourth, and fifth terms are bounded by $\delta_{0}^{1 / 2}$. The final term on the right-hand side of (129) is trivially bounded using (136).

Finally, applying Lemma 48, then

$$
\begin{aligned}
\int_{\mathcal{N}}\left|\nu^{(c m)}\right|\left|A^{(c)}\right|\left|\Phi^{(m)}\right| & \lesssim\left\|\nu^{(c m)}\right\|_{L^{2}(\mathcal{N})}\left\|A^{(c)}\right\|_{L^{2}(\mathcal{N})}\left\|\Phi^{(m)}\right\|_{L^{\infty}(\mathcal{N})} \\
& \lesssim \delta^{\frac{1}{2}}|J| \sup _{t(p)-\delta \leq \tau \leq t(p)} \mathcal{S}(\tau)
\end{aligned}
$$

As a result,

$$
\mathfrak{E}^{2}(p ; \delta) \lesssim \delta_{0}^{\frac{1}{2}}|J| \sup _{t(p)-\delta \leq \tau \leq t(p)} \mathcal{S}(\tau) .
$$

It remains only to bound the initial value terms $\mathfrak{I}(p ; \delta)$ in (134). The first term on the right-hand side of (134) can be handled using Proposition 34 and 37

$$
\int_{\mathcal{S}_{\delta}}(\operatorname{tr} \underline{\chi})\left|A^{(m)}\right|\left|\Phi^{(m)}\right| \lesssim \delta^{-2}|J| \int_{\mathcal{S}_{\delta}}\left|\Phi^{(m)}\right| \lesssim|J|\left\|\Phi^{(m)}\right\|_{L^{\infty}\left(\Sigma_{t(p)-\delta}\right)} .
$$

Applying (72) and Proposition 21, then the above is bounded by

$$
|J|\left[1+\mathcal{E}^{2}(t(p)-\delta)+\mathcal{E}^{3}(t(p)-\delta)\right]^{\frac{1}{2}} .
$$

The third term of (134) can be bounded similarly:

$$
\int_{\mathcal{S}_{\delta}}\left|P^{(c m)}\right|\left|A^{(c)}\right|\left|\Phi^{(m)}\right| \lesssim \delta|J|\left[1+\mathcal{E}^{2}(t(p)-\delta)+\mathcal{E}^{3}(t(p)-\delta)\right]^{\frac{1}{2}} .
$$

Lastly, for the remaining term of (134), we will need the following trace estimate:

Lemma 49. If $Z \in \Gamma \overline{\mathcal{T}} \mathcal{N}^{-}(p)$ and $W \in \Gamma \mathcal{T} M$ are of the same rank, then

$$
\left|\int_{\mathcal{S}_{\delta}} Z^{I} W_{I}\right| \lesssim \delta^{\frac{3}{2}}\|Z\|_{L^{\infty}\left(\mathcal{S}_{\delta}\right)}\left(\|\bar{\nabla} W\|_{L^{2}\left(\Sigma_{t(p)-\delta}\right)}+\|W\|_{L^{2}\left(\Sigma_{t(p)-\delta}\right)}\right) .
$$

Proof. See [21, Prop. 7.3]. The proof involves constructing a radial foliation of $\Sigma_{t(p)-\delta}$ using the level sets of a one-parameter family of null cones. This relies heavily on Theorem 33, in particular the null injectivity radius bounds.

We now apply Lemma 49 with $Z=\underline{L} \otimes A^{(m)}$ and $W=D \Phi^{(m)}$. Since $Z$, as defined above, satisfies $\|Z\|_{L^{\infty}\left(\mathcal{S}_{\delta}\right)} \lesssim \delta^{-1}|J|$, then

$$
\begin{aligned}
\int_{\mathcal{S}_{\delta}} \underline{L}^{\alpha} A^{(m) I} D_{\alpha} \Phi_{I}^{(m)} & \lesssim \delta^{\frac{1}{2}}|J|\left(\left\|D^{2} \Phi^{(m)}\right\|_{L^{2}\left(\Sigma_{t(p)-\delta}\right)}+\left\|D \Phi^{(m)}\right\|_{L^{2}\left(\Sigma_{t(p)-\delta}\right)}\right) \\
& \lesssim \delta^{\frac{1}{2}}|J|\left[\mathcal{E}^{2}(t(p)-\delta)+\mathcal{E}^{3}(t(p)-\delta)\right]^{\frac{1}{2}} .
\end{aligned}
$$


As a result, we obtain

$$
\Im(p ; \delta) \lesssim|J|\left[1+\mathcal{E}^{2}(t(p)-\delta)+\mathcal{E}^{3}(t(p)-\delta)\right]^{\frac{1}{2}} .
$$

Combining (130) and (141)-(144), we obtain

$$
\begin{aligned}
\left|\sum_{1 \leq a \leq 2} J^{(a) I} \Phi^{(a)} I\right|_{p}\left|\lesssim \delta_{0}^{\frac{1}{2}}\right| J \mid \sup _{t(p)-\delta \leq \tau \leq t(p)} \mathcal{S}(\tau) & \\
& +|J|\left[1+\mathcal{E}^{2}(t(p)-\delta)+\mathcal{E}^{3}(t(p)-\delta)\right]^{\frac{1}{2}} .
\end{aligned}
$$

If we consider all possible values of $J^{(1)}$ and $J^{(2)}$, then we have

$$
\left|R\left\|_{p}+\mid \Theta\right\|_{p} \lesssim \delta_{0}^{\frac{1}{2}} \sup _{t(p)-\delta \leq \tau \leq t(p)} \mathcal{S}(\tau)+\left[1+\mathcal{E}^{2}(t(p)-\delta)+\mathcal{E}^{3}(t(p)-\delta)\right]^{\frac{1}{2}} .\right.
$$

Finally, if we fix $\tau$ such that $\tau_{0}<\tau<t_{1}$ and $\tau-\delta \geq \tau_{0}$, and we apply (145) to every $p \in M_{+}$satisfying $\tau-\delta \leq t(p) \leq \tau$, then we obtain

$$
\sup _{\tau-\delta \leq \tau^{\prime} \leq \tau} \mathcal{S}\left(\tau^{\prime}\right) \lesssim \delta_{0}^{\frac{1}{2}} \sup _{\tau-\delta \leq \tau^{\prime} \leq \tau} \mathcal{S}\left(\tau^{\prime}\right)+\left[1+\mathcal{E}^{2}(\tau-\delta)+\mathcal{E}^{3}(\tau-\delta)\right]^{\frac{1}{2}} .
$$

By taking $\delta_{0}$ sufficiently small, we have proven the following:

Proposition 50. Fix $\tau_{0}<\tau<t_{1}$. Suppose $\delta_{0}>0$ is sufficiently small, depending only on the fundamental constants, and let $0<\delta \leq \delta_{0}$ such that $\tau-\delta \geq \tau_{0}$. Then,

$$
\sup _{\tau-\delta \leq \tau^{\prime} \leq \tau} \mathcal{S}\left(\tau^{\prime}\right)^{2} \lesssim 1+\mathcal{E}^{2}(\tau-\delta)+\mathcal{E}^{3}(\tau-\delta) .
$$

7.6. Completion of the Proof. With the uniform bounds of Proposition [50 in place, we can now embark on the final stretch of the proof of the main theorem. We begin by applying Proposition 50 to the higher-order energy estimates of Propositions 43 and 44 This yields higher-order energy estimates.

Proposition 51. For every $\tau_{0} \leq \tau<t_{1}$, we have

$$
\mathcal{E}^{2}(\tau)+\mathcal{E}^{3}(\tau) \lesssim 1
$$

Proof. Let $\tau_{0}<\tau<t_{1}$ and $0<\delta \leq \delta_{0}$ such that $\tau-\delta \geq \tau_{0}$, where $\delta_{0}$ was determined in Proposition 50. Applying Proposition 50 to Proposition 43 yields

$$
\begin{aligned}
\mathcal{E}^{2}(\tau) & \lesssim \mathcal{E}^{2}(\tau-\delta)+\delta\left[1+\mathcal{E}^{2}(\tau-\delta)+\mathcal{E}^{3}(\tau-\delta)\right] \\
& \lesssim 1+\mathcal{E}^{2}(\tau-\delta)+\mathcal{E}^{3}(\tau-\delta) .
\end{aligned}
$$

Combining the above with Propositions 44 and [50, then

$$
\begin{aligned}
\mathcal{E}^{3}(\tau) & \lesssim \mathcal{E}^{3}(\tau-\delta)+\delta\left[1+\mathcal{E}^{2}(\tau-\delta)+\mathcal{E}^{3}(\tau-\delta)\right]^{2} \\
& \lesssim 1+\left[\mathcal{E}^{2}(\tau-\delta)+\mathcal{E}^{3}(\tau-\delta)\right]^{2} .
\end{aligned}
$$

As a result, we have derived

$$
\mathcal{E}^{2}(\tau)+\mathcal{E}^{3}(\tau) \lesssim 1+\left[\mathcal{E}^{2}(\tau-\delta)+\mathcal{E}^{3}(\tau-\delta)\right]^{2} .
$$

We can now iterate (146). First, we let $\delta^{\prime}=\min \left(\delta_{0}, t_{1}-\tau_{0}\right)$, and we apply (146) with $0<\delta<\delta^{\prime}$ and $\tau \leq \tau_{0}+\delta^{\prime}$ to obtain

$$
\sup _{\tau_{0} \leq \tau^{\prime}<\tau_{0}+\delta^{\prime}}\left[\mathcal{E}^{2}\left(\tau^{\prime}\right)+\mathcal{E}^{3}\left(\tau^{\prime}\right)\right] \lesssim 1
$$


If $\delta^{\prime}=t_{1}-\tau_{0}$ (i.e., $t_{1}-\tau_{0} \leq \delta_{0}$ ), then the proof is complete. On the other hand, if $\delta^{\prime}=\delta_{0}<t_{1}-\tau_{0}$, then (146) also yields

$$
\mathcal{E}^{2}\left(\tau_{0}+\delta_{0}\right)+\mathcal{E}^{3}\left(\tau_{0}+\delta_{0}\right) \lesssim 1
$$

We can now repeat the above procedure, but with $\tau_{0}$ replaced by $\tau_{0}+\delta_{0}$. By this process, we can reach the breakdown time $t_{1}$ within a finite number of iterations. This implies the desired result and completes the proof.

Now that the higher-order spacetime quantities are controlled, we must do the same for the corresponding horizontal quantities. Similar to the a priori estimates, we accomplish this by relating the corresponding spacetime and horizontal quantities to each other and then applying elliptic estimates. The process is analogous to that of Sections [5.6 and 5.7, except the computations are somewhat more involved. Consequently, we only list the final results and omit the proofs.

Proposition 52. For any $\tau_{0} \leq \tau<t_{1}$, the following inequalities hold:

$$
\mathfrak{R}(\tau)+\mathfrak{K}(\tau)+\mathfrak{f}(\tau) \lesssim 1
$$

Proof. This is a consequence of [21, Lemma 9.4] and Proposition 51]

Remark. The quantities $\mathfrak{R}(\tau), \mathfrak{K}(\tau)$, and $\mathfrak{f}(\tau)$ were defined in (44)-(46).

We have now successfully controlled the required horizontal energy quantities. From Section 3.4, it remains only to control the diameters and the injectivity radii of the $\Sigma_{\tau}$ 's. First of all, Propositions 17 and 52 imply uniform bounds for

$$
\|\mathcal{R}\|_{L^{\infty}\left(\Sigma_{\tau}\right)}, \quad \tau_{0} \leq \tau<t_{1} .
$$

As a result, combining the above with Proposition 15, we obtain the desired uniform control for the injectivity radii of the $\Sigma_{\tau}$ 's. 41

The diameters of the $\Sigma_{\tau}$ 's can be controlled by even cruder means using Proposition 13. For example, let $p, q \in \Sigma_{\tau}$, where $\tau_{0} \leq \tau<t_{1}$. Consider the normal transports $p_{0}, q_{0}$ of $p, q$ to $\Sigma_{\tau_{0}}$, and fix a curve $\alpha_{0}$ in $\Sigma_{\tau_{0}}$ from $p_{0}$ to $q_{0}$ whose length is controlled by the diameter of $\Sigma_{\tau_{0}}$. By using the transported coordinate systems of Proposition 13 and the uniform ellipticity property of (64), then we can bound the length of the normal transport $\alpha$ of $\alpha_{0}$ to $\Sigma_{\tau}$. This bound depends only on the fundamental constants, including the diameter of $\Sigma_{\tau_{0}}$. This controls the diameters of the $\Sigma_{\tau}$ 's and finally completes the proof of Theorem 6

\section{REFERENCES}

1. M. Anderson, On long-time evolution in general relativity and geometrization of 3-manifolds, Comm. Math. Phys. 222 (2001), 533-567.

2. L. Andersson and V. Moncrief, Elliptic-hyperbolic systems and the Einstein equations, Ann. Henri Poincaré 4 (2003), 1-34.

3. J. T. Beale, T. Kato, and A. Majda, Remarks on the breakdown of smooth solutions for the 3-D Euler equations, Comm. Math. Phys. 94 (1984), 61-66.

4. J. Cheeger, Finiteness theorems for Riemannian manifolds, American J. Math 92 (1970), $61-74$.

5. J. Cheeger, M. Gromov, and M. Taylor, Finite propagation speed, kernel estimates for functions of the Laplace operator, and the geometry of complete Riemannian manifolds, J. Differential Geom. 17 (1982), no. 1, 15-53.

\footnotetext{
${ }^{41}$ More specifically, the uniform lower bound on the injectivity radii follows from uniform bounds on the curvature and on the volume radii of geodesic balls; see, e.g., 4, 5.
} 
6. Y. Choquét-Bruhat, Théorème d'existence pour certains systèmes d'équations aux dérivées partielles nonlinéaires, Acta Math. 88 (1952), 141-225.

7. D. Christodoulou and S. Klainerman, Global nonlinear stability of the Minkowski space, Princeton University Press, 1993.

8. P. Chruściel and J. Shatah, Global existence of solutions of the Yang-Mills equations on globally hyperbolic four dimensional Lorentzian manifolds, Asian J. Math 1 (1997), no. 3, 530-548.

9. D. M. Eardley and V. Moncrief, The global existence of Yang-Mills-Higgs fields in 4dimensional Minkowski space. II. Completion of proof, Comm. Math. Phys. 83 (1982), 193212 .

10. L. C. Evans, Partial differential equations, AMS, 2002.

11. F. G. Friedlander, The wave equation on a curved spacetime, Cambridge University Press, 1976.

12. S. Klainerman and I. Rodnianski, Causal geometry of Einstein-vacuum spacetimes with finite curvature flux, Inventiones Math. 159 (2005), 437-529.

13. - A geometric approach to the Littlewood-Paley theory, Geom. Funct. Anal. 16 (2006), no. $1,126-163$.

14. Sharp trace theorems for null hypersurfaces on Einstein metrics with finite curvature flux, Geom. Funct. Anal. 16 (2006), no. 3, 164-229.

15. _ A Kirchhoff-Sobolev parametrix for the wave equation and applications, Journ. of Hyperbolic Equ. 4 (2007), no. 3, 401-433.

16. - On the radius of injectivity of null hypersurfaces, J. Amer. Math. Soc. 21 (2008), no. $3,775-795$.

17.

18. B. O'Neill, Semi-Riemannian geometry with applications to relativity, Academic Press, 1983.

19. D. Parlongue, An integral breakdown criterion for Einstein vacuum equations in the case of asymptotically flat spacetimes, Preprint, 2010.

20. S. Selberg, Lecture notes. Math 632, PDE, Lecture notes on nonlinear wave equations, Spring 2001, Johns Hopkins University.

21. A. Shao, Breakdown criteria for nonvacuum Einstein equations, Ph.D. thesis, Princeton University, 2010.

22. $\ldots$ A generalized representation formula for systems of tensor wave equations, arXiv:$1005.4509 \mathrm{v} 1,2010$.

23. E. Stein, Topics in harmonic analysis related to Littlewood-Paley theory, Annals of Mathematical Studies 63 (1970), 145 pages.

24. Q. Wang, Causal geometry of Einstein vacuum space-times, Ph.D. thesis, Princeton University, 2006.

25. 26 (2009), no. 1, 285-328.

26. - Improved breakdown criterion for solution of Einstein vacuum equation in CMC gauge, arXiv:1004.2938v1, 2010.

27. - On Ricci coefficients of null hypersurfaces with time foliation in Einstein vacuum space-time, arXiv:1006.5963v1, 2010.

Department of Mathematics, Princeton University, Princeton NJ 08544

E-mail address: aricks@math.princeton.edu 